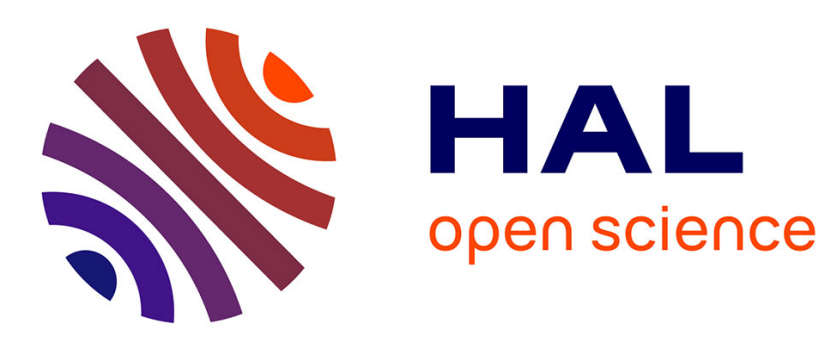

\title{
Generic hyperbolicity of Aubry sets on surfaces
}

Gonzalo Contreras, Alessio Figalli, Ludovic Rifford

\section{To cite this version:}

Gonzalo Contreras, Alessio Figalli, Ludovic Rifford. Generic hyperbolicity of Aubry sets on surfaces. Inventiones Mathematicae, 2015, 200 (1). hal-00935976

\section{HAL Id: hal-00935976 https://hal.science/hal-00935976}

Submitted on 30 Jan 2014

HAL is a multi-disciplinary open access archive for the deposit and dissemination of scientific research documents, whether they are published or not. The documents may come from teaching and research institutions in France or abroad, or from public or private research centers
L'archive ouverte pluridisciplinaire HAL, est destinée au dépôt et à la diffusion de documents scientifiques de niveau recherche, publiés ou non, émanant des établissements d'enseignement et de recherche français ou étrangers, des laboratoires publics ou privés. 


\title{
Generic hyperbolicity of Aubry sets on surfaces
}

\author{
G. Contreras* \\ A. Figalli ${ }^{\dagger}$ \\ L. Rifford ${ }^{\ddagger}$
}

May 21, 2013

\begin{abstract}
Given a Tonelli Hamiltonian of class $C^{2}$ on the cotangent bundle of a compact surface, we show that there is an open dense set of potentials in the $C^{2}$ topology for which the Aubry set is hyperbolic in its energy level.
\end{abstract}

\section{Introduction}

Let $M$ be a smooth compact Riemannian manifold without boundary of dimension $n \geq 2$, and $H: T^{*} M \rightarrow \mathbb{R}$ be a Tonelli Hamiltonian of class $C^{2}$. As shown by Mather [27], one can construct a compact invariant subset of $T^{*} M$ which enjoys several variational properties and has the distinguished feature of being a Lipschitz graph over $M$. This set, called the Aubry set associated to $H$ and denoted by $\tilde{\mathcal{A}}(H)$, captures many important features of the Hamiltonian dynamics.

Fathi [16] established a bridge between the Aubry-Mather theory and the properties of viscosity solutions/subsolutions of the critical Hamilton-Jacobi equation associated with $H$, giving rise to the weak KAM theory. The differentials of critical (viscosity) subsolutions are uniquely determined on the projection of $\tilde{\mathcal{A}}(H)$ onto $M$ (denoted by $\mathcal{A}(H)$ ), and all critical subsolutions are indeed $C^{1,1}$ on the projected Aubry set $\mathcal{A}(H)$. We refer the reader to Section 2.1 below for a precise definition of the Aubry set and more details in weak KAM theory.

A famous open problem concerning the structure of $\tilde{\mathcal{A}}(H)$ is the so-called "Mañé conjecture" [25] which states that, for a generic Hamiltonian, the Aubry set is either a hyperbolic equilibrium or a hyperbolic periodic orbit. In $[19,20]$, the second and third author obtained several results in the direction of proving the validity of the Mañé conjecture. However, all that results heavily rely on the assumption of the existence of a sufficiently smooth critical (sub-)solution. The goal of this paper is to combine some of the techniques developed in [19, 20] with tools from dynamical systems and new regularity estimates for viscosity solutions, to answer in low dimension to an open problem proposed by Herman during the ICM in 1998 [22, Section 6.2, Question 2] (in the context of twist maps on $\mathbb{T}^{1}$, this question was posed by $\mathrm{A}$. Katok, and positively solved by P. Le Calvez [24]):

Is it true that generically the Aubry set is hyperbolic?

As mentioned by Herman at the beginning of [22, Section 6], the subject of the instabilities of Hamiltonian flows and the problem of topological stability "lacks any non-trivial result". Our main theorem solves in the affirmative Herman's problem on surfaces for the $C^{2}$-topology.

\footnotetext{
${ }^{0}$ The second and third authors are supported by the program "Project ANR-07-BLAN-0361, Hamilton-Jacobi et théorie KAM faible". AF is also supported by NSF Grants DMS-0969962 and DSM-1262411. GC is supported by CONACYT Grant 178838. LR is also supported by the ANR project GCM, program "Blanche", project number NT09-504490.

*CIMAT, P.O. Box 402, 36000 Guanajuato GTO, Mexico (gonzalo@cimat.mx)

$\dagger$ Department of Mathematics, The University of Texas at Austin, 2515 Speedway Stop C1200, Austin TX 78712, USA (figalli@math.utexas.edu)

$\ddagger$ Université de Nice-Sophia Antipolis, Labo. J.-A. Dieudonné, UMR CNRS 6621, Parc Valrose, 06108 Nice Cedex 02, France \& Institut Universitaire de France (ludovic.rifford@math.cnrs.fr)
} 
Theorem 1.1. Let $H: T^{*} M \rightarrow \mathbb{R}$ be a Tonelli Hamiltonian of class $C^{2}$, and assume that $\operatorname{dim} M=2$. Then there is an open dense set of potentials $\mathcal{V} \subset C^{2}(M)$ such that, for every $V \in \mathcal{V}$, the Aubry set associated to the Hamiltonian $H+V$ is hyperbolic in its energy level.

The proof of Theorem 1.1 relies on the properties of Green bundles which can be associated with each orbit of the Aubry set. The basic idea is based on the following dichotomy for Green bundles: either they are always transverse, in which case one gets hyperbolicity of the Aubry set; or the Green bundles coincide along a given orbit of the Aubry set, and in this latter case, elaborating on previous works by Arnaud $[4,5]$, we show that the restriction to the projected Aubry set of any critical solution is $C^{2}$ along the projected orbit. This additional regularity property is not enough to apply the techniques which were introduced in $[19,20]$, since there the authors had to require the existence of a critical solution which is $C^{1,1}$ in a neighborhood of the projected orbit and $C^{2}$ along it. In our case, we do not have any regularity property outside the projected orbit, and critical solutions may be merely Lipschitz in any neighborhood of the projected orbit. Still, by some new refined estimates on the regularity of a critical solution near a point where the Green bundles coincide, we are able to exploit the techniques used in $[19,20]$ to conclude the argument and prove our theorem.

Our proof together with the shadowing lemma (see [23]) yields the following closing-type result:

Theorem 1.2. Let $H: T^{*} M \rightarrow \mathbb{R}$ be a Tonelli Hamiltonian of class $C^{2}$, and assume that $\operatorname{dim} M=2$. Then, for every open set $\mathcal{U} \subset T^{*} M$ containing $\tilde{\mathcal{A}}(H)$, and any neighborhood $\mathcal{V}$ of 0 in $C^{2}(M)$, there exist $\theta \in \mathcal{U}$ and $V \in \mathcal{V}$ such that the orbit with respect to the Hamiltonian $H+V$ passing through $\theta$ is periodic and hyperbolic.

We notice that a similar statement could be deduced as a direct consequence of the results in $[25,17,8]$ : more precisely, by [17, Theorem 1.5] and [8, Theorems 1 and 2] the Aubry set is upper-semicontinuous on surfaces, so $[25 \text {, Theorem F }]^{1}$ implies that generically in $C^{\infty}$ topology one can find a periodic orbit close to the Aubry set. However, in contrast with Theorem 1.2 above, this orbit may not be hyperbolic (even if one introduces an additional small perturbation by a potential, see [31]). Therefore, if we work in the $C^{2}$ topology, Theorem 1.1 allows us to say that the Aubry set of $H+V$ is hyperbolic, which in turn implies the hyperbolicity of the sequence of periodic orbits approaching the Aubry set (see Proposition 2.18). All in all, we get the following refinement of $[25$, Theorem $\mathrm{F}]$ in two dimensions and $C^{2}$ topology:

Theorem 1.3. Let $H: T^{*} M \rightarrow \mathbb{R}$ be a Tonelli Hamiltonian of class $C^{2}$, and assume that $\operatorname{dim} M=2$. Then there is a residual set of potentials $\mathcal{G} \subset C^{2}(M)$ such that, for every $V \in \mathcal{G}$, the Lagrangian associated with $H+V$ admits a unique minimizing measure, which is indeed a strong limit of a sequence of probability measures supported on hyperbolic periodic orbits.

The paper is structured as follows. First, we collect several preliminary results which are fundamental for the proof of Theorem 1.1: Section 2.1 is concerned with reminders in weak KAM theory; Section 2.2 contains a result on connecting trajectories; Sections 2.3, 2.4, and 2.5 are devoted to the constructions of Green bundles, paratingent cones, and Arnaud-type results; Section 2.6 contains reminders on hyperbolicity and quasi-hyperbolicity; finally, Sections 2.7 and 2.8 contain material on semiconcave and BV functions, and a lemma from harmonic analysis, which play a major role in the proof of Theorem 1.1. Section 3 is concerned with the proof of Theorem 1.1, which is split into a stability and a density part. Finally, in Section 4 we present some examples of Tonelli Hamiltonians on surfaces of positive genus whose Aubry set is a non-trivial minimal hyperbolic set.

\footnotetext{
${ }^{1}$ Mañé's Theorem [25, Theorem F] asserts that, given a Tonelli Hamiltonian of class $C^{k}$ with $k \geq 2$, there is a residual set of potentials $\mathcal{G} \subset C^{k}(M)$ such that, for every $V \in \mathcal{G}$, the Lagrangian associated with $H+V$ admits a unique minimizing measure, which is indeed a strong limit of a sequence of probability measures supported on periodic orbits.
} 
Acknowledgments: Large part of this work was done while AF was visiting the Mathematics Department at the University of Nice, whose warm hospitality is gratefully acknowledged.

\section{Preliminary results}

\subsection{Reminders in Weak KAM theory}

Recall that a Tonelli Hamiltonian $H: T^{*} M \rightarrow \mathbb{R}$ of class $C^{2}$ is a Hamiltonian satisfying the two following properties:

(H1) Superlinear growth: For every $K \geq 0$ there is a finite constant $C^{*}(K) \in \mathbb{R}$ such that

$$
H(x, p) \geq K\|p\|_{x}+C^{*}(K) \quad \forall(x, p) \in T^{*} M .
$$

(H2) Uniform convexity: For every $(x, p) \in T^{*} M$, the second derivative along the fibers $\frac{\partial^{2} H}{\partial p^{2}}(x, p)$ is positive definite.

The critical value $\mathbf{c}[H] \in \mathbb{R}$ of $H$ may be defined as the infimum of the values $c \in \mathbb{R}$ for which there exists a smooth function $u: M \rightarrow \mathbb{R}$ satisfying

$$
H(x, d u(x)) \leq c \quad \forall x \in M .
$$

A Lipschitz function $u: M \rightarrow \mathbb{R}$ is called a critical subsolution (for $H$ ) if

$$
H(x, d u(x)) \leq \mathbf{c}[H] \quad \text { for a.e. } x \in M .
$$

It can be shown that the set of critical subsolutions, denoted by $\mathcal{S} \mathcal{S}$, is a nonempty compact convex subset of $C^{0}(M ; \mathbb{R})[16,30]$. Fathi and Siconolfi [18] proved that the set of critical subsolutions of class $C^{1}$ (denoted by $\mathcal{S S}^{1}$ ) is nonempty, and their result has been improved later by Bernard [7] who showed the existence of critical subsolutions of class $C^{1,1}$. Also, they proved that the so-called Aubry set can be seen as the nonempty compact subset of $T^{*} M$ defined by

$$
\tilde{\mathcal{A}}(H):=\bigcap_{u \in \mathcal{S S}^{1}}\{(x, d u(x)) \mid x \in M \text { s.t. } H(x, d u(x))=\mathbf{c}[H]\} .
$$

This set is invariant under the Hamiltonian flow, and by Mather's graph property it is a Lipschitz graph over the projected Aubry set defined as

$$
\mathcal{A}(H):=\pi^{*}(\tilde{\mathcal{A}}(H)) \subset M,
$$

where $\pi^{*}: T^{*} M \rightarrow M$ denotes the canonical projection map (see for instance [16, 30]).

The Lagrangian $L: T M \rightarrow \mathbb{R}$ associated with $H$ by Legendre-Fenchel duality is defined by

$$
L(x, v):=\max _{p \in T_{x}^{*} M}\{\langle p, v\rangle-H(x, p)\} \quad \forall(x, v) \in T M .
$$

Thanks to (H1)-(H2), it is easy to see that $L$ is a Tonelli Lagrangian of class $C^{2}$, that is a Lagrangian satisfying both properties of superlinear growth and uniform convexity (see $[9$, 16]). Critical subsolutions have important variational properties, and for instance they can be characterized as follows (see $[16,30])$ :

Proposition 2.1. A function $u: M \rightarrow \mathbb{R}$ is a critical subsolution if and only if

$$
u(\gamma(b))-u(\gamma(a)) \leq \int_{a}^{b} L(\gamma(s), \dot{\gamma}(s)) d s+\mathbf{c}[H](b-a),
$$

for any Lipschitz curve $\gamma:[a, b] \rightarrow M$. 
The (backward) Lax-Oleinik semigroup

$$
\left\{\mathcal{T}_{t}^{-}\right\}_{t \geq 0}: C^{0}(M ; \mathbb{R}) \longrightarrow C^{0}(M ; \mathbb{R})
$$

associated with $L$ is defined as follows: for every $t \geq 0$ and $u \in C^{0}(M ; \mathbb{R})$, the function $\mathcal{T}_{t}^{-} u:=\mathcal{T}_{t}^{-}(u)$ is given by

$$
\mathcal{T}_{t}^{-} u(x):=\inf \left\{u(\gamma(-t))+\int_{-t}^{0} L(\gamma(s), \dot{\gamma}(s)) d s\right\} \quad \forall x \in M,
$$

where the infimum is taken over all Lipschitz curves $\gamma:[-t, 0] \rightarrow M$ such that $\gamma(0)=x$. The set of critical subsolutions $\mathcal{S S}$ is invariant under $\left\{\mathcal{T}_{t}^{-}\right\}_{t \geq 0}$. A critical subsolution $u: M \rightarrow \mathbb{R}$ is called a critical solution or a weak KAM solution if,

$$
\mathcal{T}_{t}^{-} u=u-\mathbf{c}[H] t \quad \forall t \geq 0 .
$$

Critical solutions may be characterized in several ways (see for instance $[16,30]$ ):

Proposition 2.2. Let $u \in C^{0}(M ; \mathbb{R})$. The following properties are equivalent:

(i) $u$ is a critical solution.

(ii) $u \in \mathcal{S S}$ and, for every $x \in M$, there exists a Lipschitz curve $\gamma_{x}:(-\infty, 0] \rightarrow M$ with $\gamma_{x}(0)=x$ such that

$$
u\left(\gamma_{x}(b)\right)-u\left(\gamma_{x}(a)\right)=\int_{a}^{b} L\left(\gamma_{x}(s), \dot{\gamma}_{x}(s)\right) d s+\mathbf{c}[H](b-a) \quad \forall a<b \leq 0 .
$$

(iii) $u \in \mathcal{S S}$ and for every smooth function $\phi: M \rightarrow \mathbb{R}$ with $\phi \leq u$ and all $x \in M$,

$$
\phi(x)=u(x) \quad \Longrightarrow \quad H(x, d \phi(x)) \geq \mathbf{c}[H] .
$$

As shown in [29], critical solutions enjoy some regularity properties. One of them is the fact that critical solutions are semiconcave. Recall that, given an open set $\Omega \subset \mathbb{R}^{n}$, a function $v: \Omega \rightarrow \mathbb{R}^{n}$ is said to be locally semiconcave in $\Omega$ if, for every $x \in \Omega$, there are $C_{x} \geq 0$ and a ball $\mathcal{B}_{x} \subset \Omega$ containing $x$ such that the function $y \mapsto v(y)-C_{x}|y|^{2}$ is concave on $\mathcal{B}_{x}$. A function $v: M \rightarrow \mathbb{R}$ is called locally semiconcave if it is locally semiconcave in charts, that is, if for every $x \in M$ there are an open neighborhood $\mathcal{V}_{x}$ of $x$ and a smooth diffeomorphism $\phi_{x}: \mathcal{V}_{x} \rightarrow \phi_{x}\left(\mathcal{V}_{x}\right) \subset \mathbb{R}^{n}$ such that $v \circ \phi_{x}^{-1}$ is locally semiconcave on $\phi_{x}\left(\mathcal{V}_{x}\right) \subset \mathbb{R}^{n}$. Of course, if the manifold $M$ is compact then the constant $C_{x}$ can be chosen independent of the point, and we say that the function is semiconcave.

Proposition 2.3. Any critical solution is semiconcave on $M$.

Let $u: M \rightarrow \mathbb{R}$ be a critical solution for $H$ and $x \in M$ be fixed. By assertion (ii) in Proposition 2.2 above, there exists a curve $\gamma_{x}:(-\infty, 0] \rightarrow M$ with $\gamma_{x}(0)=x$ satisfying (2.5). Since $u$ is a critical subsolution (see Proposition 2.1), we infer that for every $T>0$ the restriction of $\gamma_{x}$ to $[-T, 0]$ minimizes the quantity

$$
u(\gamma(-T))+\int_{-T}^{0} L(\gamma(s), \dot{\gamma}(s)) d s+\mathbf{c}[H] T,
$$

among Lipschitz curves $\gamma:[-T, 0] \rightarrow M$ such that $\gamma(0)=x$. In particular, $\gamma_{x}$ is the projection of a Hamiltonian trajectory, and whenever $u$ is differentiable at $\gamma_{x}(-T)$, by the first variation formula one gets

$$
d u\left(\gamma_{x}(-T)\right)=\frac{\partial L}{\partial v}\left(\gamma_{x}(-T), \dot{\gamma}_{x}(-T)\right) .
$$


We call limiting differential of $u$ at $x \in M$, and we denote it by $D_{x}^{*} u$, the set of $p \in T_{x}^{*} M$ such that there is a sequence $\left\{x_{k}\right\}_{k}$ of points converging to $x$ such that $u$ is differentiable at $x_{k}$ and $p=\lim _{k \rightarrow \infty} d u\left(x_{k}\right)$. Note that, by the Lipschitz regularity of $u$, the graph of the multivalued mapping $D^{*} u$ is a compact subset of $T^{*} M$. As shown in [29], by the above discussion one can prove that there is a one-to-one correspondence between the limiting differentials and the curves satisfying (2.5):

Proposition 2.4. Let $u: M \rightarrow \mathbb{R}$ be a critical solution and $x \in M$. For every $p \in D_{x}^{*} u$ the curve $\gamma_{x}:(-\infty, 0] \rightarrow M$ defined by

$$
\gamma_{x}(-t):=\pi^{*}\left(\phi_{-t}^{H}(x, p)\right) \quad \forall t \geq 0,
$$

satisfies $\gamma_{x}(0)=x$, (2.5), and

$$
\left(\gamma_{x}(-t), D_{\gamma_{x}(-t)}^{*} u\right)=\left\{\phi_{-t}^{H}(x, p)\right\} \quad \forall t \geq 0 .
$$

In particular $u$ is differentiable at $\gamma_{x}(-t)$ for any $t>0$. Moreover, for every curve $\gamma_{x}$ : $(-\infty, 0] \rightarrow M$ satisfying $\gamma_{x}(0)=x$ and (2.5), there is $p \in D_{x}^{*} u$ such that (2.6) holds.

A curve of the form $\gamma_{x}:(-\infty, 0] \rightarrow M$ satisfying (2.5) is called a semi-calibrated curve. A curve defined on $\mathbb{R}$ satisfying (2.5) for any $a, b \in \mathbb{R}$ is called calibrated. As we said previously, the Aubry set $\tilde{\mathcal{A}}(H)$ is invariant under the Hamiltonian flow, and it is a Lipschitz graph over $\mathcal{A}(H)$. Fathi and Siconolfi [18] proved that, for every point of $\mathcal{A}(H)$, the limiting differential of a critical solution is a singleton there. In particular, since $\tilde{\mathcal{A}}(H)$ is a Lipschitz graph over $\mathcal{A}(H)$, this means that every critical solution $u$ is differentiable on $\mathcal{A}(H)$, its differential is independent of $u$, and $x \mapsto d u(x)$ is Lipschitz on the Aubry set. In addition, for any $(x, p) \in \tilde{\mathcal{A}}(H)$, the curve (2.6) is calibrated. All these facts are summarized in the following:

Proposition 2.5. Let $u: M \rightarrow \mathbb{R}$ be a critical solution and $x \in \mathcal{A}(H)$. Then $u$ is differentiable at $x, d u(x)$ does not depend on $u, D_{x}^{*} u=\{d u(x)\}$, and the calibrated curve $\gamma_{x}: \mathbb{R} \rightarrow M$ defined by

$$
\gamma_{x}(t):=\pi^{*}\left(\phi_{t}^{H}(x, d u(x))\right) \quad \forall t \in \mathbb{R}
$$

satisfies $\gamma_{x}(0)=x$,

$$
u\left(\gamma_{x}(b)\right)-u\left(\gamma_{x}(a)\right)=\int_{a}^{b} L\left(\gamma_{x}(s), \dot{\gamma}_{x}(s)\right) d s+\mathbf{c}[H](b-a) \quad \forall a<b,
$$

$\gamma_{x}(t) \in \mathcal{A}(H)$ for all $t \in \mathbb{R}$, and

$$
\left(\gamma_{x}(t), D_{\gamma_{x}(t)}^{*} u\right)=\left\{\phi_{t}^{H}(x, d u(x))\right\} \quad \forall t \in \mathbb{R} .
$$

Finally, the mapping $\mathcal{A}(H) \ni x \mapsto d u(x)$ is Lipschitz.

We refer the reader to $[19,21]$ for a more detailed introduction to weak KAM theory, to the notes [30] for the proofs of the above results, and to [16] for further details.

\subsection{The Dirichlet problem and the connection of trajectories}

Let $H: \mathbb{R}^{n} \times\left(\mathbb{R}^{n}\right)^{*} \rightarrow \mathbb{R}$ be a Tonelli Hamiltonian of class $C^{2}, V: \mathbb{R}^{n} \rightarrow \mathbb{R}$ a $C^{2}$ function, and denote by $H_{V}$ the Hamiltonian $H+V$. We split $\mathbb{R}^{n}$ as $\mathbb{R} \times \mathbb{R}^{n-1}$ and we define the $(n-1)$-dimensional disks

$$
\Pi_{r}^{\tau}:=\{\tau\} \times B^{n-1}(0, r) \quad \forall \tau \in \mathbb{R}, \forall r>0,
$$


where $B^{n-1}(0, r) \subset \mathbb{R}^{n-1}$ denotes the $(n-1)$-dimensional open ball of radius $r$ centered at the origin. Denoting by $\pi^{*}: \mathbb{R}^{n} \times\left(\mathbb{R}^{n}\right)^{*} \rightarrow \mathbb{R}^{n}$ the projection onto the space variable, we define the following Poincaré-type maps:

Given $\tau>0$ small, $\tau_{1}, \tau_{2} \in[0, \tau]$, and $\left(x^{0}, p^{0}\right) \in \Pi_{1 / 2}^{\tau_{1}} \times \mathbb{R}^{n}$ such that $[-2 \tau, 2 \tau] \ni t \mapsto$ $\pi^{*}\left(\phi_{t}^{H_{V}}\left(x^{0}, p^{0}\right)\right)$ intersects $\Pi_{1}^{\tau_{2}}$ transversally, we define the maps

$$
\mathcal{P}_{\tau_{1}, \tau_{2}}^{*}\left(x^{0}, p^{0}\right):=\phi_{\mathcal{T}_{\tau_{1}, \tau_{2}}^{*}\left(x^{0}, p^{0}\right)}^{H}\left(x^{0}, p^{0}\right), \quad \mathcal{P}_{\tau_{1}, \tau_{2}}\left(x^{0}, p^{0}\right):=\pi^{*}\left(\mathcal{P}_{\tau_{1}, \tau_{2}}^{*}\left(x^{0}, p^{0}\right)\right),
$$

where $\mathcal{T}_{\tau_{1}, \tau_{2}}^{*}\left(x^{0}, p^{0}\right) \in[-2 \tau, 2 \tau]$ is the first time (positive if $\tau_{1}<\tau_{2}$, negative if $\tau_{1}>\tau_{2}$ ) for which $\mathcal{P}_{\tau_{1}, \tau_{2}}\left(x^{0}, p^{0}\right) \in \Pi_{1}^{\tau_{2}}$.

As shown in [19, Lemma 5.1], the following holds:

Lemma 2.6. Let $\bar{u}: B^{n}(0,1) \rightarrow \mathbb{R}$ be a $C^{1,1}$ function such that

$$
\frac{d}{d t}\left(\pi^{*}\left(\phi_{t}^{H}\left(x^{0}, d \bar{u}\left(x^{0}\right)\right)\right)\right)_{\mid t=0} \cdot e_{1} \geq \frac{1}{2} \quad \forall x^{0} \in \Pi_{1}^{0} .
$$

Then there exists $\bar{\tau}>0$ small such that the following properties are satisfied:

(i) For every $\tau \in(0,5 \bar{\tau}]$, the Poincaré time mapping $\mathcal{T}_{0, \tau}^{d \bar{u}}: \Pi_{1 / 2}^{0} \rightarrow \mathbb{R}$ defined by

$$
\mathcal{T}_{0, \tau}^{d \bar{u}}\left(x_{0}\right):=\mathcal{T}_{0, \tau}^{*}\left(x^{0}, d \bar{u}\left(x^{0}\right)\right) \quad \forall x^{0} \in \Pi_{1 / 2}^{0},
$$

is well-defined and of class $C^{k-1}$;

(ii) for every $\tau \in(0,5 \bar{\tau}]$, the Poincaré mapping $\mathcal{P}_{0, \tau}^{d \bar{u}}: \Pi_{1 / 2}^{0} \rightarrow \Pi_{1}^{\tau}$ defined by

$$
\mathcal{P}_{0, \tau}^{d \bar{u}}:=\mathcal{P}_{0, \tau}\left(x^{0}, d \bar{u}\left(x^{0}\right)\right) \quad \forall x^{0} \in \Pi_{1 / 2}^{0},
$$

is 2-Lipschitz;

(iii) the following inclusion holds for every $\tau \in(0,5 \bar{\tau}]$ :

$$
\left\{\pi^{*}\left(\phi_{t}^{H}\left(x^{0}, d \bar{u}\left(x^{0}\right)\right)\right) \mid x^{0} \in \Pi_{3 / 8}^{0}, t \in\left[0, \mathcal{T}_{0, \tau}\left(x^{0}\right)\right]\right\} \subset[0, \tau] \times B^{n-1}(0,1 / 2) ;
$$

(iv) the viscosity solution $\bar{u}_{0}$ to the Dirichlet problem

$$
\left\{\begin{array}{l}
H\left(z, d \bar{u}_{0}(z)\right)=0 \quad \text { in }[0,5 \bar{\tau}] \times B^{n-1}(0,1 / 2), \\
\bar{u}_{0}=\bar{u} \text { on } \Pi_{1}^{0},
\end{array}\right.
$$

is of class $C^{1,1}$.

We now define the cylinder

$$
\mathcal{C}\left(\left(x^{0}, p^{0}\right) ; t ; r\right):=\left\{\pi^{*}\left(\phi_{t}^{H}\left(x^{0}, p^{0}\right)\right)+(0, \hat{y})|s \in[0, t],| \hat{y} \mid<r\right\},
$$

and the action

$$
\begin{array}{r}
\mathbb{A}_{V}\left(\left(x^{0}, p^{0}\right) ; \tau\right):=\int_{0}^{\tau} L_{V}\left(\pi^{*}\left(\phi_{t}^{H_{V}}\left(x^{0}, p^{0}\right)\right), \frac{d}{d t}\left(\pi^{*}\left(\phi_{t}^{H_{V}}\left(x^{0}, p^{0}\right)\right)\right)\right) d t \\
=\int_{0}^{\tau} L\left(\pi^{*}\left(\phi_{t}^{H_{V}}\left(x^{0}, p^{0}\right)\right), \frac{d}{d t}\left(\pi^{*}\left(\phi_{t}^{H_{V}}\left(x^{0}, p^{0}\right)\right)\right)\right) \\
-V\left(\pi^{*}\left(\phi_{t}^{H_{V}}\left(x^{0}, p^{0}\right)\right)\right) d t
\end{array}
$$

where $\phi_{t}^{H_{V}}$ denotes the Hamiltonian flows associated to $H_{V}$. By the results in [19], the following holds: 
Proposition 2.7. Let $u: B^{n}(0,1) \rightarrow \mathbb{R}$ be a viscosity solution of $H(x, d u(x))=\mathbf{c}[H]$ and assume that

$$
\begin{gathered}
\frac{d}{d t}\left(\pi^{*}\left(\phi_{t}^{H}\left(x^{0}, p^{0}\right)\right)\right)_{\mid t=0} \cdot e_{1} \geq \frac{1}{2} \quad \forall x^{0} \in \Pi_{1}^{0}, \forall p^{0} \in D_{x^{0}}^{*} u, \\
\left\langle p_{0}, \dot{\gamma}_{0, p_{0}}(0)\right\rangle \geq-c_{0} \quad \forall p_{0} \in D_{0}^{*} u, \quad \text { where } \quad \gamma_{0, p_{0}}(t):=\pi^{*}\left(\phi_{t}^{H}\left(0, p_{0}\right)\right)
\end{gathered}
$$

for some small positive constant $c_{0}$. Then, for any $\bar{\tau}>0$ sufficiently small there are $\bar{\delta}, \bar{r}, \bar{\epsilon} \in$ $(0,1 / 4)$ and $K>0$ such that the following property holds: For any $r \in(0, \bar{r}), \hat{\epsilon} \in(0, \bar{\epsilon})$, $x^{0} \in \Pi_{1}^{0}, x^{f} \in \Pi_{1}^{\bar{\tau}}, p^{0} \in D_{x^{0}}^{*} u, p^{f} \in D_{x^{f}}^{*} u$, and $\sigma \in \mathbb{R}$ satisfying

$$
\left|x^{0}\right|<\bar{\delta}
$$

and

$$
\left|\left(x^{f}, p^{f}\right)-\mathcal{P}_{0, \bar{\tau}}^{*}\left(x^{0}, p^{0}\right)\right|<r \hat{\epsilon}, \quad|\sigma|<r^{2} \hat{\epsilon}
$$

there exist a time $T^{f}>0$ and a potential $V: \mathbb{R}^{n} \rightarrow \mathbb{R}$ of class $C^{2}$ such that:

(i) $\operatorname{Supp}(V) \subset \mathcal{C}\left(\left(x^{0}, p^{0}\right) ; \mathcal{T}_{0, \bar{\tau}}^{*}\left(x^{0}, p^{0}\right) ; r\right)$;

(ii) $\|V\|_{C^{2}}<K \hat{\epsilon}$;

(iii) $\left|T^{f}-\mathcal{T}_{0, \bar{\tau}}^{*}\left(x^{0}, p^{0}\right)\right|<K r \hat{\epsilon} ;$

(iv) $\phi_{T^{f}}^{H_{V}}\left(x^{0}, p^{0}\right)=\left(x^{f}, p^{f}\right)$;

(v) $\mathbb{A}_{V}\left(\left(x^{0}, p^{0}\right) ; T^{f}\right)=\mathbb{A}\left(\left(x^{0}, p^{0}\right) ; \mathcal{T}_{0, \bar{\tau}}^{*}\left(x^{0}, p^{0}\right)\right)+\left\langle d u\left(\mathcal{P}_{0, \bar{\tau}}\left(x^{0}, p^{0}\right)\right), x^{f}-\mathcal{P}_{0, \bar{\tau}}\left(x^{0}, p^{0}\right)\right\rangle+\sigma$.

Proof. First of all, it follows by (2.13) and Lemma 2.6(ii) that, provided $\bar{\tau}$ is sufficiently small (the smallness being independent of $r$ and $\hat{\epsilon}$ ),

$$
\left|\mathcal{P}_{\bar{\tau}, \bar{\tau} / 2}^{*}\left(x^{f}, p^{f}\right)-\mathcal{P}_{0, \bar{\tau} / 2}^{*}\left(x^{0}, p^{0}\right)\right|<2 r \hat{\epsilon} .
$$

Hence, we first apply [19, Proposition 3.1] on $[0, \bar{\tau} / 2]$ to connect $\left(x^{0}, p^{0}\right)$ to $\mathcal{P}_{\bar{\tau}, \bar{\tau} / 2}^{*}\left(x^{f}, p^{f}\right)$ in a time $T_{1}^{f} \sim \bar{\tau} / 2$ with a "default" of action bounded by $K r^{2} \hat{\epsilon}^{2}$. Then, thanks to (2.11), assuming $c_{0}$ and $\bar{\epsilon}$ sufficiently small we can apply [19, Proposition 4.1] on $[\bar{\tau} / 2, \bar{\tau}]$ to "compensate" the default of action so that (v) above holds. Moreover it is easily seen that also all the other properties are satisfied. We leave the details to the reader.

\subsection{Green bundles and reduced Green bundles}

Let us endow the cotangent bundle $T^{*} M$ with its standard symplectic structure $\omega$, and denote by $V_{\theta}:=\operatorname{ker}\left(d_{\theta} \pi^{*}\right)$ the vertical space in $T_{\theta}\left(T^{*} M\right)$ at any $\theta \in T^{*} M$ (recall that $\pi^{*}: T^{*} M \rightarrow M$ denotes the canonical projection). A subspace $E \subset T_{\theta}\left(T^{*} M\right)$ is called Lagrangian if it is a $n$ dimensional vector subspace where the symplectic bilinear form $\omega_{\theta}: T_{\theta}\left(T^{*} M\right) \times T_{\theta}\left(T^{*} M\right) \rightarrow \mathbb{R}$ vanishes. As an example, vertical spaces are Lagrangian. If we fix a symplectic set of local coordinates, we can identify $T_{\theta}\left(T^{*} M\right)$ with $T_{x} M \times T_{x}^{*} M$ and $V_{\theta}$ with $\{0\} \times T_{x}^{*} M$. Then, any $n$-dimensional vector subspace $E \subset T_{\theta}\left(T^{*} M\right)$ which is transversal to $V_{\theta}$ (i.e. $E \cap V_{\theta}=\{0\}$ ) can be written as the graph of some linear map $S: T_{x} M \rightarrow T_{x}^{*} M$, and it can be checked that $E$ is Lagrangian if and only if $S$ is represented by a symmetric matrix.

Given a Hamiltonian $H: T^{*} M \rightarrow \mathbb{R}$ of class $C^{2}$, the Hamiltonian vector field $X_{H}$ on $T^{*} M$ is defined by $\omega_{\theta}\left(X_{H}(\theta), \cdot\right)=-d_{\theta} H$ for any $\theta \in T^{*} M$. In a symplectic set of local coordinates, the Hamiltonian equations (i.e., the equations satisfied by any solution of the ODE 
$\left.(\dot{x}, \dot{p})=X_{H}((x, p))\right)$ are given by $\dot{x}=\frac{\partial H}{\partial p}, \dot{p}=-\frac{\partial H}{\partial x}$. Finally, we recall that the Hamiltonian flow $\phi_{t}^{H}$ of $X_{H}$ preserves the symplectic form $\omega$. In particular, the image of a Lagrangian space $E \subset T_{\theta}\left(T^{*} M\right)$ by $D_{\theta} \phi_{t}^{H}$ is Lagrangian in $T_{\phi_{t}^{H}(\theta}\left(T^{*} M\right)$. We refer the reader to $[1,10]$ for more details about the notions of symplectic geometry introduced above.

We recall now the construction and properties of Green bundles and reduced Green bundles along orbits of the Hamiltonian flow without conjugate points. We refer the reader to $[4,6,12]$ for further details and historical accounts. For every $\theta \in T^{*} M$ and every $t \in \mathbb{R}$, we define the Lagrangian subspace $G_{\theta}^{t} \subset T_{\theta}\left(T^{*} M\right)$ as the pushforward of the vertical distribution at $\phi_{-t}^{H}(\theta)$ by $\phi_{t}^{H}$, that is

$$
G_{\theta}^{t}:=\left(\phi_{t}^{H}\right)_{*}\left(V_{\phi_{-t}^{H}(\theta)}\right)=D_{\phi_{-t}^{H}(\theta)} \phi_{t}^{H}\left(V_{\phi_{-t}^{H}(\theta)}\right) \quad \forall \theta \in T^{*} M .
$$

The orbit of $\theta \in T^{*} M$ is said to be without conjugate points if for any $t, t^{\prime} \in \mathbb{R}$,

$$
t \neq t^{\prime} \Longrightarrow\left[D_{\phi_{t}^{H}(\theta)} \phi_{t^{\prime}-t}^{H}\left(V_{\phi_{t}^{H}(\theta)}\right)\right] \cap V_{\phi_{t^{\prime}}^{H}(\theta)}=\{0\} .
$$

We denote by $\mathcal{D}$ the set of $\theta \in T^{*} M$ whose orbit has no conjugate point, and we assume that $\mathcal{D}$ is nonempty. Given $\theta \in \mathcal{D} \subset T^{*} M$, and fix a symplectic set of local coordinates around $\theta=(x, p)$. Then, for every $t \in \mathbb{R} \backslash\{0\}$, the Lagrangian subspace $G_{\theta}^{t}$ is transverse to the vertical subspace $V_{\theta}$ in $T_{\theta}\left(T^{*} M\right) \simeq T_{x} M \times T_{x}^{*} M$. Hence, there is a linear operator $K_{\theta}^{t}: T_{x} M \rightarrow T_{x}^{*} M$ such that

$$
G_{\theta}^{t}=\left\{\left(h, K_{\theta}^{t} h\right) \in T_{x} M \times T_{x}^{*} M \mid h \in T_{x} M\right\} .
$$

Since $G_{\theta}^{t}$ is Lagrangian, the linear operator $K_{\theta}^{t}$ can be represented by a symmetric matrix in our symplectic set of local coordinates. There is a natural partial order for the Lagrangian subspaces which are transverse to the vertical, which simply corresponds to the usual order for symmetric operators. Later on, given two Lagrangian subspaces $E, E^{\prime} \in T_{\theta}\left(T^{*} M\right)$ which are transverse to $V_{\theta}$, we shall write $E \prec E^{\prime}$ (resp. $E \preceq E^{\prime}$ ) if the corresponding symmetric operators $K, K^{\prime}$ are such that $K^{\prime}-K$ is positive definite (resp. nonnegative definite). The following property is a consequence of the uniform convexity of $H$ in the fibers (see [4, Proposition 3.7] and [12, Proposition 1.4]):

Proposition 2.8. Let $\theta \in \mathcal{D}$. The following properties hold:

(i) For every $t^{\prime}>t>0, G_{\theta}^{t^{\prime}} \prec G_{\theta}^{t}$.

(ii) For every $t^{\prime}<t<0, G_{\theta}^{t} \prec G_{\theta}^{t^{\prime}}$.

(iii) For every $t<0<t^{\prime}, G_{\theta}^{t} \prec G_{\theta}^{t^{\prime}}$.

As a consequence, for every $\theta \in \mathcal{D}$, the sequence of Lagrangian subspaces $(0,+\infty) \ni t \mapsto G_{\theta}^{t}$ (resp. $\left.(0,+\infty) \ni t \mapsto G_{\theta}^{-t}\right)$ is decreasing (resp. increasing) and bounded from below by $G_{\theta}^{-1}$ (resp. bounded from above by $G_{\theta}^{1}$ ). Hence, both limits as $t \rightarrow \pm \infty$ exist, which leads to the following definition:

Definition 2.9. For every $\theta \in \mathcal{D}$, we define the positive and negative Green bundles at $\theta$ as

$$
G_{\theta}^{+}:=\lim _{t \rightarrow+\infty} G_{\theta}^{t} \quad \text { and } \quad G_{\theta}^{-}:=\lim _{t \rightarrow-\infty} G_{\theta}^{t} .
$$

We shall keep in mind that the positive Green bundle $G_{\theta}^{+}$depends on the behavior of the Hamiltonian flow along the orbit of $\theta$ for large negative times, while the negative Green bundle $G_{\theta}^{-}$depends on what happens for large positive times. By construction, we also have the following result (see [4, Corollaire 3.8 and Proposition 3.9] and [12, Proposition 1.4 (d)]): 
Proposition 2.10. Let $\theta \in \mathcal{D}$. The following properties hold:

(i) $G_{\theta}^{-} \preceq G_{\theta}^{+}$.

(ii) $D_{\theta} \phi_{t}^{H}\left(G_{\theta}^{-}\right)=G_{\phi_{t}^{H}(\theta)}^{-}$and $D_{\theta} \phi_{t}^{H}\left(G_{\theta}^{+}\right)=G_{\phi_{t}^{H}(\theta)}^{+}$for all $t \in \mathbb{R}$.

Moreover, the function $\theta \mapsto G_{\theta}^{+}$is upper-semicontinuous on $\mathcal{D}$, and $\theta \mapsto G_{\theta}^{-}$is lower-semicontinuous on $\mathcal{D}$. Thus, if $G_{\theta}^{+}=G_{\theta}^{-}$for some $\theta \in \mathcal{D}$ then both of them are continuous at $\theta$.

The following result, which first appeared in [12], plays a major role in recent works by Arnaud [4, 5, 6] (see [4, Proposition 3.12], [6, Proposition 1], and [12, Proposition 1.11]):

Proposition 2.11. Let $\theta \in \mathcal{D}$ and $\psi \in T_{\theta}\left(T^{*} M\right)$. Then the following properties hold:

(i) $\psi \notin G_{\theta}^{-} \Longrightarrow \lim _{t \rightarrow+\infty}\left\|D_{\theta}\left(\pi^{*} \circ \phi_{t}^{H}\right)(\psi)\right\|=+\infty$.

(ii) $\psi \notin G_{\theta}^{+} \Longrightarrow \lim _{t \rightarrow-\infty}\left\|D_{\theta}\left(\pi^{*} \circ \phi_{t}^{H}\right)(\psi)\right\|=+\infty$.

For every $\theta=(x, p) \in T^{*} M$, denote by $\Sigma_{\theta} \subset T^{*} M$ the energy level

$$
\Sigma_{\theta}:=\left\{\theta^{\prime}=\left(x^{\prime}, p^{\prime}\right) \in T^{*} M \mid H\left(x^{\prime}, p^{\prime}\right)=H(x, p)\right\} .
$$

From the previous result one easily gets the following conclusion (see [4, Exemple 2 page 17] and $[12$, Corollary 1.12]):

Proposition 2.12. Let $\theta \in \mathcal{D}$ be such that $X_{H}(\theta) \neq 0$. Then

$$
X_{H}(\theta) \in G_{\theta}^{-} \cap G_{\theta}^{+} \quad \text { and } \quad G_{\theta}^{-} \cup G_{\theta}^{+} \subset T_{\theta} \Sigma_{\theta} .
$$

Let $\Sigma \subset T^{*} M$ be a regular energy level of $H$, that is an energy level satisfying $\frac{\partial H}{\partial p}(x, p) \neq 0$ for every $\theta=(x, p) \in \Sigma$. By superlinear growth (H1) and uniform convexity (H2) of $H$, the hypersurface $\Sigma$ is compact and, for every $\theta=(x, p) \in \Sigma$, the fiber $\Sigma \cap T_{x}^{*} M$ is the boundary of a uniformly convex set in $T_{x}^{*} M$. For every $\theta \in \Sigma$ we define the subspace $N_{\theta} \subset T_{\theta} \Sigma$ by

$$
N_{\theta}:=\left\{\psi \in T_{\theta} \Sigma \mid\left\langle D_{\theta} \pi^{*}(\psi), D_{\theta} \pi^{*}\left(X_{H}(\theta)\right)\right\rangle_{\pi^{*}(\theta)}=0\right\},
$$

where $\langle\cdot, \cdot\rangle$ denotes the Riemannian metric on $M$. By construction, we have

$$
T_{\theta} \Sigma=N_{\theta} \oplus \mathbb{R} X_{H}(\theta) \quad \forall \theta \in \Sigma .
$$

For every $\theta \in \mathcal{D} \cap \Sigma$, we define the reduced Green bundles $\hat{G}_{\theta}^{-}$and $\hat{G}_{\theta}^{+}$as

$$
\hat{G}_{\theta}^{-}:=G_{\theta}^{-} \cap N_{\theta} \quad \text { and } \quad \hat{G}_{\theta}^{+}:=G_{\theta}^{+} \cap N_{\theta} .
$$

As shown in [4], the reduced Green bundles can be seen as the Green bundles associated with a specific symplectic bundle over the orbit of $\theta$; they satisfy the same properties of the Green bundles, in particular Proposition 2.10. If $M$ has dimension two, then, for every $\theta \in \mathcal{D} \cap \Sigma$, the reduced Green bundles $\hat{G}_{\theta}^{+}$and $\hat{G}_{\theta}^{-}$should be seen as lines in the plane $N_{\theta} \simeq T_{\theta} \Sigma / \mathbb{R} X_{H}(\theta)$. Finally we observe that, since $G_{\theta}^{+}$depends on the behavior of the Hamiltonian flow near $\phi_{t}^{H}(\theta)$ for large negative times, its construction can be performed as soon as the orbit of $\theta \in T^{*} M$ has no conjugate points in negative time. In particular, this can be done for any semi-calibrated curve (see Proposition 2.4). 


\subsection{Paratingent cones and Green bundles}

The present section is inspired by ideas and techniques developed by Arnaud in [4, 5], and by the last two authors in [20]. Let $S \subset \mathbb{R}^{k}$ be a compact set which has the origin as a cluster point. The paratingent cone to $S$ at 0 is the cone defined as

$$
C_{0}(S):=\left\{\lambda \lim _{i \rightarrow \infty} \frac{x_{i}-y_{i}}{\left|x_{i}-y_{i}\right|} \mid \lambda \in \mathbb{R}, \lim _{i \rightarrow \infty} x_{i}=\lim _{i \rightarrow \infty} y_{i}=0, x_{i} \in S, y_{i} \in S, x_{i} \neq y_{i} \forall i\right\},
$$

and the paratingent space of $S$ at 0 is the vector space generated by $C_{0}(S)$ :

$$
\Pi_{0}(S):=\operatorname{Span}\left\{C_{0}(S)\right\} .
$$

As shown in [20, Lemma 3.3], the set $S$ is contained locally in the graph of a function from $\Pi:=\Pi_{0}(S)$ onto its orthogonal complement $\Pi^{\perp}$. Let $d$ be the dimension of $\Pi$, denote by $\operatorname{Proj}_{\Pi}$ the orthogonal projection onto the space $\Pi$ in $\mathbb{R}^{k}$, and set $\mathcal{H}_{S}:=\operatorname{Proj}_{\Pi}(S)$. Finally, for any $r, \nu>0$ we define the cylinder

$$
C(r, \nu):=\left\{(h, v) \in \Pi \times \Pi^{\perp}|| h|<r,| v \mid<\nu\right\},
$$

where $|\cdot|$ denotes the Euclidean norm. Also, we set $B_{r}:=B(0, r)$.

Lemma 2.13. There exist $r_{S}>0$ and a Lipschitz function $\Psi_{S}: \Pi \cap \bar{B}_{r_{S}} \rightarrow \Pi^{\perp}$ such that the following properties hold:

(i) $\left.S \cap C\left(r_{S}, r_{S}\right) \subset \operatorname{graph}\left(\Psi_{S}\right)\right|_{B_{r_{S}}}:=\left\{h+\Psi_{S}(h) \mid h \in \Pi \cap B_{r_{S}}\right\}$;

(ii) $h+\Psi_{S}(h)$ belongs to $S \cap C\left(r_{S}, r_{S}\right)$ for every $h \in \mathcal{H}_{S} \cap B_{r_{S}}$;

(iii) For any $r \in\left(0, r_{S}\right)$, let $\ell(r)>0$ denote the Lipschitz constant of $\Psi_{S}$ on $\Pi \cap B_{r}$. Then $\lim _{r \downarrow 0} \ell(r)=0$.

In particular $\Psi_{S}(0)=0, \Psi_{S}$ is $C^{1}$ at 0 , and $\nabla \Psi_{S}(0)=0$.

By Proposition 2.5, through each point $\theta=(x, p)$ of the Aubry set $\tilde{\mathcal{A}}(H)$ passes a calibrated curve (defined by (2.8)) which corresponds to the projection of its orbit under the Hamiltonian flow, and whose restriction to any subinterval is always minimizing the action between its endpoints. Being minimizing, such a curve has necessarily no conjugate points, hence $\theta \in \mathcal{D}$. We also observe that, since the Aubry set is invariant under the Hamiltonian flow,

$$
D_{\theta} \phi_{t}^{H}\left(C_{\theta}(\tilde{\mathcal{A}}(H))\right)=C_{\phi_{t}^{H}(\theta)}(\tilde{\mathcal{A}}(H)) \quad \forall t \in \mathbb{R}
$$

and $X_{H}(\theta)$ belongs to the paratingent cone to $\tilde{\mathcal{A}}(H)$ at $\theta$, that is

$$
X_{H}(\theta) \in C_{\theta}(\tilde{\mathcal{A}}(H)) \subset T_{\theta} \Sigma_{H} \quad \forall \theta \in \tilde{\mathcal{A}}(H),
$$

where $\Sigma_{H}:=\{H=\mathbf{c}[H]\}$. Given $\theta=(x, p) \in \tilde{\mathcal{A}}(H)$ with $X_{H}(\theta) \neq 0$, we define the reduced paratingent cone to the Aubry set as

$$
\hat{C}_{\theta}:=C_{\theta}(\tilde{\mathcal{A}}(H)) \cap N_{\theta},
$$

where $N_{\theta}$ has been defined in Section 2.3. If $M$ has dimension two, $\hat{C}_{\theta}$ is a collection of lines in the plane $N_{\theta}$. All those lines can be compared with other lines in this plane. The following proposition is a variant of Arnaud's results (compare with [4, Proposition 3.11], [4, Proposition 3.16 (3)], [6, Theorem 9]), and it follows from the Lipschitz graph property of the Aubry set. 
Proposition 2.14. Assume that $\operatorname{dim} M=2$ and that $\theta \in \tilde{\mathcal{A}}(H)$ is not an equilibrium of $X_{H}$. Then

$$
\hat{G}_{\theta}^{-} \preceq \hat{C}_{\theta} \preceq \hat{G}_{\theta}^{+} .
$$

Proof. Since $\tilde{\mathcal{A}}(H)$ is a Lipschitz graph, its paratingent cones cannot intersect the vertical bundle, hence taking $\epsilon>0$ small enough yields

$$
G_{\theta}^{-\epsilon} \prec C_{\theta}:=C_{\theta}(\tilde{\mathcal{A}}(H)) \prec G_{\theta}^{\epsilon} .
$$

For every $t \neq 0$ the Lagrangian space $G_{\theta}^{t}$ is transverse to $V_{\theta}$, it does not contain $X_{H}(\theta)$, and it is contained in $T_{\theta} \Sigma_{H}$. Hence, its intersection with $N_{\theta}$ is a line in the plane $N_{\theta}$. The inequality (2.17) means that the intersection of $C_{\theta}$ with $N_{\theta}$ is a collection of vector lines which are squeezed between the lines $G_{\theta}^{-\epsilon} \cap N_{\theta}$ and $G_{\theta}^{\epsilon} \cap N_{\theta}$. Therefore, to prove the result, it is sufficient to show that no line $G_{\theta}^{t} \cap N_{\theta}$ with $t \in \mathbb{R} \backslash[-\epsilon, \epsilon]$ is contained in $C_{\theta} \cap N_{\theta}$. Argue by contradiction and assume that there is $\bar{t}>\epsilon$ (the other case is left to the reader) such that

$$
G_{\theta}^{-\bar{t}} \cap N_{\theta} \subset C_{\theta} \cap N_{\theta} .
$$

By (2.14)-(2.15), this means that $V_{\phi_{t}^{H}(\theta)}$ and $D_{\theta} \phi_{\bar{t}}^{H}\left(C_{\theta}\right)=C_{\phi_{\bar{t}}^{H}(\theta)}$ do intersect, which contradicts the Lipschitz graph property of the Aubry set.

As an application of Proposition 2.14 and Lemma 2.13, we deduce that if $\operatorname{dim} M=2$ and the positive and negative Green bundles coincide for some $\theta=(x, p) \in \tilde{\mathcal{A}}(H)$ with $X_{H}(\theta) \neq 0$, then the Aubry set is locally contained in the graph of a Lipschitz 1-form which is $C^{1}$ at $x$. It will be convenient to extend the 1-form along a piece of projected orbit of the Aubry set.

Corollary 2.15. Assume that $\operatorname{dim} M=2$ and that $\theta=(x, p) \in \tilde{\mathcal{A}}(H)$ with $X_{H}(\theta) \neq 0$ satisfies

$$
G_{\theta}^{-}=G_{\theta}^{+} \text {. }
$$

Assume moreover that $\theta$ is not on a periodic orbit and let $\gamma(t):=\pi^{*}\left(\phi_{t}^{H}(\theta)\right)$ for any $t \in \mathbb{R}$. Then, for every $T>0$ there are an open neighborhood $\mathcal{V}$ of $\gamma([-T, T])$ in $M$ and a function $f: \mathcal{V} \rightarrow \mathbb{R}$ of class $C^{1,1}$ which is $C^{2}$ along $\gamma([-T, T])$ such that

$$
\tilde{\mathcal{A}}(H) \cap T^{*} \mathcal{V} \subset \operatorname{Graph}(d f),
$$

and for every $t \in[-T, T], G_{\phi_{t}^{H}(\theta)}^{-}=G_{\phi_{t}^{H}(\theta)}^{+}$is the graph of $D_{\gamma(t)}^{2} f$ (in a symplectic set of local coordinates in $\left.T^{*} \mathcal{V}\right)$.

Proof. By Proposition 2.12 and (2.16), if the two Green bundles coincide, the paratingent cone $C_{\theta}:=C_{\theta}(\tilde{\mathcal{A}}(H))$ is a Lagrangian plane which is transverse to the vertical subspace $V_{\theta}$. Then, working in a symplectic set of local coordinates, by Lemma 2.13 we deduce that are an open neighborhood $\mathcal{U}$ of $x$, and a Lipschitz 1 -form $\Psi$ on $\mathcal{U}$ which is $C^{1}$ at $x$, such that

$$
\tilde{\mathcal{A}}(H) \cap T^{*} \mathcal{U} \subset \operatorname{Graph}(\Psi)=G_{\theta}^{-}=G_{\theta}^{+},
$$

and the Lagrangian plane $C_{\theta}$ coincides with the graph of $d_{x} \Psi$. Since $\frac{\partial H}{\partial p}(\theta) \neq 0$ (because $X_{H}(\theta) \neq 0$ and the Aubry set is a Lipschitz graph), the set of $\theta^{\prime} \in T^{*} M$ with $H\left(\theta^{\prime}\right)=\mathbf{c}[H]$ is locally (in a neighborhood of $\theta$ ) a submanifold of dimension 3 of class $C^{2}$. Then up to compose $\Psi$ with a retraction $r$ of class at least $C^{1}$ onto the set $\{H=\mathbf{c}[H]\}$, we may assume that $\Psi$ is a Lipschitz 1-form satisfying

$$
H(\Psi(x))=\mathbf{c}[H] \quad \forall x \in \mathcal{U} .
$$

Let $S \subset \mathcal{U}$ be a local section (that is, a smooth curve) which is transverse to $\gamma$ at $x$. By the properties of $\Psi$, the map $\Phi:[-2 T, 2 T] \times S \rightarrow M$ defined by

$$
\Phi(t, y):=\pi^{*}\left(\phi_{t}^{H}(\Psi(y))\right) \quad \forall t \in[-2 T, 2 T], \forall y \in S,
$$


is Lipschitz, and it is $C^{1}$ along the segment $[-T, T] \times\{0\}$. Moreover, since $C_{\theta}=G_{\theta}^{-}=G_{\theta}^{+}$, the differential of $\Phi$ is invertible at $(t, 0)$ for every $t \in[-T, T]$. Therefore, by the Clarke Lipschitz Inverse Function Theorem (see [11, Theorem 5.1.1]), $\Phi$ admits a Lipschitz inverse $\Phi^{-1}=(\tau, \epsilon): \mathcal{V} \rightarrow[-2 T, 2 T] \times S$ in a simply connected neighborhood $\mathcal{V}$ of $\gamma([-T, T])$ (remember that $\gamma$ is not periodic) which is $C^{1}$ along $\gamma([-T, T])$. By construction, the 1 -form $\alpha$ on $\mathcal{V}$ defined by

$$
\alpha(x):=\pi^{*}\left(\phi_{t}^{H}(\tau(x), \epsilon(x))\right) \quad \forall x \in \mathcal{V},
$$

is a closed Lipschitz 1-form which is $C^{1}$ along the curve $\gamma([-T, T])$. By the Poincaré lemma, we get a function satisfying the conclusions of Corollary 2.15 .

We notice that an alternative way to perform the above construction is to approach $\Psi$ by a sequence of 1-form of class $C^{1}$, to construct a sequence of functions of class $C^{2}$ by the method of characteristics (see [16]) and to get the $C^{1,1}$ function $f$ by taking the limit. Such an approach can be found in $[15]$.

\subsection{Hessians and positive Green bundles}

As shown by Alexandrov (see for instance $[14,33]$ ), locally semiconcave functions are two times differentiable almost everywhere.

Theorem 2.16. Let $U$ be an open subset of $\mathbb{R}^{n}$ and $u: U \rightarrow \mathbb{R}$ be a function which is locally semiconcave on $U$. Then, for a.e. $x \in U, u$ is differentiable at $x$ and there exists a symmetric operator $A(x): \mathbb{R}^{n} \rightarrow \mathbb{R}^{n}$ such that the following property is satisfied:

$$
\lim _{t \downarrow 0} \frac{u(x+t v)-u(x)-t d u(x) \cdot v-\frac{t^{2}}{2}\langle A(x) \cdot v, v\rangle}{t^{2}}=0 \quad \forall v \in \mathbb{R}^{n} .
$$

Moreover, $x \mapsto d u(x)$ is differentiable a.e. in $U$ (that is for a.e. $x \in U$, any section of $z \mapsto D_{z}^{*} u$ is differentiable at $x)$, and its differential is given by $A(x)$.

We infer that, if $u: M \rightarrow \mathbb{R}$ is semiconcave, then for almost every $x \in M, u$ is differentiable at $x, D_{x}^{*} u$ is a singleton, $d u$ is differentiable at $x$ and the graph of its differential is a Lagrangian subspace $D_{x}^{2} u \subset T_{(x, d u(x))}\left(T^{*} M\right)$. Notice that if $u: M \rightarrow \mathbb{R}$ is a critical solution, then by Proposition 2.4 regularity properties of $u$ propagate in negative time. That is, for every $x \in M$ such that $u$ is two times differentiable at $x$, the function $u$ is two times differentiable along the semi-calibrated curve $\gamma_{x}:(-\infty, 0] \rightarrow M$ given by (2.6). Moreover we have

$$
D_{(x, d u(x))} \phi_{-t}^{H}\left(D_{x}^{2} u\right)=D_{\gamma_{x}(-t)}^{2} u \quad \forall t \geq 0 .
$$

Recall that for every $\theta=(x, p) \in D^{*} u$, the Hamiltonian trajectory starting at $\theta$ at time zero has no conjugate points in negative times (see Proposition 2.4), which allows us to construct $G_{\theta}^{+}$at any such points. Then, proceeding as in the proof of Proposition 2.14 (replacing (2.15) by (2.18)) we obtain the following one-sided estimate (notice that, since $D_{x}^{2} u$ is a Lagrangian subspace, the assumption on the dimension of $M$ could be dropped, see the proof of Proposition 2.14 and [4, Proposition 3.11]):

Proposition 2.17. Assume that $\operatorname{dim} M=2$, let $u: M \rightarrow \mathbb{R}$ be a critical solution, and let $x \in M$ be such that $d u(x)$ and $D^{2} u(x)$ exist and $X_{H}(x, d u(x)) \neq 0$. Then

$$
D_{x}^{2} u \preceq G_{(x, d u(x))}^{+} .
$$

Later on, in the proof of Theorem 1.1, the above result together with the upper semicontinuity of the positive Green bundle will allow us to obtain a local bound from above for $D^{2} u$ in a neighborhood of a given point of the projected Aubry set (see (3.13)). 


\subsection{Reminders on hyperbolicity}

Recall that $\phi_{t}^{H}$ denotes the Hamiltonian flow in $T^{*} M$. A compact $\phi_{t}^{H}$-invariant set $\Lambda \subset T^{*} M$ is called hyperbolic in its energy level $\Sigma$ with respect to the Hamiltonian flow if the following properties are satisfied:

(h1) At each point $\theta \in \Lambda$, the tangent space $T_{\theta} \Sigma$ is the direct sum of three subspaces $E_{\theta}^{s}, E_{\theta}^{u}$, and $E_{\theta}^{0}=\mathbb{R} X_{H}(\theta)$.

(h2) At each point $\theta \in \Lambda$, we have $D_{\theta} \phi_{t}^{H}\left(E_{\theta}^{s}\right)=E_{\phi_{t}^{H}(\theta)}^{s}$ and $D_{\theta} \phi_{t}^{H}\left(E_{\theta}^{u}\right)=E_{\phi_{t}^{H}(\theta)}^{u}$, for any $t \in \mathbb{R}$.

(h3) There are a Riemannian metric in an open neighborhood of $\Lambda$, and constants $C \geq 1$ and $\mu>0$ such that, for each $\theta \in \Lambda, \psi^{s} \in E_{\theta}^{s}$, and $\psi^{u} \in E_{\theta}^{u}$, we have

$$
\begin{aligned}
\| D_{\theta} \phi_{t}^{H}\left(\psi^{s}\right) & \leq C e^{-\mu t}\left\|\psi^{s}\right\| \\
\left\|D_{\theta} \phi_{-t}^{H}\left(\psi^{u}\right)\right\| & \leq C e^{-\mu t}\left\|\psi^{u}\right\|
\end{aligned}
$$

for all $t>0$.

Note that, as a consequence of hyperbolicity, the splitting $T_{\theta} \Sigma=E_{\theta}^{s} \oplus E_{\theta}^{u} \oplus E_{\theta}^{0}$ defined for $\theta \in \Lambda$ is continuous. Let us extend it into a continuous (not necessarily invariant) splitting $T_{\theta} \Sigma_{\theta}=E_{\theta}^{s} \oplus E_{\theta}^{u} \oplus E_{\theta}^{0}$ with $E_{\theta}^{0}=\mathbb{R} X_{H}(\theta)$ defined for all $\theta$ in an open neighborhood $\mathcal{V}$ of $\Lambda$. Then, for every $\theta \in \mathcal{V}$ and any $\rho \in(0,1)$, we define the family of horizontal and vertical cones $\left\{H_{\theta}^{\rho}\right\}$ and $\left\{V_{\theta}^{\rho}\right\}$ as

$$
\begin{aligned}
H_{\theta}^{\rho} & :=\left\{\xi+\eta \mid \xi \in E_{\theta}^{u}, \eta \in E_{\theta}^{s},\|\eta\| \leq \rho\|\xi\|\right\}, \\
V_{\theta}^{\rho} & :=\left\{\xi+\eta \mid \xi \in E_{\theta}^{u}, \eta \in E_{\theta}^{s},\|\xi\| \leq \rho\|\eta\|\right\} .
\end{aligned}
$$

By (h2)-(h3), for every $\theta \in \Lambda, \rho \in(0,1)$, and $t>0$, we have

$$
\begin{gathered}
D_{\theta} \phi_{t}^{H}\left(H_{\theta}^{\rho}\right) \subset H_{\phi_{t}^{H}(\theta)}^{e^{-2 \mu t} C^{2} \rho}, \quad D_{\theta} \phi_{-t}^{H}\left(V_{\theta}^{\gamma}\right) \subset V_{\phi_{-t}^{H}(\theta)}^{e^{-2 \mu t} C^{2} \rho}, \\
\left\|D_{\theta} \phi_{t}^{H}(\psi)\right\| \geq \frac{e^{\mu t}}{C^{\prime}(1+\rho)}\|\psi\| \quad \forall \psi \in H_{\theta}^{\rho}, \\
\left\|D_{\theta} \phi_{-t}^{H}(\psi)\right\| \geq \frac{e^{\mu t}}{C^{\prime}(1+\rho)}\|\psi\| \quad \forall \psi \in V_{\theta}^{\rho} .
\end{gathered}
$$

Hence, by continuity and compactness, we can find $T>0, \mu^{\prime}>0$, an open neighborhood $\mathcal{V}^{\prime} \subset \mathcal{V}$, and continuous disjoint cones $\mathbb{S}_{\theta}, \mathbb{U}_{\theta} \subset E_{\theta}^{s} \oplus E_{\theta}^{u}$ containing $E_{\theta}^{s}, E_{\theta}^{u}$ respectively, such that, for every $\theta \in \mathcal{V}^{\prime}$,

$$
D_{\theta} \phi_{T}^{H}\left(\mathbb{U}_{\theta}\right) \subset \operatorname{Int}\left(\mathbb{U}_{\phi_{T}^{H}(\theta)}\right), \quad D_{\theta} \phi_{-T}^{H}\left(\mathbb{S}_{\theta}\right) \subset \operatorname{Int}\left(\mathbb{S}_{\phi_{-T}^{H}(\theta)}\right),
$$

and

$$
\begin{array}{cc}
\left\|D_{\theta} \phi_{T}^{H}(\psi)\right\| \geq e^{\mu^{\prime} T}\|\psi\| \quad \forall \psi \in \mathbb{U}_{\theta}, \\
\left\|D_{\theta} \phi_{-T}^{H}(\psi)\right\| \geq e^{\mu^{\prime} T}\|\psi\| \quad \forall \psi \in \mathbb{S}_{\theta} .
\end{array}
$$

This shows that any $\phi_{t}^{H}$-invariant compact set sufficiently close to $\Lambda$ will satisfy the Alekseev cone criterion, which provides an alternative more handy characterization for hyperbolicity (see [23]). This criterion is also robust under perturbation of the dynamics, and allows us to obtain that following: 
Proposition 2.18. Let $\Lambda \subset T^{*} M$ be a compact $\phi_{t}^{H}$-invariant set which is hyperbolic in its energy level with respect to the Hamiltonian flow. Then there exists an open neighborhood $\mathcal{V}$ of 0 in $C^{2}(M)$ and an open neighborhood $\mathcal{O}$ of $\Lambda$ such that, for every potential $V \in \mathcal{V}$, any compact set $\Lambda^{\prime} \subset \mathcal{O}$ which is $\phi_{t}^{H+V}$-invariant is hyperbolic in its energy level with respect to $\phi_{t}^{H+V}$.

The above result will be useful to show the stability part (that is openness) of Theorem 1.1. As shown in $[12, \S 3]$, a way to obtain hyperbolicity is to show quasi-hyperbolicity properties. Let $B$ be a compact metric space and $\pi: E \rightarrow B$ a vector bundle equipped with a continuous norm $|\cdot|_{p}$ on each fiber $\pi^{-1}(p)$. Let $\Psi$ be a continuous $\mathbb{R}$-action $\Psi_{t}: \mathbb{R} \rightarrow \operatorname{Isom}(E)$ such that $\Psi_{s+t}=\Psi_{s} \circ \Psi_{t}$. We say that $\Psi$ is quasi-hyperbolic if

$$
\sup _{t \in \mathbb{R}}\left\{\left|\Psi_{t}(\xi)\right|\right\}=+\infty \quad \forall \xi \in E \backslash\{0\} .
$$

The following result holds (see [12, Theorem 0.2], and compare with [5, §3] and [6, Theorem $2])$ :

Proposition 2.19. Assume that any point in $B$ is non-wandering and that $\Psi$ is quasi-hyperbolic. Then $\Psi$ is hyperbolic.

In the proof of Theorem 1.1, the above result allows us to obtain the hyperbolicity of the Aubry set almost for free in the case when the Green bundles are always transverse. Such an approach is nowadays classical.

\subsection{Some properties of semiconcave and BV functions}

\subsubsection{Derivatives of semiconcave functions}

Let $v: \mathbb{R}^{n} \rightarrow \mathbb{R}$ be a semiconcave function, i.e., $v$ can be written as the sum of a concave function and a smooth function. Since second distributional derivatives of convex functions are nonnegative Radon measures (see [14, §6.3]), the Radon-Nikodým Theorem [2, Theorem 1.28] allows us to write $D^{2} v$ as the sum of an absolutely continuous matrix-valued measure and a singular matrix-valued measure:

$$
D^{2} v=\nabla^{2} v d x+D_{\mathcal{S}}^{2} v,
$$

where $\nabla^{2} v \in L_{l o c}^{1}$ is the pointwise Hessian of $v$ (which exists almost everywhere by Alexandrov's Theorem), and $D_{\mathcal{S}}^{2} v$ is a singular measure (with respect to the Lebesgue measure). Also, by semiconcavity we have that $D^{2} v$ is locally bounded from above (as a measure): for any $R>0$ there exists a constant $C_{R}>0$ such that

$$
\int_{E}\left\langle D^{2} v \cdot e, e\right\rangle \leq C_{R}|E| \quad \forall E \subset B^{n}(0, R) \text { Borel, } \forall e \in \mathbb{R}^{n} \text { with }|e|=1 .
$$

In particular, choosing $E$ of measure zero we get

$$
\int_{E}\left\langle D_{\mathcal{S}}^{2} v \cdot e, e\right\rangle \leq 0 \quad \forall E \subset \mathbb{R}^{n} \text { Borel with }|E|=0, \forall e \in \mathbb{R}^{n} \text { with }|e|=1 .
$$

Hence, since the measure $D_{\mathcal{S}}^{2} v$ is singular with respect to the Lebesgue measure, by the arbitrariness of $E$ we deduce that $\left\langle D_{\mathcal{S}}^{2} v \cdot e, e\right\rangle$ is a negative singular measure for any vector $e \in \mathbb{R}^{n}$.

Since the distributional derivative of $\nabla v$ is equal to the measure $D^{2} v$, by definition $\nabla v$ : $\mathbb{R}^{n} \rightarrow \mathbb{R}^{n}$ is a function of bounded variation (see $[2, \S 3]$ ). Given $x^{\prime} \in \mathbb{R}^{n-1}$, let us consider the function $w_{x^{\prime}}: \mathbb{R} \rightarrow \mathbb{R}^{n}$ defined by

$$
w_{x^{\prime}}(s):=\nabla v\left(x^{\prime}, s\right) \quad \text { for a.e. } s \in \mathbb{R} .
$$


Note that, since $v$ is differentiable almost everywhere, by Fubini's theorem the function $w_{x^{\prime}}$ is defined for almost every $x^{\prime} \in \mathbb{R}^{n-1}$. It is well-known that the functions $w_{x^{\prime}}$ are of bounded variation on $\mathbb{R}$ for almost every $x^{\prime} \in \mathbb{R}^{n-1}$ as well (see [2, Theorem 3.103] and the subsequent discussion), so their distributional derivative on $\mathbb{R}$ is a measure which can be decomposed as the sum of an absolutely continuous and a singular part:

$$
D w_{x^{\prime}}=\nabla w_{x^{\prime}} d s+D_{\mathcal{S}} w_{x^{\prime}},
$$

where here $D$ is the distributional derivative on $\mathbb{R}, \nabla w_{x^{\prime}} \in L_{l o c}^{1}$ is the pointwise derivative of $w_{x^{\prime}}$ which exists almost everywhere [2, Theorem 3.28(c)], and $D_{\mathcal{S}} w_{x^{\prime}}$ is singular with respect to the one-dimensional Lebesgue measure. Also, the fundamental theorem of calculus holds between every couple of points where $\nabla v$ exists [2, Theorem 3.28]:

$$
\nabla v\left(x^{\prime}, s_{2}\right)-\nabla v\left(x^{\prime}, s_{1}\right)=w_{x^{\prime}}\left(s_{2}\right)-w_{x^{\prime}}\left(s_{1}\right)=\int_{s_{1}}^{s_{2}} \nabla w_{x^{\prime}}(s) d s+\int_{s_{1}}^{s_{2}} d\left(D_{\mathcal{S}} w_{x^{\prime}}\right)(s),
$$

for every $s_{1}<s_{2}$ in $\mathbb{R}$.

Let us recall that, given a vector-valued measure $\mu$, one denotes by $|\mu|$ its total variation, which is defined as

$$
|\mu|(E):=\sup \left\{\sum_{h=0}^{\infty}\left|\mu\left(E_{h}\right)\right|: E_{h} \text { disjoint Borel sets s.t. } E=\bigcup_{h=0}^{\infty} E_{h}\right\} \quad \forall E \text { Borel. }
$$

It is easy to check that, with this definition, $\left|\int_{E} d \mu\right| \leq \int_{E} d|\mu|$. Hence, it follows from (2.21) that

$$
\left|\nabla v\left(x^{\prime}, s_{2}\right)-\nabla v\left(x^{\prime}, s_{1}\right)\right| \leq \int_{s_{1}}^{s_{2}}\left|\nabla w_{x^{\prime}}(s)\right| d s+\int_{s_{1}}^{s_{2}} d\left|D_{\mathcal{S}} w_{x^{\prime}}\right|(s) .
$$

Finally, we recall that the derivative of $w_{x^{\prime}}$ is related to $D^{2} v$ : if we define the family of lines $\ell_{x^{\prime}}:=\left\{\left(x^{\prime}, s\right): s \in \mathbb{R}\right\}$, it follows from [2, Theorem 3.107] that

$$
\int_{E}\left\langle D^{2} v \cdot e_{n}, e\right\rangle=\int_{\mathbb{R}^{n-1}} d x^{\prime} \int_{E \cap \ell_{x^{\prime}}} D w_{x^{\prime}} \cdot e \quad \forall E \subset \mathbb{R}^{n} \text { Borel, } \forall e \in \mathbb{R}^{n} .
$$

This has the following useful consequences: since the measures

$$
\left\langle D_{\mathcal{S}}^{2} v \cdot e_{n}, e\right\rangle-D_{\mathcal{S}} w_{x^{\prime}} \cdot e \quad \text { and } \quad\left(\left\langle\nabla^{2} v \cdot e_{n}, e\right\rangle-\nabla_{\mathcal{S}} w_{x^{\prime}} \cdot e\right) d x
$$

are mutually singular, we deduce that $(2.22)$ holds with $\nabla^{2} v \cdot e_{n}$ and $\nabla w_{x^{\prime}}$ (resp., with $D_{\mathcal{S}}^{2} v \cdot e_{n}$ and $\left.D_{\mathcal{S}} w_{x^{\prime}}\right)$ in place of $D^{2} v \cdot e_{n}$ and $D w_{x^{\prime}}$. Hence

$$
\begin{gathered}
\nabla w_{x^{\prime}}(s)=\nabla^{2} v\left(x^{\prime}, s\right) \cdot e_{n} \quad \text { for a.e. }\left(x^{\prime}, s\right) \in \mathbb{R}^{n}, \\
\int_{E}\left\langle D_{\mathcal{S}}^{2} v \cdot e_{n}, e\right\rangle=\int_{\mathbb{R}^{n-1}} d x^{\prime} \int_{E \cap \ell_{x^{\prime}}} D_{\mathcal{S}} w_{x^{\prime}} \cdot e \quad \forall E \subset \mathbb{R}^{n} \text { Borel, } \forall e \in \mathbb{R}^{n} .
\end{gathered}
$$

(see also [2, Theorem 3.107]). In particular, (2.19) and (2.24) imply that

$$
D_{\mathcal{S}} w_{x^{\prime}} \cdot e_{n} \text { is a negative measure for a.e. } x^{\prime} \in \mathbb{R}^{n-1} .
$$

\subsubsection{The case of a critical solution}

We now gather some extra properties when $v=u$ solves the Hamilton-Jacobi equation. Let us assume that $u: B_{2}=B^{n}(0,2) \rightarrow \mathbb{R}$ is a semiconcave function satisfying

$$
H(x, \nabla u(x))=\mathbf{c}[H] \quad \text { for a.e. } x \in B_{2} .
$$


Then $w:=\nabla u$ is a function of bounded variation, and since semiconcave functions are locally Lipschitz, $w$ is locally bounded inside $B_{2}$. Let us consider the family of bounded Borel functions $a_{h}: B_{1}=B^{n}(0,1) \rightarrow \mathbb{R}, h \in(0,1 / 2)$, defined as

$$
a_{h}(x):=\int_{0}^{1} \frac{\partial H}{\partial x_{n}}\left(x+\tau h e_{n}, \tau \nabla u\left(x+h e_{n}\right)+(1-\tau) \nabla u(x)\right) d \tau
$$

and the family of bounded Borel vector fields $\xi_{h}: B_{1} \rightarrow \mathbb{R}^{n}, h \in(0,1 / 2)$, given by

$$
\xi_{h}(x):=\int_{0}^{1} \frac{\partial H}{\partial p}\left(x+\tau h e_{n}, \tau \nabla u\left(x+h e_{n}\right)+(1-\tau) \nabla u(x)\right) d \tau .
$$

Let us recall that, since $w \in B V_{l o c}\left(B_{2}\right)$, the following bound holds:

$$
\int_{B_{r}} \frac{\left|w\left(x+h e_{n}\right)-w(x)\right|}{h} d x \leq \int_{B_{r+h}}\left|D w \cdot e_{n}\right|(d x)<\infty \quad \forall r \in(0,1), h \in(0,1 / 2)
$$

(for smooth functions the above estimate follows from the fundamental theorem of calculus, and for the general case one argues by approximation using [2, Theorem 3.9]). Hence the measures

$$
\mu_{h}:=\frac{w\left(x+h e_{n}\right)-w(x)}{h} d x
$$

satisfy

$$
\int_{B_{1}}\left|\mu_{h}\right|(d x) \leq C
$$

which implies that, up to a subsequence, $\mu_{h}$ (resp. $\left.\left|\mu_{h}\right|\right)$ converge weakly* to a finite measure $\mu$ (resp. $\nu$ ) as $h \rightarrow 0$. Also, there exists $\bar{a}: B_{1} \rightarrow[0,+\infty)$ bounded such that $\left|a_{h}\right| \rightarrow^{*} \bar{a}$ in $L^{\infty}\left(B_{1}\right)$.

It is easy to show that $\mu=D w \cdot e_{n}=D^{2} u \cdot e_{n}$. Furthermore, it follows from [2, Example $1.63]$ and (2.27) that

$$
\nu\left(B_{r}\right) \leq \liminf _{h \rightarrow 0}\left|\mu_{k}\right|\left(B_{r}\right) \leq \int_{\bar{B}_{r}}\left|D w \cdot e_{n}\right|=|\mu|\left(\bar{B}_{r}\right) \quad \forall r \in(0,1),
$$

so letting $r \nearrow 1$ we obtain $\nu\left(B_{1}\right) \leq|\mu|\left(B_{1}\right)$. This information combined with the bound $|\mu| \leq \nu$ (see [2, Proposition 1.62(b)]) implies that $|\mu|=\nu$, thus

$$
\left|\mu_{h}\right| \rightarrow^{*}|\mu| \text {. }
$$

We now exploit the fact that $u$ solves the Hamilton-Jacobi equation (2.26). Since

$$
\begin{aligned}
0= & H\left(x+h e_{n}, \nabla u\left(x+h e_{n}\right)\right)-H(x, \nabla u(x)) \\
= & h \int_{0}^{1} \frac{\partial H}{\partial x_{n}}\left(x+\tau h e_{n}, \tau \nabla u\left(x+h e_{n}\right)+(1-\tau) \nabla u(x)\right) d \tau \\
& +\left(\int_{0}^{1} \frac{\partial H}{\partial p}\left(x+\tau h e_{n}, \tau \nabla u\left(x+h e_{n}\right)+(1-\tau) \nabla u(x)\right) d \tau\right) \cdot\left(\nabla u\left(x+h e_{n}\right)-\nabla u(x)\right) \\
= & h a_{h}(x)+\xi_{h}(x) \cdot\left(w\left(x+h e_{n}\right)-w(x)\right),
\end{aligned}
$$

we have

$$
a_{h}+\xi_{h} \cdot \mu_{h} \equiv 0 .
$$

Let $\Omega \subset B_{1}$ be an open set and assume that there exist $h_{0}>0$ and a continuous vector field $\Xi: \Omega \rightarrow \mathbb{R}^{n}$ such that

$$
\left|\Xi(x)-\xi_{h}(x)\right| \leq \frac{1}{2}|\Xi(x)| \quad \forall x \in \Omega, \forall h \in\left(0, h_{0}\right) .
$$


Then

$$
0=a_{h}+\xi_{h} \cdot \mu_{h}=a_{h}+\left(\xi_{h}-\Xi\right) \cdot \mu_{h}+\Xi \cdot \mu_{h},
$$

so that, thanks to [2, Proposition 1.62(b)] and (2.28)-(2.29), letting $h \rightarrow 0$ we obtain

$$
|\Xi \cdot \mu| \leq \liminf _{h \rightarrow 0}\left|\Xi \cdot \mu_{h}\right| \leq \liminf _{h \rightarrow 0} \frac{1}{2}|\Xi|\left|\mu_{h}\right|+\left|a_{h}\right|=\frac{1}{2}|\Xi||\mu|+\bar{a} \quad \text { inside } \Omega,
$$

where $|\Xi \cdot \mu|$ denotes the total-variation of the measure $\Xi \cdot \mu$ (and analogously for $\mu_{h}$ ), and $|\Xi|$ denotes the continuous function $x \mapsto|\Xi(x)|$.

We now recall that, as observed above, the measure $\mu$ coincides with the measure $D^{2} u \cdot e_{n}$, hence

$$
\left|\left\langle D^{2} u \cdot e_{n}, \Xi\right\rangle\right| \leq \frac{1}{2}|\Xi|\left|D^{2} u \cdot e_{n}\right|+\bar{a} \quad \text { inside } \Omega .
$$

In particular, if we restrict this inequality to the singular part of $D^{2} u$, since $\bar{a}$ is a bounded function we get

$$
\left|\left\langle D_{\mathcal{S}}^{2} u \cdot e_{n}, \Xi\right\rangle\right| \leq \frac{1}{2}|\Xi|\left|D_{\mathcal{S}}^{2} u \cdot e_{n}\right| \quad \text { inside } \Omega,
$$

which by $(2.22)$ can be written as a superposition of the measures $D w_{x^{\prime}}$ :

$$
\left|\Xi \cdot D_{\mathcal{S}} w_{x^{\prime}}\right| \leq \frac{1}{2}|\Xi|\left|D_{\mathcal{S}} w_{x^{\prime}}\right| \quad \text { inside } \Omega \text {, for a.e. } x^{\prime} .
$$

Using the polar decomposition theorem [2, Corollary 1.29], we can write $D_{\mathcal{S}} w_{x^{\prime}}=\theta\left|D_{\mathcal{S}} w_{x^{\prime}}\right|$, where $\theta: B_{1} \rightarrow \mathbb{S}^{n-1}$ is a $\left|D_{\mathcal{S}} w_{x^{\prime}}\right| \otimes d x^{\prime}$-measurable function. Hence the above equation can be rewritten as

$$
|\Xi \cdot \theta| \leq \frac{1}{2}|\Xi| \quad \text { inside } \Omega,\left|D_{\mathcal{S}} w_{x^{\prime}}\right| \otimes d x^{\prime} \text {-a.e.. }
$$

This information is particularly useful when $n=2$ and $\Xi$ never vanishes: indeed, assuming for instance that $\Xi \equiv e_{1}$, then (2.30) implies that

$$
|\theta| \leq 2\left|\theta \cdot e_{2}\right| \quad \text { inside } \Omega,\left|D_{\mathcal{S}} w_{x^{\prime}}\right| \otimes d x^{\prime} \text {-a.e. }
$$

from which we get

$$
\left|D_{\mathcal{S}} w_{x^{\prime}}\right| \leq 2\left|D_{\mathcal{S}} w_{x^{\prime}} \cdot e_{2}\right| \quad \text { inside } \Omega \text {, for a.e. } x^{\prime} .
$$

This means that $\left|D_{\mathcal{S}}^{2} u \cdot e_{2}\right|$ is controlled by $\left|\left\langle D_{\mathcal{S}}^{2} u \cdot e_{2}, e_{2}\right\rangle\right|$, or equivalently, since $D_{\mathcal{S}}^{2} u \cdot e_{2}$ is a vector-valued measure of components $\left\langle D_{\mathcal{S}}^{2} u \cdot e_{2}, e_{1}\right\rangle$ and $\left\langle D_{\mathcal{S}}^{2} u \cdot e_{2}, e_{2}\right\rangle$, the measure $\left|\left\langle D_{\mathcal{S}}^{2} u \cdot e_{2}, e_{1}\right\rangle\right|$ is controlled by $\left|\left\langle D_{\mathcal{S}}^{2} u \cdot e_{2}, e_{2}\right\rangle\right|$. Hence, the size of the pure second derivatives in the $e_{2}$ direction controls the size of the mixed second derivatives in $e_{1}, e_{2}$ in the region where the Hessian is singular (that is, roughly speaking, where $\nabla u$ has a jump).

\subsection{A lemma from harmonic analysis}

In this section we recall a classical result from harmonic analysis (see [32]), and we show its simple proof for the convenience of the reader. We denote by $|A|$ the Lebesgue measure of a set $A \subset \mathbb{R}^{n}$.

Lemma 2.20. Let $f \in L^{1}\left(\mathbb{R}^{n}\right)$, and define the maximal function

$$
M f(x):=\sup _{x \in \bar{B}, B \text { open ball }}\left\{\frac{1}{|B|} \int_{B}|f(y)| d y\right\} \quad \forall x \in \mathbb{R}^{n} .
$$

There exists a dimensional constant $C_{n}>0$ such that

$$
\left|\left\{x \in \mathbb{R}^{n}: M f(x)>\delta\right\}\right| \leq \frac{C_{n}}{\delta}\|f\|_{L^{1}\left(\mathbb{R}^{n}\right)} \quad \forall \delta>0 .
$$


Proof. Let $K \subset\{M f>\delta\}$ be any compact subset. By the definition of $M f$, for any $x \in K$ there exists an open ball $B_{x}$ such that

$$
x \in \bar{B}_{x}, \quad\left|B_{x}\right| \leq \frac{1}{\delta} \int_{B_{x}}|f(y)| d y .
$$

Let $\rho B$ denote the dilation of a ball $B$ by a factor $\rho>0$ with respect to its center. Since $x \in \bar{B}_{x} \subset 2 B_{x}$, the family of open balls $\left\{2 B_{x}\right\}_{x \in K}$ covers $K$. So, by compactness we can find a finite collection of these balls which still covers $K$, and by Vitali's Lemma [14, §1.5.1, Theorem $1]$ we can select a disjoint subcollection $\left\{2 B_{x_{1}}, \ldots, 2 B_{x_{m}}\right\}$ such that $K \subset \cup_{j=1}^{m} 10 B_{x_{j}}$. Hence

$$
|K| \leq 10^{n} \sum_{j=1}^{m}\left|B_{x_{j}}\right| \leq \frac{10^{n}}{\delta} \sum_{j=1}^{m} \int_{B_{r_{j}}\left(x_{j}\right)}|f(y)| d y \leq \frac{10^{n}}{\delta}\|f\|_{L^{1}\left(\mathbb{R}^{n}\right)},
$$

and the result follows by the arbitrariness of $K$.

\section{Proof of Theorem 1.1}

Let $H: T^{*} M \rightarrow \mathbb{R}$ be a Tonelli Hamiltonian of class $C^{2}$, and denote by $L: T M \rightarrow \mathbb{R}$ its associated Lagrangian. We want to show that the set of potentials $V \in C^{2}(M)$ such that the Aubry set $\tilde{\mathcal{A}}(H+V)$ is hyperbolic contains an open dense set. Hence we need to prove a stability result (the openness) and a density result.

We proceed as follows: First, in Section 3.1 we show that if the Aubry set $\tilde{\mathcal{A}}(H)$ is minimal and hyperbolic, then all Aubry sets $\tilde{\mathcal{A}}(H+V)$ associated with potentials $V \in C^{2}(M)$ which are sufficiently small in $C^{2}$ topology are hyperbolic. Then, in Section 3.2 we show that the set of potentials $V \in C^{2}(M)$ such that the Aubry set of $H+V$ is minimal and hyperbolic is dense. We recall that a nonempty compact $\phi_{t}^{H}$-invariant set $\Lambda \subset T^{*} M$ is called minimal if any orbit of $\phi_{t}^{H}$ contained in $\Lambda$ is dense inside $\Lambda$. By Zorn's Lemma, any nonempty compact $\phi_{t}^{H}$-invariant set contains a minimal subset.

\subsection{The stability part}

Recall that the Peierls barrier is the function $h: M \times M \rightarrow \mathbb{R}$ defined as

$$
h(x, y):=\liminf _{t \rightarrow+\infty}\left\{h_{t}(x, y)+\mathbf{c}[H] t\right\} \quad \forall x, y \in M,
$$

where

$$
h_{t}(x, y):=\inf \int_{0}^{t} L(\gamma(s), \dot{\gamma}(s)) d s
$$

and the infimum is taken over all Lipschitz curves $\gamma:[0, t] \rightarrow M$ such that $\gamma(0)=x$ and $\gamma(t)=y$ (we refer the reader to $[16,18,30]$ for further details). By construction $h$ is Lipschitz on $M \times M$ (see for instance [16, Corollary 5.3.3]) and any critical subsolution $u$ satisfies

$$
u(y)-u(x) \leq h(x, y) \quad \forall x, y \in M
$$

(this fact follows easily from Proposition 2.1). Moreover, it can be checked that (see [16, Proposition 5.3.8], [18, 30])

$$
\mathcal{A}(H)=\{x \in M \mid h(x, x)=0\} .
$$

Following Mather [27], the function $\delta_{M}: M \times M \rightarrow \mathbb{R}$ given by

$$
\delta_{M}(x, y):=h(x, y)+h(y, x) \quad \forall x, y \in M
$$

is a semi-distance (sometimes called the Mather semi-distance). 
Lemma 3.1. Assume that $\tilde{\mathcal{A}}(H)$ is minimal. Then $H$ admits a unique weak KAM solution (up to a constant) and $\delta_{M}(x, y)=0$ for any $x, y \in \mathcal{A}(H)$.

Proof. Let $u_{1}, u_{2}: M \rightarrow \mathbb{R}$ be two weak KAM solutions. Since their differentials coincide along any orbit of the Aubry set (see Proposition 2.5) and in addition all the orbits are dense in $\mathcal{A}(H)$, there is a constant $a \in \mathbb{R}$ such that $u_{1}-u_{2}=a$ on $\mathcal{A}(H)$. By Fathi's comparison theorem (see [16, Theorem 8.5.5]), we infer that $u_{1}$ and $u_{2}$ differ by a constant on the whole $M$. The second assertion follows from the fact that the pointed functions $\{h(z, \cdot)\}_{z \in M}$ are weak KAM solutions (see [16, Theorem 5.3.6] or [18, Proposition 4.1]) and from the equality (using (3.4))

$$
\delta_{M}(x, y)=(h(x, y)-h(x, x))-(h(y, y)-h(y, x)) \quad \forall x, y \in \mathcal{A}(H) .
$$

As shown in [13, Theorem C], by the uniqueness of weak KAM solutions (or equivalently the uniqueness of static classes) one obtains the upper-semicontinuity of the mapping $V \mapsto$ $\tilde{\mathcal{A}}(H+V)$ (compare with [8, corollary 5]), from which the stability of the hyperbolicity of Aubry sets follows:

Lemma 3.2. Assume that $\tilde{\mathcal{A}}(H)$ is minimal and hyperbolic. Then there is an open neighborhood $\mathcal{V}$ of 0 in $C^{2}(M)$ such that, for every $V \in \mathcal{V}, \tilde{\mathcal{A}}(H+V)$ is hyperbolic.

Proof. We first show that, since $H$ admits a unique weak KAM solution (which follows from the previous lemma), the mapping $V \in C^{2}(M) \mapsto \tilde{\mathcal{A}}(H+V) \subset T^{*} M$ is upper semicontinuous with respect to the Hausdorff topology, that is, for every open set $\mathcal{O} \in T^{*} M$ containing $\tilde{\mathcal{A}}(H)$ there is an open neighborhood $\mathcal{V}$ of 0 in $C^{2}(M)$ such that, for every $V \in C^{2}(M)$,

$$
V \in \mathcal{V} \Longrightarrow \tilde{\mathcal{A}}(H+V) \subset \mathcal{O} \text {. }
$$

Without loss of generality, up to adding a constant to $H$ we can assume that $\mathbf{c}[H]=0$.

We argue by contradiction and assume that there are an open neighborhood $\mathcal{O}$ of $\tilde{\mathcal{A}}(H)$, a sequence of potentials $\left\{V_{k}\right\}_{k}$ which tends to zero in the $C^{2}$ topology, and a sequence $\left\{\theta_{k}\right\}_{k} \subset$ $T^{*} M$ satisfying $\theta_{k} \in \tilde{\mathcal{A}}\left(H+V_{k}\right) \backslash \mathcal{O}$ for all $k$. For every $k$, we pick a critical solution $u_{k}$ for the Hamiltonian $H+V_{k}$, and we define the calibrated curves $\gamma_{k}(t):=\pi^{*}\left(\phi_{t}^{H+V_{k}}\left(\theta_{k}\right)\right)$. Taking subsequences if necessary, we may assume that $\left\{u_{k}\right\}_{k}$ converge to a weak KAM solution $u$ for $H$, and $\left\{\gamma_{k}\right\}_{k}$ converge to a calibrated (with respect to $u$ ) curve $\gamma: \mathbb{R} \rightarrow M$ with $\gamma(0) \notin \mathcal{A}(H)$, that is,

$$
u(\gamma(b))-u(\gamma(a))=\int_{a}^{b} L(\gamma(s), \dot{\gamma}(s)) d s=h_{b-a}(\gamma(a), \gamma(b)) \quad \forall a<b .
$$

It can be shown that $\omega$-limit and $\alpha$-limit sets of any calibrated curves are contained in the Aubry set (see [30, Proposition 4.1]). Hence, there is a sequence $\left\{T_{l}\right\}_{l} \uparrow+\infty$ such that $\gamma\left(T_{l}\right)$ and $\gamma\left(-T_{l}\right)$ tend to $\mathcal{A}(H)$ as $l$ tends to $+\infty$. Let us denote by $d$ a Riemannian distance on $M$, and by $K$ a Lipschitz constant for $h$.

Given $\eta>0$ we choose $l$ large enough and $\alpha_{l}, \beta_{l} \in \mathcal{A}(H)$ such that

$$
d\left(\gamma\left(-T_{l}\right), \alpha_{l}\right)+d\left(\gamma\left(T_{l}\right), \beta_{l}\right)<\eta .
$$

Set $x:=\gamma(0)$. Then, using the definition of $h(3.1)$, the fact that $h_{t+s}(x, y) \leq h_{t}(x, z)+h_{s}(z, y)$, 
(3.5), and that $\delta_{M}\left(\alpha_{l}, \beta_{l}\right)=0$ (which follows from Lemma 3.4), we get

$$
\begin{aligned}
h(x, x) & \leq h_{T_{l}}\left(x, \gamma\left(T_{l}\right)\right)+h\left(\gamma\left(T_{l}\right), \gamma\left(-T_{l}\right)\right)+h_{T_{l}}\left(\gamma\left(-T_{l}\right), x\right) \\
& =h_{T_{l}}\left(\gamma\left(-T_{l}\right), x\right)+h_{T_{l}}\left(x, \gamma\left(T_{l}\right)\right)+h\left(\beta_{l}, \alpha_{l}\right)+h\left(\gamma\left(T_{l}\right), \gamma\left(-T_{l}\right)\right)-h\left(\beta_{l}, \alpha_{l}\right) \\
& \leq h_{T_{l}}\left(\gamma\left(-T_{l}\right), x\right)+h_{T_{l}}\left(x, \gamma\left(T_{l}\right)\right)+h\left(\beta_{l}, \alpha_{l}\right)+K\left[d\left(\gamma\left(T_{l}\right), \beta_{l}\right)+d\left(\gamma\left(-T_{l}\right), \alpha_{l}\right)\right] \\
& \leq h_{T_{l}}\left(\gamma\left(-T_{l}\right), x\right)+h_{T_{l}}\left(x, \gamma\left(T_{l}\right)\right)+h\left(\beta_{l}, \alpha_{l}\right)+K \eta \\
& =h_{T_{l}}\left(\gamma\left(-T_{l}\right), x\right)+h_{T_{l}}\left(x, \gamma\left(T_{l}\right)\right)-h\left(\alpha_{l}, \beta_{l}\right)+K \eta \\
& \leq h_{T_{l}}\left(\gamma\left(-T_{l}\right), x\right)+h_{T_{l}}\left(x, \gamma\left(T_{l}\right)\right)-h\left(\gamma\left(-T_{l}\right), \gamma\left(T_{l}\right)\right)+2 K \eta \\
& =\left(u(x)-u\left(\gamma\left(-T_{l}\right)\right)\right)+\left(u\left(\gamma\left(T_{l}\right)\right)-u(x)\right)-h\left(\gamma\left(-T_{l}\right), \gamma\left(T_{l}\right)\right)+2 K \eta \\
& \leq u\left(\gamma\left(T_{l}\right)\right)-u\left(\gamma\left(-T_{l}\right)\right)-h\left(\gamma\left(-T_{l}\right), \gamma\left(T_{l}\right)\right)+2 K \eta \leq 2 K \eta,
\end{aligned}
$$

where for the last inequality we used (3.3). By the arbitrariness of $\eta$ this shows that $h(x, x)=0$, which implies that $x$ belongs to $\mathcal{A}(H)$, a contradiction. This proves the upper-semicontinuity of the Aubry set, and conclusion follows easily from Proposition 2.18.

Thanks to Lemma 3.2, it is now sufficient to show a density result, that is, given a Tonelli Hamiltonian $H$ of class $C^{2}$ and $\epsilon>0$, there is $V \in C^{2}(M)$ with $\|V\|_{C^{2}(M)}<\epsilon$ such that the Aubry set of $H+V$ is minimal and hyperbolic.

\subsection{The density part}

Let us fix a $C^{2}$ Tonelli Hamiltonian $H$. First of all, up to adding a small potential (in the $C^{2}$ topology) we may assume that the Aubry set $\tilde{\mathcal{A}}(H)$ is minimal, that is all its orbits are dense in $\widetilde{\mathcal{A}}(H)$ (see $[19, \S 5.1]$ where we explain how to add a potential to reduce the size of the Aubry set). We can also assume that $\tilde{\mathcal{A}}(H)$ is not an equilibrium point or a periodic orbit, as otherwise we may add an arbitrarily small potential to make it hyperbolic (see [12, Theorem $\mathrm{D}]^{2}$ ). Thus, the critical energy level

$$
\Sigma:=\left\{\theta=(x, p) \in T^{*} M \mid H(x, p)=\mathbf{c}[H]\right\} \subset T^{*} M
$$

satisfies the assumptions of Section 2.4. Since we work on a surface, two cases may appear. Either the positive and negative Green bundles along $\tilde{\mathcal{A}}(H)$ satisfy

$$
G_{\theta}^{-} \cap G_{\theta}^{+}=\mathbb{R} X_{H}(\theta) \quad \forall \theta \in \tilde{\mathcal{A}}(H),
$$

or

$$
G_{\bar{\theta}}^{+}=G_{\bar{\theta}}^{-} \quad \text { for some } \quad \bar{\theta} \in \tilde{\mathcal{A}}(H) .
$$

In the first case (when (3.6) holds), the hyperbolicity of $\tilde{\mathcal{A}}(H)$ follows from Proposition 2.19. Indeed, consider the projection $\Psi_{t}$ of the differential of the Hamiltonian flow to the bundle

$$
\mathbf{N}_{\theta}:=\left\{\xi \in T_{\theta} \Sigma \mid\left\langle D_{\theta} \pi^{*}(\xi), D_{\theta} \pi^{*}\left(X_{H}(\theta)\right)\right\rangle_{\pi^{*}(\theta)}=0\right\}
$$

that is $\Psi_{t}:=\left.\Lambda \circ D \phi_{t}^{H}\right|_{\mathbf{N}}$ where $\Lambda: T \Sigma \rightarrow \mathbf{N}$ is the projection along the direction of the $X_{H}$ :

$$
\Lambda \xi=\xi+\beta(\xi) X_{H}(\theta) \quad \text { with } \beta(\xi) \in \mathbb{R} \text { such that } \Lambda \xi \in \mathbf{N}_{\theta} .
$$

Since the Green bundles are always transverse, the restriction of $\Psi_{t}$ to $\tilde{\mathcal{A}}(H)$ is quasi-hyperbolic (cf. [12, Corollary 2.3(d)]). Therefore, since we are assuming that $\tilde{\mathcal{A}}(H)$ is minimal, Proposition 2.19 implies that $\Psi_{t}$ is a hyperbolic action and then $\tilde{\mathcal{A}}(H)$ is a hyperbolic set.

\footnotetext{
${ }^{2}$ Notice that Contreras and Iturriaga require the Hamiltonian to be at least of class $C^{3}$, but the proof of their Theorem $\mathrm{D}$ works under $C^{2}$ regularity.
} 
In the second case (when (3.7) holds), the results in Section 2.4 show that critical solutions restricted to the Aubry sets are $C^{2}$ at $x=\pi^{*}(\theta)$. As we will show below, this property allows us to implement the techniques developed in $[19,20]$ to close the orbit of $\bar{\theta}$ into a periodic orbit. However, the construction of a critical subsolution for the new Hamiltonian (which is unavoidable to close the orbit into an genuine Aubry set) becomes much more difficult than in $[19,20]$ because of the lack of regularity of critical solutions in a neighborhood of the orbit passing through $x$ (in $[19,20]$, the authors had to assume extra regularity on a critical solution to make their argument work). Still, thanks to the preparatory results on semiconcave and BV functions given in Section 2.7, we will be able to perform such a construction and make the whole proof work. So, the goal of the next section is to prove the following result, from which Theorem 1.1 follows.

Proposition 3.3. Let $H: T^{*} M \rightarrow \mathbb{R}$ be a Hamiltonian of class $C^{2}$, and assume that $\operatorname{dim} M=$ 2. Let $\mathcal{V}$ be a neighborhood of 0 in $C^{2}(M)$ and $\bar{\theta} \in \tilde{\mathcal{A}}(H)$ with $X_{H}(\bar{\theta}) \neq 0$ be such that $G_{\bar{\theta}}^{+}=G_{\bar{\theta}}^{-}$. Then there exists $V \in \mathcal{V}$ such that the Aubry set associated to the Hamiltonian $H+V$ is a hyperbolic periodic orbit (in its energy level).

\subsection{Proof of Proposition 3.3}

From now on, we assume that the Aubry set $\tilde{\mathcal{A}}(H)$ is a minimal set which is neither an equilibrium point nor a periodic orbit. Without loss of generality, up to adding a constant to $H$ (which does not change the dynamics), we can assume that $\mathbf{c}[H]=0$. Let $L$ denote the Lagrangian associated to $H$. Given $\epsilon>0$, our goal is to find a potential $V: M \rightarrow \mathbb{R}$ of class $C^{2}$ with $\|V\|_{C^{2}}<\epsilon$, together with a Lipschitz function $v_{V}: M \rightarrow \mathbb{R}$, and a curve $\gamma:\left[0, T^{\prime}\right] \rightarrow M$ with $\gamma(0)=\gamma\left(T^{\prime}\right)$, such that the following properties are satisfied:

(P1) $H_{V}\left(x, d v_{V}(x)\right) \leq 0$ for a.e. $x \in M$.

(P2) $\int_{0}^{T^{\prime}} L_{V}(\gamma(t), \dot{\gamma}(t)) d t=0$.

Indeed, as explained in $[19, \S 5.1]$ (see also [21]), such a construction yields a Hamiltonian $H_{V}$ whose Aubry set contains the closed orbit $\gamma\left(\left[0, T^{\prime}\right]\right)$. Adding a small non-positive potential vanishing exactly on $\gamma\left(\left[0, T^{\prime}\right]\right.$ ), we get a Hamiltonian whose Aubry set is a periodic orbit (see $[19, \S 5.1]$ for more details). Moreover, as shown by Contreras and Iturriaga [12, Theorem D], we can add a potential, small in the $C^{2}$ topology, which preserves the periodic orbit and makes it a hyperbolic Aubry set. Hence, we are left with finding $V, v_{V}$, and $\gamma$ such that (P1) and (P2) hold.

Fix $\epsilon>0$, and let $\bar{\theta}=(\bar{x}, \bar{p}) \in \tilde{\mathcal{A}}(H)$ be as in the statement of Proposition 3.3. Let us denote by $\bar{\theta}(\cdot)=(\bar{\gamma}(\cdot), \bar{p}(\cdot))$ the orbit of $\bar{\theta}$ by the Hamiltonian flow, and by $\bar{\Pi} \subset M$ a local section (that is, a smooth curve) which is transverse to $\bar{\gamma}$ at $t=0$. Let $u: M \rightarrow \mathbb{R}$ be a critical solution for $H$. Recall that $u$ is differentiable on the projected Aubry set $\mathcal{A}(H)$, and that the restriction of $d u$ to $\mathcal{A}(H)$ is Lipschitz (see Proposition 2.5).

The following lemma will be needed to apply Proposition 2.7.

Lemma 3.4. Let $c_{0}>0$ be as in Proposition 2.7. There exists $\bar{t}>0$ such that, on any time interval of the form $\left[t_{0}, t_{0}+\bar{t}\right]$ there is a time $t^{\prime} \in\left[t_{0}, t_{0}+\bar{t}\right]$ such that

$$
\frac{d}{d t}\left\{u\left(\phi_{t}^{H}(\bar{x}, \bar{p})\right)\right\}_{\mid t=t^{\prime}}=\left\langle d u\left(\bar{\gamma}\left(t^{\prime}\right)\right), \dot{\bar{\gamma}}\left(t^{\prime}\right)\right\rangle \geq-c_{0} .
$$

Proof of Lemma 3.4. If not

$$
u\left(\bar{\gamma}\left(t_{0}+\bar{t}\right)\right)-u\left(\bar{\gamma}\left(t_{0}\right)\right)=\int_{t_{0}}^{t_{0}+\bar{t}}\langle d u(\bar{\gamma}(s)), \dot{\bar{\gamma}}(s)\rangle d s \leq-\int_{t_{0}}^{t_{0}+\bar{t}} c_{0}=-c_{0} \bar{t} .
$$

Since $u$ is bounded, this is impossible if $\bar{t}$ is sufficiently large. 
Up to replace $H$ by $4 H / \bar{t}$, we can assume that the constant $\bar{t}$ appearing in the previous lemma satisfies ${ }^{3}$

$$
\bar{t}=1 / 4 .
$$

Let us take $T>0$ to be fixed. Since $\bar{\gamma}$ can never intersect itself, there exist an open neighborhood $\mathcal{U}$ of $\bar{\gamma}([0, T])$ in $M$, and a $C^{2}$ diffeomorphism $\Phi: \mathcal{U} \rightarrow \mathcal{U}^{\prime}:=\Phi(\mathcal{U}) \subset \mathbb{R}^{2}$, such that, in the new system of coordinates, the curve $\Phi\left(\bar{\gamma}_{\mid[0, T]}\right)$ is a straight segment. Hence, using still $\bar{\gamma}$ instead of $\Phi(\bar{\gamma})$ to denote this curve (by a slight abuse of notation), we can assume that

$\left(\pi_{1}\right) \bar{\gamma}(t)=\left(t e_{1}, 0\right)$ for any $t \in[-1, T] ;$

$\left(\pi_{2}\right)[-1, T] \times[-\rho, \rho] \subset \mathcal{U}^{\prime}$.

(Here and in the sequel, $\left(e_{1}, e_{2}\right)$ denotes the canonical basis in $\mathbb{R}^{2}$.) Also, in this new set of coordinates, we can see $H$ as a Hamiltonian on $T^{*} \mathcal{U}^{\prime} \subset T^{*} \mathbb{R}^{2}=\mathbb{R}^{2} \times\left(\mathbb{R}^{2}\right)^{*}$, and the critical solution $u$ as a semiconcave function on $\mathbb{R}^{2}$. We set

$$
\Pi_{r}^{t}:=\left\{\left(t e_{1}, y\right) \mid y \in[-r, r]\right\} \quad \forall t, r \in \mathbb{R} .
$$

The intersection of the Aubry set (resp. projected Aubry set) with $T^{*} \mathcal{U}$ (resp. with $\mathcal{U}$ ) is transported by $\Phi$. Let us denote by $\tilde{\mathcal{A}}$ and $\mathcal{A}$ their respective images in $T^{*} \mathcal{U}^{\prime}$ and $\mathcal{U}^{\prime}$. The Green bundles $G_{\phi_{t}^{H}(\bar{\theta})}^{+}=G_{\phi_{t}^{H}(\bar{\theta})}^{-}$for $t \in[-1, T]$, and $G_{\theta}^{+}$with $\theta \in T^{*} \mathcal{U}$, are also transported by $\Phi$. We denote them respectively by $G_{t}$ and $G_{\theta}^{+}$in $T\left(T^{*} \mathcal{U}^{\prime}\right)$. We now apply (3.7) and Corollary 2.15 to deduce that, up to reduce the size of $\rho$ and $\mathcal{U}^{\prime}$, there is a function $f: \mathcal{U}^{\prime} \rightarrow \mathbb{R}$ of class $C^{1,1}$ such that the 1 -form $\Psi:=d f$ on $\mathcal{U}^{\prime}$ satisfies the following properties:

$\left(\pi_{3}\right) \Psi$ is $C^{1}$ along $\bar{\gamma}([-1, T])$;

$\left(\pi_{4}\right) \tilde{\mathcal{A}} \cap T^{*} \mathcal{U}^{\prime} \subset \operatorname{Graph}(\Psi)$;

$\left(\pi_{5}\right)$ for every $t \in[-1, T], G_{t}=\operatorname{Graph}\left(L_{t}:=d_{\bar{\gamma}(t)} \Psi\right) \subset \mathbb{R}^{2} \times\left(\mathbb{R}^{2}\right)^{*}$.

\subsubsection{Some preliminary regularity estimates on $u$}

Let us recall that, thanks to Proposition 2.3, $u$ is semiconcave, so the discussion in Section 2.7 (see in particular Section 2.7.2) applies. Also, since $\bar{\gamma}([0, T])=\left\{t e_{1}\right\}_{t \in[0, T]}\left(\right.$ see $\left(\pi_{1}\right)$ ) and $t e_{1} \in \mathcal{A}$ (hence $u$ is differentiable at $y_{1}$, see Proposition 2.5), by upper-semicontinuity of the limiting differential of semiconcave functions there is a modulus of continuity $\omega: \mathbb{R}^{+} \rightarrow \mathbb{R}^{+}$ (that is, $\omega$ is nondecreasing with $\lim _{r \downarrow 0} \omega(r)=0$ ), possibly depending on $T$, such that

$$
\left|(x, p)-\left(t e_{1}, \nabla u\left(t e_{1}\right)\right)\right| \leq \omega(r) \quad \forall x \in \Pi_{r}^{t}, p \in D_{x}^{*} u(x), t \in[0, T], r \in(0, \rho)
$$

and $\left(\right.$ since $\frac{\partial H}{\partial p}\left(t e_{1}, \nabla u\left(t e_{1}\right)\right)=e_{1}$, see $\left.\left(\pi_{1}\right)\right)$

$$
\left|\frac{\partial H}{\partial p}(x, p)-e_{1}\right| \leq \omega(r) \quad \forall x \in \Pi_{r}^{t}, p \in D_{x}^{*} u(x), t \in[0, T], r \in(0, \rho) .
$$

As in $(2.20)$, for a.e. $t \in[0, T]$ we define the function $w_{t}:[-\rho, \rho] \rightarrow \mathbb{R}^{n}$ by

$$
w_{t}(s):=\nabla u(t, s) \quad \text { for a.e. } s \in[-\rho, \rho],
$$

\footnotetext{
${ }^{3}$ Notice that the flow of the Hamiltonian $\bar{H}(x, p):=4 H(x, p) / \bar{t}$ is just a reparameterization of the flow of $H$, and $u$ is still a solution of $\bar{H}(x, d u)=0$. The advantage of choosing $\bar{t}=1 / 4$ is that later we will be able to connect trajectories over time intervals of length 1 instead of $\bar{t}$.
} 
and we recall the following decomposition for $D w_{t}$ (see Section 2.7):

$$
D w_{t}=\nabla w_{t} d s+D_{\mathcal{S}} w_{t},
$$

where $\nabla w_{t} d s$ is absolutely continuous and $D_{\mathcal{S}} w_{t}$ is singular with respect to $d s$. It follows from (2.29)-(2.31) that

$$
\left|D_{\mathcal{S}} w_{t}\right| \leq 2\left|D_{\mathcal{S}} w_{t} \cdot e_{2}\right| \quad \text { inside } \mathcal{U}^{\prime} \text {, for a.e. } t \in[0, T] .
$$

Also, Proposition 2.17 combined with the upper semicontinuity of the positive Green bundle provides an upper bound on $D^{2} u$ in a neighborhood of a the curve $\bar{\gamma}([0, T])$. More precisely, we recall that $\left\langle D_{\mathcal{S}}^{2} v \cdot e, e\right\rangle$ is a nonpositive measure for any vector $e \in \mathbb{R}^{n}$ (see Section 2.7). Also, by $\left(\pi_{5}\right)$ and Propositions 2.10 and 2.17 we deduce that there exists a modulus of continuity $\omega^{\prime}: \mathbb{R}^{+} \rightarrow \mathbb{R}^{+}$, possibly depending on $T$, such that, for a.e. $t \in[0, T]$,

$$
\nabla^{2} u(x) \leq L_{t}+\omega^{\prime}(r) \mathrm{Id} \quad \text { for a.e. } x \in \Pi_{r}^{t} .
$$

(Recall that $\nabla^{2} u$ denotes the pointwise Hessian of $u$, which exists almost everywhere.) We denote by $\mathscr{O}$ the orbit of $\bar{\gamma}$ in $\mathcal{U}^{\prime}$, that is $\mathscr{O}:=\bar{\gamma}(\mathbb{R}) \cap \mathcal{U}^{\prime}$.

In the next lemma we use (3.7) to show that, for a.e. $t, D w_{t}$ is close in total variation to a constant matrix. From now on, we always denote a modulus of continuity by $\omega$ and a positive constant by $C$, their values might change from line to line but otherwise they depend only on $T$ and the data (i.e., $H, u$, etc.).

Lemma 3.5. Let $\Psi$ be as in $\left(\pi_{3}\right)-\left(\pi_{5}\right)$. There exist a modulus of continuity $\omega: \mathbb{R}^{+} \rightarrow \mathbb{R}^{+}$and a constant $C>0$ such that the following properties hold for any $r \in(0, \rho]$ :

(i) For a.e. $t \in[0, T]$, for every $y_{1}=\left(t, \ell_{1}\right), y_{2}=\left(t, \ell_{2}\right) \in \mathscr{O} \cap \Pi_{r}^{t}$ with $\ell_{2}>\ell_{1}$,

$$
\int_{\ell_{1}}^{\ell_{2}}\left|\nabla w_{t}(s)-L_{t} \cdot e_{2}\right| d s+\int_{\ell_{1}}^{\ell_{2}} d\left|D_{\mathcal{S}} w_{t}\right|(s) \leq \omega(r)\left|\ell_{2}-\ell_{1}\right| \text {. }
$$

(ii) For every $y_{1}, y_{2} \in \mathscr{O} \cap \Pi_{\rho}^{T}$ there exists a family of matrices $\left\{M_{-t}\right\}_{t \in[0, T]}$, with

$$
\left|M_{-t}\right|+\left|\left(M_{-t}\right)^{-1}\right| \leq C
$$

such that the following holds for any constant $N \geq 1$ : for every $z, z^{\prime} \in \Pi_{\rho}^{T} \cap\left[y_{1}, y_{2}\right]$ such that $u$ is differentiable at $z, z^{\prime}$ and $\left|z^{\prime}-z\right| \geq \frac{\left|y_{1}-y_{2}\right|}{N}$, we have

$$
\left|\pi^{*}\left(\phi_{-t}^{H}(z, \nabla u(z))\right)-\pi^{*}\left(\phi_{-t}^{H}\left(z^{\prime}, \nabla u\left(z^{\prime}\right)\right)\right)-M_{-t}\left(z-z^{\prime}\right)\right| \leq N \omega(r)\left|z-z^{\prime}\right| .
$$

Proof of Lemma 3.5. We begin by observing that $\left|y_{2}-y_{1}\right|=\left|\ell_{2}-\ell_{1}\right|$. Since the graph of $\nabla u$ restricted to $\bar{\gamma}([0, T])=\left\{t e_{1}\right\}_{t \in[0, T]}$ is contained inside the graph of $\Psi$ and the latter is $C^{1}$ there (see $\left(\pi_{1}\right)$ and $\left.\left(\pi_{3}\right)\right)$, for a.e. $t \in[0, T]$ we get

$$
\begin{aligned}
\left|w_{t}\left(\ell_{2}\right)-w_{t}\left(\ell_{1}\right)-L_{t} \cdot\left(y_{2}-y_{1}\right)\right| & =\left|\Psi\left(y_{2}\right)-\Psi\left(y_{1}\right)-d_{\bar{\gamma}(t)} \Psi \cdot\left(y_{2}-y_{1}\right)\right| \\
& =\left|\int_{0}^{1} d_{y_{1}+s\left(y_{2}-y_{1}\right)} \Psi \cdot\left(y_{2}-y_{1}\right) d s-d_{\bar{\gamma}(t)} \Psi \cdot\left(y_{2}-y_{1}\right)\right| \\
& \leq \int_{0}^{1}\left|d_{y_{1}+s\left(y_{2}-y_{1}\right)} \Psi-d_{\bar{\gamma}(t)} \Psi\right|\left|y_{2}-y_{1}\right| d s \\
& \leq \omega(r)\left|\ell_{2}-\ell_{1}\right|,
\end{aligned}
$$


for some modulus of continuity $\omega: \mathbb{R}^{+} \rightarrow \mathbb{R}^{+}$. So, rewriting the above expression using the fundamental theorem of calculus (see $(2.21)$ ), for a.e. $t \in[0, T]$ we have (observe that $\left.\frac{y_{2}-y_{1}}{\left|y_{2}-y_{1}\right|}=e_{2}\right)$

$$
\left|\int_{\ell_{1}}^{\ell_{2}}\left[\nabla w_{t}(s)-L_{t} \cdot e_{2}\right] d s+\int_{\ell_{1}}^{\ell_{2}} d\left[D_{\mathcal{S}} w_{t}\right](s)\right| \leq \omega(r)\left|\ell_{2}-\ell_{1}\right|,
$$

which implies in particular that

$$
\left|\int_{\ell_{1}}^{\ell_{2}}\left[\nabla w_{t}(s) \cdot e_{2}-\left\langle L_{t} \cdot e_{2}, e_{2}\right\rangle\right] d s+\int_{\ell_{1}}^{\ell_{2}} d\left[D_{\mathcal{S}} w_{t} \cdot e_{2}\right](s)\right| \leq \omega(r)\left|\ell_{2}-\ell_{1}\right| .
$$

This estimate combined with $(2.23),(3.13)$, and (2.25), gives

$$
\int_{\ell_{1}}^{\ell_{2}}\left|\nabla w_{t}(s) \cdot e_{2}-\left\langle L_{t} \cdot e_{2}, e_{2}\right\rangle\right| d s+\int_{\ell_{1}}^{\ell_{2}} d\left|D_{\mathcal{S}} w_{t} \cdot e_{2}\right|(s) \leq \omega(r)\left|\ell_{2}-\ell_{1}\right| \quad \text { for a.e. } t \in[0, T],
$$

which shows that $D w_{t} \cdot e_{2}$ is $L^{1}$-close to $\left\langle L_{t} \cdot e_{2}, e_{2}\right\rangle$.

We now need to control $D w_{t} \cdot e_{1}$. For this, we first apply (3.12) to obtain that the singular part of $D w_{t}$ is controlled by $D_{\mathcal{S}} w_{t} \cdot e_{2}$ : indeed (3.12) and the bound above imply

$$
\int_{\ell_{1}}^{\ell_{2}} d\left|D_{\mathcal{S}} w_{t}\right|(s) \leq 2 \int_{\ell_{1}}^{\ell_{2}} d\left|D_{\mathcal{S}} w_{t} \cdot e_{2}\right|(s) \leq 2 \omega(r)\left|\ell_{2}-\ell_{1}\right| \quad \text { for a.e. } t \in[0, T] .
$$

Hence it suffices to control only the absolutely continuous part of $D w_{t}$.

Recall that, thanks to (2.23), for a.e. $t \in[0, T]$ we have

$$
\nabla w_{t}(s)=\nabla^{2} u(t, s) \cdot e_{2} \quad \text { for a.e. } s \in[-r, r],
$$

where $\nabla^{2} u$ is the Hessian of $u$, which exists at almost every point. Hence it suffices to prove the closeness of $\nabla w_{t}$ to $L_{t} \cdot e_{2}$ only at points where $u$ is twice differentiable.

For every $x_{\ell}:=(t, \ell) \in \Pi_{r}^{t}$ where $u$ is twice differentiable, consider the curve

$$
\left(x_{\ell}(\tau), p_{\ell}(\tau)\right):=\phi_{\tau}^{H}\left(x_{\ell}, \nabla u\left(x_{\ell}\right)\right) .
$$

It follows from $(3.10)$ and $\left(\pi_{1}\right)$ that

$$
\left|x_{\ell}(-\tau)-(t-\tau) e_{1}\right| \leq \omega(r) \quad \forall t \in[0, T], \tau \in[0,1] .
$$

Also, since the trajectories do not cross backward in time, $u$ is differentiable along them, and $p_{\ell}(-\tau)=\nabla u\left(x_{\ell}(-\tau)\right)$ (see Proposition 2.4), we have (here we use $\dot{x}_{s}(\tau)$ to denote the derivative with respect to $\tau)$

$$
\frac{d}{d \tau}\left[p_{\ell}(-\tau)\right]=\frac{d}{d \tau}\left[\nabla u\left(x_{\ell}(-\tau)\right)\right]=-\nabla^{2} u\left(x_{\ell}(-\tau)\right) \cdot \dot{x}_{\ell}(-\tau) \quad \forall \tau \in[0,1],
$$

Since $p_{\ell}$ is uniformly bounded and solves the Hamiltonian system, also $\frac{d}{d \tau}\left[p_{\ell}(-\tau)\right]$ is uniformly bounded, hence we have

$$
\left|\left\langle\nabla^{2} u\left(x_{\ell}(-\tau)\right) \cdot e, \dot{x}_{\ell}(\tau)\right\rangle-\left\langle\nabla^{2} u\left(x_{\ell}(0)\right) \cdot e, \dot{x}_{\ell}(0)\right\rangle\right| \leq C \tau|e| \quad \forall e \in \mathbb{R}^{n}, \forall \tau \in[0,1] .
$$


To simplify the notation, set $x_{s}:=x_{\ell_{1}+s\left(\ell_{2}-\ell_{1}\right)}$. Then, it follows from (3.15)-(3.16) and the smoothness in $\tau$ of the curves $\tau \mapsto x_{s}(\tau)$ that, for every $\tau \in[0,1]$,

$$
\begin{aligned}
& \int_{0}^{1}\left|\nabla w_{t}\left(\ell_{1}+s\left(\ell_{2}-\ell_{1}\right)\right) \cdot \dot{x}_{s}(0)-\left\langle L_{t} \cdot e_{2}, \dot{x}_{s}(0)\right\rangle\right| d s \\
= & \int_{0}^{1}\left|\left\langle\nabla^{2} u\left(x_{s}(0)\right) \cdot e_{2}, \dot{x}_{s}(0)\right\rangle-\left\langle L_{t} \cdot e_{2}, \dot{x}_{s}(0)\right\rangle\right| d s \\
\leq & \int_{0}^{1}\left|\frac{1}{\tau} \int_{-\tau}^{0}\left\langle\nabla^{2} u\left(x_{s}(\sigma)\right) \cdot \dot{x}_{s}(\sigma), e_{2}\right\rangle d \sigma-\left\langle L_{t} \cdot e_{2}, \dot{x}_{s}(0)\right\rangle\right| d s \\
& +\int_{0}^{1} \frac{1}{\tau} \int_{-\tau}^{0}\left|\left\langle\nabla^{2} u\left(x_{s}(\sigma)\right) \cdot e_{2}, \dot{x}_{s}(\sigma)\right\rangle-\left\langle\nabla^{2} u\left(x_{s}(0)\right) \cdot e_{2}, \dot{x}_{s}(0)\right\rangle\right| d \sigma d s \\
\leq & \int_{0}^{1}\left|\left\langle\frac{\nabla u\left(x_{s}(0)\right)-\nabla u\left(x_{s}(-\tau)\right)}{\tau}, e_{2}\right\rangle-\left\langle L_{t} \cdot e_{2}, \dot{x}_{s}(0)\right\rangle\right| d s+C \tau .
\end{aligned}
$$

By $\left(\pi_{1}\right)$ and $\left(\pi_{5}\right)$ (note that $\nabla u$ varies smoothly along $\bar{\gamma}([0, T]$ ), since it solves the Hamiltonian system) we have

$$
\left|\frac{\nabla u\left(t e_{1}\right) \cdot e_{2}-\nabla u\left((t-\tau) e_{1}\right) \cdot e_{2}}{\tau}-\left\langle L_{t} \cdot e_{1}, e_{2}\right\rangle\right| \leq C \tau \quad \forall \tau \in[0,1] .
$$

Hence, by (3.10), (3.11), and (3.17), for every $\tau \in[0,1]$ we get

$$
\int_{0}^{1}\left|\nabla w_{t}\left(\ell_{1}+s\left(\ell_{2}-\ell_{1}\right)\right) \cdot \dot{x}_{s}(0)-\left\langle L_{t} \cdot e_{2}, \dot{x}_{s}(0)\right\rangle\right| d s \leq C \tau+\frac{\omega(r)}{\tau} .
$$

Thus, choosing $\tau:=\sqrt{\omega(r)}$ and using that $\left|\dot{x}_{s}(0)-e_{1}\right| \leq \omega(r)$ and that $L_{t}$ is bounded (since $u$ is universally $C^{1,1}$ on the Aubry set), we get

$$
\begin{aligned}
\frac{1}{\left|\ell_{2}-\ell_{1}\right|} \int_{\ell_{1}}^{\ell_{2}}\left|\nabla w_{t}(s) \cdot e_{1}-\left\langle L_{t} \cdot e_{2}, e_{1}\right\rangle\right| d s & =\int_{0}^{1}\left|\nabla w_{t}\left(\ell_{1}+s\left(\ell_{2}-\ell_{1}\right)\right) \cdot e_{1}-\left\langle L_{t} \cdot e_{2}, e_{1}\right\rangle\right| d s \\
& \leq C \sqrt{\omega(r)},
\end{aligned}
$$

concluding the proof of (i).

Let us now prove the second assertion. To simplify the notation, for a.e. $\bar{t} \in[T-1, T]$ we define the functions ${ }^{4}$

$\psi_{-t}^{\bar{t}}(s):=\pi^{*}\left(\phi_{-t}^{H}(z, \nabla u(z))\right)=\pi^{*}\left(\phi_{-t}^{H}\left((\bar{t}, s), w_{\bar{t}}(s)\right)\right), \quad$ for a.e. $z=(\bar{t}, s) \in \Pi_{r}^{\bar{t}}, \forall t \in[0, T]$.

By the chain-rule formula for BV functions [2, Theorem 3.96], the following hold: if we decompose the distributional derivative $D \psi_{-t}^{\bar{t}}$ into its absolutely continuous part $\nabla \psi_{-t}^{\bar{t}}$ and its singular part $D_{\mathcal{S}} \psi_{-t}^{\bar{t}}$, we have

$$
\nabla \psi_{-t}^{\bar{t}}(s)=d \pi^{*}\left(\phi_{-t}^{H}\left((\bar{t}, s), w_{\bar{t}}(s)\right)\right) \cdot\left(\partial_{x_{2}} \phi_{-t}^{H}\left((\bar{t}, s), w_{\bar{t}}(s)\right)+\partial_{p} \phi_{-t}^{H}\left((\bar{t}, s), w_{\bar{t}}(s)\right) \cdot \nabla w_{\bar{t}}(s)\right)
$$

for a.e. $s \in[-r, r]$, and

$$
\left|D_{\mathcal{S}} \psi_{-t}^{\bar{t}}\right| \leq C\left|D_{\mathcal{S}} w_{\bar{t}}\right| \cdot
$$

Given $z, z^{\prime} \in \Pi_{r}^{\bar{t}}$, let us denote by $\int_{z}^{z^{\prime}} d \mu$ the integral of a measure $\mu$ over the segment joining $z$ to $z^{\prime}$. Then, by (i) and (3.10), for every $\tau \in[0, T]$ we have

$$
\begin{aligned}
\int_{y_{1}}^{y_{2}} d\left|D \psi_{-t}^{\bar{t}}-M_{-t}^{\bar{t}} \cdot e_{2}\right| & =\int_{y_{1}}^{y_{2}}\left|\nabla \psi_{-t}^{\bar{t}}(s)-M_{-t}^{\bar{t}} \cdot e_{2}\right| d s+\int_{y_{1}}^{y_{2}} d\left|D_{\mathcal{S}} \psi_{-t}^{\bar{t}}\right|(s) \\
& \leq \omega(r)\left|y_{2}-y_{1}\right|,
\end{aligned}
$$

\footnotetext{
${ }^{4}$ Notice that, since $u$ is differentiable a.e., for a.e. $t \in[0, T]$ we have that $u$ is differentiable at a.e. $z \in\left[y_{1}, y_{2}\right]$.
} 
where

$$
M_{-t}^{\bar{t}}:=d \pi^{*}\left(\phi_{-t}^{H}\left(\bar{t} e_{1}, e_{1}\right)\right) \cdot\left(\partial_{x} \phi_{-t}^{H}\left(\bar{t} e_{1}, e_{1}\right)+\partial_{p} \phi_{-t}^{H}\left(\bar{t} e_{1}, e_{1}\right) L_{\bar{t}}\right),
$$

and we used that $\left|L_{\bar{t}}\right|$ is universally bounded (because $u$ is universally $C^{1,1}$ on the Aubry set) to estimate

$$
\begin{aligned}
& \mid \partial_{p} \phi_{-t}^{H}\left((\bar{t}, s), w_{\bar{t}}(s)\right) \cdot \nabla w_{\bar{t}}(s)-\partial_{p} \phi_{-t}^{H}\left(\bar{t} e_{1}, e_{1}\right) L_{\bar{t}} \cdot e_{2} \mid \\
& \leq\left|\partial_{p} \phi_{-t}^{H}\left((\bar{t}, s), w_{\bar{t}}(s)\right) \cdot\left(\nabla w_{\bar{t}}(s)-L_{\bar{t}} \cdot e_{2}\right)\right| \\
& \quad+\left|\left(\partial_{p} \phi_{-t}^{H}\left((\bar{t}, s), w_{\bar{t}}(s)\right)-\partial_{p} \phi_{-t}^{H}\left(\bar{t} e_{1}, e_{1}\right)\right) L_{\bar{t}} \cdot e_{2}\right|
\end{aligned}
$$

The boundedness of $\left|L_{\bar{t}}\right|$ implies also that the norm $M_{-t}^{\bar{t}}$ is bounded on $[0, T]$ by a constant depending only on $T$. Also, since $u$ is semiconcave, a simple Gronwall argument shows that the backward flow $t \mapsto \psi_{-t}(z)$ is not "too much contractive": there exists a universal constant $C>0$ such that

$$
\left|\psi_{-t}^{\bar{t}}(z)-\psi_{-t}^{\bar{t}}\left(z^{\prime}\right)\right| \geq e^{-C t}\left|z-z^{\prime}\right| \quad \forall t \in[0, T],
$$

from which we deduce that $\left|\left(M_{-t}^{\bar{t}}\right)^{-1}\right| \leq e^{C T}$ and that the trajectories cannot cross backward in time. Also, from (3.18) and the assumption $\left|z^{\prime}-z\right| \geq \frac{\left|y_{2}-y_{1}\right|}{N}$ we deduce that

$$
\begin{aligned}
\left|\psi_{-t}^{\bar{t}}(z)-\psi_{-t}^{\bar{t}}\left(z^{\prime}\right)-M_{-t}^{\bar{t}}\left(z-z^{\prime}\right)\right| & \leq \int_{z^{\prime}}^{z} d\left|D \psi_{-t}^{\bar{t}}-M_{-t}^{\bar{t}} \cdot e_{2}\right| \leq \int_{y_{1}}^{y_{2}} d\left|D \psi_{-t}^{\bar{t}}-M_{-t}^{\bar{t}} \cdot e_{2}\right| \\
& \leq \omega(r)\left|y_{1}-y_{2}\right| \leq N \omega(r)\left|z^{\prime}-z\right|
\end{aligned}
$$

for a.e. $t \in[0, T]$ and a.e. $z, z^{\prime} \in \Pi_{r}^{t}$. By a simple approximation argument, the above estimate extends to $\bar{t}=T$ and every $z, z^{\prime} \in \Pi_{r}^{T}$ such that $u$ is differentiable at $z, z^{\prime}$, concluding the proof with $M_{-t}:=M_{-t}^{T}$.

The following bound will be crucial to estimate the action.

Lemma 3.6. There exist a modulus of continuity $\omega: \mathbb{R}^{+} \rightarrow \mathbb{R}^{+}$and a constant $K^{\prime}>0$ such that the following holds: Let $y_{1}, y_{2} \in \mathscr{O} \cap \Pi_{\rho}^{t}$ for some $t \in[0, T]$. Then, for every $r \in(0, \rho]$, and for every $z_{1}, z_{2} \in \Pi_{r}^{t} \cap\left[y_{1}, y_{2}\right]$ such that $u$ is differentiable at $z_{1}, z_{2}$ and $\frac{\left|y_{1}-y_{2}\right|}{10 N} \leq\left|z_{1}-z_{2}\right| \leq \frac{\left|y_{1}-y_{2}\right|}{N}$,

$$
\left|u\left(z_{2}\right)-u\left(z_{1}\right)-\left\langle\nabla u\left(z_{1}\right), z_{2}-z_{1}\right\rangle\right| \leq K^{\prime} \frac{\left|y_{1}-y_{2}\right|^{2}}{N}
$$

$$
\left|\nabla u\left(z_{2}\right)-\nabla u\left(z_{1}\right)\right| \leq K^{\prime}\left(\omega(r)+\frac{1}{N}\right)\left|y_{1}-y_{2}\right| .
$$

Proof. Since $u$ is semiconcave, there exists a universal constant $C$ such that $v:=u-C|x|^{2}$ is concave. Since

$$
\left|\left(u\left(z_{2}\right)-u\left(z_{1}\right)-\left\langle\nabla u\left(z_{1}\right), z_{2}-z_{1}\right\rangle\right)-\left(v\left(z_{2}\right)-v\left(z_{1}\right)-\left\langle\nabla v\left(z_{1}\right), z_{2}-z_{1}\right\rangle\right)\right| \leq C\left|z_{1}-z_{2}\right|^{2} \leq C \frac{\left|y_{1}-y_{2}\right|^{2}}{N^{2}},
$$

it suffices to prove the result with $v$ in place of $u$.

By concavity of $v$, since $z_{2}-z_{1}$ is parallel to $y_{2}-y_{1}$, and $\left|z_{1}-z_{2}\right| \geq \frac{\left|y_{1}-y_{2}\right|}{10 N}$, we get

$$
\begin{aligned}
0 & \geq v\left(z_{2}\right)-v\left(z_{1}\right)-\left\langle\nabla v\left(z_{1}\right), z_{2}-z_{1}\right\rangle \\
& \geq\left\langle\nabla v\left(z_{2}\right)-\nabla v\left(z_{1}\right), z_{2}-z_{1}\right\rangle \\
& \geq\left\langle\nabla v\left(y_{2}\right)-\nabla v\left(y_{1}\right), z_{2}-z_{1}\right\rangle \\
& \geq \frac{1}{10 N}\left\langle\nabla v\left(y_{2}\right)-\nabla v\left(y_{1}\right), y_{2}-y_{1}\right\rangle \\
& \geq-C \frac{\left|y_{1}-y_{2}\right|^{2}}{N}
\end{aligned}
$$


where for the last estimate we used that $u$ (and hence $v$ ) is $C^{1,1}$ with a universal bound on the Aubry set. This proves (i).

For (ii), we recall that $\int_{z}^{z^{\prime}} d \mu$ denotes the integral of a measure $\mu$ over the segment joining $z$ to $z^{\prime}$. Hence, using the same notation as before, we apply Lemma 3.5(i) and use that $\left|L_{t}\right|$ is universally bounded (because of the $C^{1,1}$ regularity of $u$ on the Aubry set) to get, for a.e. $t \in[0, T]$,

$$
\begin{aligned}
\left|\nabla u\left(z_{2}\right)-\nabla u\left(z_{1}\right)\right| & \leq \int_{z_{1}}^{z_{2}} d\left|D w_{t}\right| \\
& \leq \int_{z_{1}}^{z_{2}} d\left|D w_{t}-L_{t} \cdot e_{2}\right|+\left|L_{t}\right|\left|z_{1}-z_{2}\right| \\
& \leq \int_{y_{1}}^{y_{2}} d\left|D w_{t}-L_{t} \cdot e_{2}\right|+C\left|z_{1}-z_{2}\right| \\
& \leq \omega(r)\left|y_{1}-y_{2}\right|+C \frac{\left|y_{1}-y_{2}\right|}{N} .
\end{aligned}
$$

By approximation, this estimate extends to every $t \in[0, T]$.

\subsubsection{The connection}

Given $y_{1}, y_{2} \in \mathbb{R}$, we set

$$
I^{1 / 3}\left(y_{1} ; y_{2}\right):=\left\{y \in \mathbb{R}\left|\operatorname{dist}\left(y,\left[y_{1}, y_{2}\right]\right)<\right| y_{1}-y_{2} \mid / 3\right\} .
$$

Lemma 5.2 in [20] (see also [3, Remarque 6.3.3]) applied with $n=1$ yields the following result:

Lemma 3.7. Let $\hat{r}>0$ and $Y$ be a finite set in $\mathbb{R}$ such that $B(0, \hat{r} / 12) \cap Y$ contains at least two points. Then there are $y_{1} \neq y_{2} \in Y$ such that the interval $I^{1 / 3}\left(y_{1} ; y_{2}\right)$ is included in $B(0, \hat{r})$ and does not intersect $Y \backslash\left\{y_{1}, y_{2}\right\}$.

Given $\hat{r} \in(0, \rho)$ small enough $(\hat{r}$ much smaller than $\rho$ and $\epsilon)$, let $T_{\hat{r}} \gg T$ be the first time such that $\bar{\gamma}\left(T_{\hat{r}}\right) \in \Pi_{r / 12}^{T}$, and define the set

$$
W:=\left\{w_{0}:=\bar{x}, w_{1}:=\bar{\gamma}\left(t_{1}\right), \ldots, w_{J}:=\bar{\gamma}\left(T_{\hat{r}}\right)\right\} \subset \mathcal{A}
$$

obtained by intersecting the curve

$$
\left[T, T_{\hat{r}}\right] \ni t \mapsto \bar{\gamma}(t)
$$

with $\Pi_{\hat{r}}^{T}$. We apply Lemma 3.7 with $Y=W \subset \Pi_{\hat{r}}^{T}$ to find two points

$$
\hat{y}_{1}=w_{j} \quad \text { and } \quad \hat{y}_{2}=w_{l} \quad \text { with } j>l
$$

which satisfy the properties described in the statement of the lemma. Set

$$
N:=\left\lfloor\frac{1}{\epsilon}\right\rfloor+1, \quad \eta:=2 N+1,
$$

and consider a sequence of points $\hat{z}_{1}, \ldots, \hat{z}_{\eta}$ in the segment $\left[\hat{y}_{1}, \hat{y}_{2}\right] \subset \Pi_{\rho}^{T}$ which satisfy ${ }^{5}$

$$
\hat{z}_{1}:=\hat{y}_{1}, \quad \hat{z}_{\eta}:=\hat{y}_{2},
$$

\footnotetext{
${ }^{5}$ Since $u$ is differentiable a.e., by Fubini's Theorem, for a.e. $T \in(0, \infty)$ we can find points $\hat{z}_{1}, \ldots, \hat{z}_{\eta}$ such that (3.21) and (3.22) hold. Notice that we do not yet fix the points $\hat{z}_{i}$, since later we will need to impose that they satisfy some additional conditions, see in particular $\left(\pi_{9}\right)$ below.
} 


$$
u \text { is differentiable at } \hat{z}_{i} \quad \forall i=1, \ldots, \eta,
$$

and

$$
\hat{z}_{i} \in\left[\hat{y}_{1}+\frac{i-4 / 3}{2 N}\left(\hat{y}_{2}-\hat{y}_{1}\right), \hat{y}_{1}+\frac{i-2 / 3}{2 N}\left(\hat{y}_{2}-\hat{y}_{1}\right)\right] \quad \forall i=2, \ldots, \eta-1
$$

Notice that

$\left(\pi_{6}\right) \frac{\left|\hat{y}_{2}-\hat{y}_{1}\right|}{6 N} \leq\left|\hat{z}_{i+1}-\hat{z}_{i}\right| \leq \frac{5\left|\hat{y}_{2}-\hat{y}_{1}\right|}{6 N} \quad \forall i=1, \ldots, \eta-1$.

We now fix $T$ to be an arbitrary time in $[\eta+1, \eta+2]$ chosen so that (3.21) and (3.22) hold. Applying Lemma 3.4 (recall also (3.9)), for any $i=1, \ldots, \eta-1$ we can find a time $t_{i} \in$ $[i-1-1 / 8, i-1+1 / 8]$ such that

$$
\bar{\gamma}\left(t_{i}\right) \text { satisfies }(3.8) \quad \forall i=1, \ldots, \eta-1 \text {. }
$$

Given $z \in\left[\hat{y}_{1}, \hat{y}_{2}\right]$ such that $u$ is differentiable at $z$, we can consider the calibrated curve $\gamma_{z}:(-\infty, 0]$ as in (2.6). Notice that, since $\hat{y}_{1}$ and $\hat{y}_{2}$ belong to the projected Aubry set, $u$ is differentiable at $\hat{y}_{1}$ and $\hat{y}_{2}$, so the curves $\gamma_{\hat{y}_{1}}$ and $\gamma_{\hat{y}_{2}}$ are unique and the curves $\gamma_{z}$ cannot intersect $\gamma_{\hat{y}_{1}}$ and $\gamma_{\hat{y}_{2}}$. Hence, provided $r$ is sufficiently small, for any $z \in\left[\hat{y}_{1}, \hat{y}_{2}\right]$ there exist $T_{z} \in[T-1, T+1]$ such that

$$
\gamma_{z}\left(-T_{z}\right) \in \Pi_{\rho}^{0}, \quad \gamma_{z}\left(\left[-T_{z}, 0\right]\right) \subset[0, \eta] \times[-\rho, \rho] .
$$

Recalling (3.21), we now define the following points for all $i=1, \ldots, \eta-1$ (see Figure 1 ), where $\bar{\tau} \in(0,1 / 10)$ is the same as in Proposition 2.7:

$$
\begin{array}{cc}
z_{i,-}:=\gamma_{\hat{z}_{i}}\left(\left[-T_{\hat{z}_{i}}, 0\right]\right) \cap \Pi_{\rho}^{t_{i}}, & z_{i,+}:=\gamma_{\hat{z}_{i}}\left(\left[-T_{\hat{z}_{i}}, 0\right]\right) \cap \Pi_{\rho}^{t_{i}+\bar{\tau}}, \\
z_{i,-}^{\prime}:=\gamma_{\hat{z}_{i+1}}\left(\left[-T_{\hat{z}_{i+1}}, 0\right]\right) \cap \Pi_{\rho}^{t_{i}}, & z_{i,+}^{\prime}:=\gamma_{\hat{z}_{i+1}}\left(\left[-T_{\hat{z}_{i+1}}, 0\right]\right) \cap \Pi_{\rho}^{t_{i}+\bar{\tau}} .
\end{array}
$$

Also, we set

$$
y_{1}^{t}:=\gamma_{\hat{y}_{1}}\left(\left[-T_{y_{1}}, 0\right]\right) \cap \Pi_{\rho}^{t}, \quad y_{2}^{t}:=\gamma_{\hat{y}_{2}}\left(\left[-T_{y_{2}}, 0\right]\right) \cap \Pi_{\rho}^{t} .
$$

By Lemma 3.5(ii), provided $r$ is sufficiently small, $\left(\pi_{6}\right)$ yields

$\left(\pi_{7}\right) \frac{\left|y_{1}^{t_{i}+\bar{\tau}}-y_{2}^{t_{i}+\bar{\tau}}\right|}{7 N} \leq\left|z_{i,+}-z_{i,+}^{\prime}\right| \leq \frac{\left|y_{1}^{t_{i}+\bar{\tau}}-y_{2}^{t_{i}+\bar{\tau}}\right|}{N} \leq \frac{C}{N} \hat{r} \quad \forall i=1, \ldots, \eta-1$.

Also, using again Lemma 3.5(ii), it follows from the construction of $\hat{y}_{1}$ and $\hat{y}_{2}$ (see Lemma 3.7) that, for any $t \in[0, T]$, all points of $\bar{\gamma}\left(\left[0, T_{\hat{r}}\right]\right)$ on $\Pi_{\rho}^{t}$ are at distance at least $\frac{\left|y_{1}^{t}-y_{2}^{t}\right|}{4}$ from $\left\{y_{1}^{t}, y_{2}^{t}\right\}$ (besides the points $\left\{y_{1}^{t}, y_{2}^{t}\right\}$ itself), that is

$\left(\pi_{8}\right) \operatorname{dist}\left(\left(\left(\bar{\gamma}\left(\left[0, T_{\hat{r}}\right]\right) \cap \Pi_{\rho}^{t}\right) \backslash\left\{y_{1}^{t}, y_{2}^{t}\right\}\right),\left\{y_{1}^{t}, y_{2}^{t}\right\}\right) \geq \frac{\left|y_{1}^{t}-y_{2}^{t}\right|}{4} \quad$ for all $t \in[0, T]$.

Since $u$ is differentiable at $z_{i,-}, z_{i,-}^{\prime}, z_{i,+}, z_{i,+}^{\prime}$ (see (3.21) and Proposition 2.4), by Lemma 3.6(ii) and $\left(\pi_{7}\right)$ it follows that (provided $\hat{r}$ is small enough)

$$
\left|\nabla u\left(z_{i,+}\right)-\nabla u\left(z_{i,+}^{\prime}\right)\right| \leq \frac{2 K^{\prime}}{N}\left|y_{1}^{t_{i}+\bar{\tau}}-y_{2}^{t_{i}+\bar{\tau}}\right| .
$$

Hence, since $\mathcal{P}_{0, \bar{\tau}}^{*}\left(z_{i,-}, \nabla u\left(z_{i,-}\right)\right)=\left(z_{i,+}, \nabla u\left(z_{i,+}\right)\right)$ and thanks to $(3.23)$, if $\hat{r}$ is sufficiently small (so that $z_{i,-}$ is close to $\bar{\gamma}\left(t_{i}\right)$ ) we can apply Proposition 2.7 with $x^{0}=z_{i,-}, x^{f}=z_{i,+}^{\prime}$, $p^{0}=\nabla u\left(z_{i,-}\right), p^{f}=\nabla u\left(z_{i,+}^{\prime}\right), r=\left|y_{1}^{t_{i}+\bar{\tau}}-y_{2}^{t_{i}+\bar{\tau}}\right|$, and $\hat{\epsilon}=2\left(1+K^{\prime}\right) / N$ (with $K^{\prime}$ as in Lemma $3.6)$, to find a potential $V_{i}$ which permits to connect $z_{i,-}$ to $z_{i,+}^{\prime}$ on a time interval $\left[\hat{t}_{i}, \hat{t}_{i}+T_{i}^{f}\right]$. Notice that the constant $\sigma=\sigma_{i}$ appearing in the value of the action is an arbitrary number less than $\hat{\epsilon} r^{2}=2\left(1+K^{\prime}\right) \frac{\left|y_{1}^{t_{i}+\bar{\tau}}-y_{2}^{t_{i}+\bar{\tau}}\right|^{2}}{N}$. 


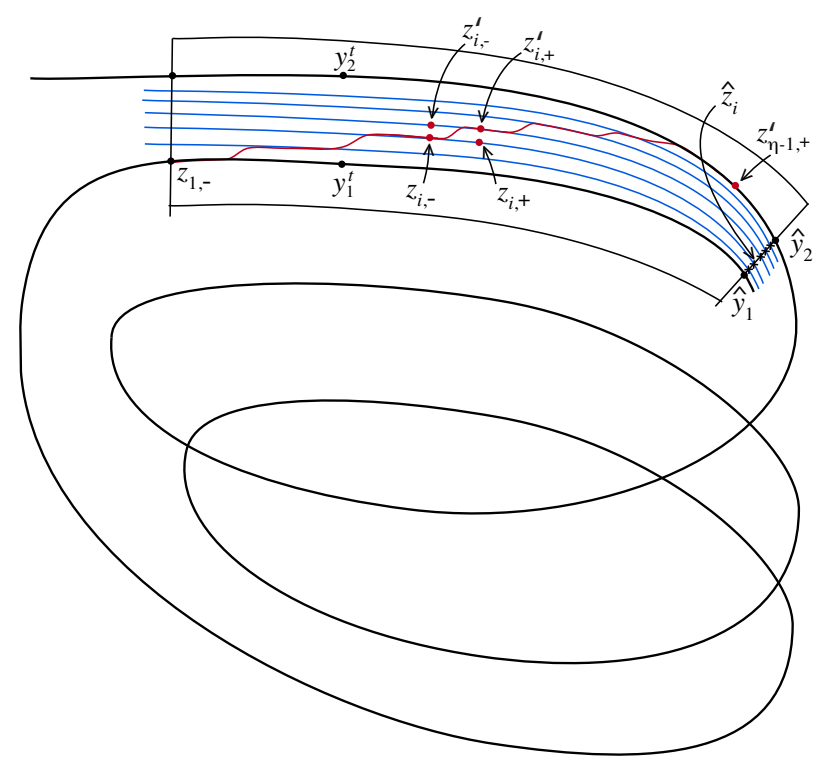

Figure 1: The points $z_{i,-}$ and $z_{i,+}$ (resp. $z_{i,-}^{\prime}$ and $z_{i,+}^{\prime}$ ) are obtained from $z_{i}$ (resp. $z_{i+1}$ ) by intersecting the trajectory passing through $\hat{z}_{i}$ (resp. $\hat{z}_{i+1}$ ) with the hyperplanes $\Pi_{\rho}^{t_{i}}$ and $\Pi_{\rho}^{t_{i}+\bar{\tau}}$. Analogously, the points $y_{1}^{t}$ (resp. $y_{2}^{t}$ ) defined in (3.24) are obtained by intersecting the trajectory passing through $\hat{y}_{1}$ (resp. $\hat{y}_{2}$ ) with the hyperplanes $\Pi_{\rho}^{t}$. Our goal is to connect $z_{i,-}$ to $z_{i,+}^{\prime}$ with a control on the action, in order to obtain a closed curve which satisfies (P2).

We now construct a curve $\gamma:\left[0, T^{\prime}\right] \rightarrow M$ by concatenating $\gamma_{1}:\left[0, T_{1}\right] \rightarrow M$ with $\gamma_{2}$ : $\left[T_{1}, T^{\prime}\right] \rightarrow M$, where

$$
\gamma_{2}(t):=\pi^{*}\left(\phi_{t-T_{1}}^{H}\left(\hat{y}_{2}, \nabla u\left(\hat{y}_{2}\right)\right)\right) \quad \text { connects } z_{\eta-1,+}^{\prime} \text { to } z_{1,-},
$$

while $\gamma_{1}$ is obtained as a concatenation of $2 \eta-1$ pieces: defining by $V:=\sum_{i} V_{i}$ (notice that the support of the $V_{i}$ 's are all disjoint, so the $C^{2}$ norm of $V$ is bounded by $\max _{i}\left\|V_{i}\right\|_{C^{2}}$ ), for every $i=1, \ldots, \eta-1$ we use the flow $(t, z) \mapsto \pi^{*}\left(\phi_{t}^{H+V}(z, \nabla u(z))\right)$ to connect $z_{i,-}$ to $z_{i,+}^{\prime}$ on a time interval $\left[\hat{t}_{i}, \hat{t}_{i}+T_{i}^{f}\right]$, while on $\left[\hat{t}_{i}+T_{i}^{f}, \hat{t}_{i+1}\right](i=1, \ldots, \eta-1)$ we just use the original flow $(t, z) \mapsto \pi^{*}\left(\phi_{t}^{H}(z, \nabla u(z))\right)$ to send $z_{i,+}^{\prime}$ onto $z_{i+1,-}$. (See [19, Subsection 5.3] for more detail.)

In this way we obtain a closed curve $\gamma:\left[0, T^{\prime}\right] \rightarrow M$ (see Figure 1 ) whose action is given by the following formula (see [19, Section 5.4]):

$$
\int_{0}^{T^{\prime}} L_{V}(\gamma(t), \dot{\gamma}(t)) d t=\sum_{i=1}^{\eta-1}\left[\left\langle\nabla u\left(z_{i,+}\right), z_{i,+}^{\prime}-z_{i,+}\right\rangle-\left(u\left(z_{i,+}^{\prime}\right)-u\left(z_{i,+}\right)\right)\right]+\sigma_{i} .
$$

Thanks to $\left(\pi_{7}\right)$ and Lemma 3.6 we deduce that

$$
\left|\left\langle\nabla u\left(z_{i,+}\right), z_{i,+}^{\prime}-z_{i,+}\right\rangle-\left(u\left(z_{i,+}^{\prime}\right)-u\left(z_{i,+}\right)\right)\right| \leq K^{\prime} \frac{\left|y_{1}^{t_{i}+\bar{\tau}}-y_{2}^{t_{i}+\bar{\tau}}\right|^{2}}{N} .
$$

Hence, since $\sigma_{i}$ can be any arbitrary number less than $2\left(1+K^{\prime}\right) \frac{\left|y_{1}^{t_{i}+\bar{\tau}}-y_{2}^{t_{i}+\bar{\tau}}\right|^{2}}{N}$, we can choose

$$
\sigma_{i}:=\left(u\left(z_{i,+}^{\prime}\right)-u\left(z_{i,+}\right)\right)-\left\langle\nabla u\left(z_{i,+}\right), z_{i,+}^{\prime}-z_{i,+}\right\rangle
$$

to enforce

$$
\int_{0}^{T^{\prime}} L_{V}(\gamma(t), \dot{\gamma}(t)) d t=0
$$

as desired. This concludes the proof of (P2). 


\subsubsection{A "good" critical subsolution for $H$}

To prove (P1), we first need to construct a $C^{1,1}$ critical subsolution $v$ which is " $C^{2}$ in average". Recall that, for every $t>0$, the function $h_{t}: M \times M \rightarrow \mathbb{R}$ is defined by

$$
h_{t}(x, y):=\inf \int_{0}^{t} L(\gamma(s), \dot{\gamma}(s)) d s,
$$

where the infimum is taken over all Lipschitz curves $\gamma:[0, t] \rightarrow M$ such that $\gamma(0)=x$ and $\gamma(t)=y$.

Lemma 3.8. Let $\Psi$ be as in $\left(\pi_{3}\right)-\left(\pi_{5}\right)$, and let $y_{1}^{t}=\left(t, \ell_{1}^{t}\right), y_{2}^{t}=\left(t, \ell_{2}^{t}\right) \in \mathscr{O} \cap \Pi_{\rho}^{t}$ be as in (3.24). There exists $s_{0}>0$ small but universal such that the critical subsolution $v: M \rightarrow \mathbb{R}$ defined by

$$
v(x):=\mathcal{T}_{s_{0}}^{+} u(x)=\sup _{y \in M}\left\{u(y)-h_{s_{0}}(x, y)\right\} \quad \forall x \in M,
$$

is universally $C^{1,1}$ and, for a.e. $t \in[0, T]$, it satisfies ${ }^{6}$

$$
\int_{0}^{1}\left|\left\langle\nabla^{2} v\left(y_{1}^{t}+s\left(y_{2}^{t}-y_{1}^{t}\right)\right) \cdot e_{2}, e_{2}\right\rangle-\left\langle L_{t} \cdot e_{2}, e_{2}\right\rangle\right| d s \leq \omega\left(\left|\ell_{1}^{t}\right|+\left|\ell_{2}^{t}\right|\right)
$$

for some universal modulus of continuity $\omega: \mathbb{R}^{+} \rightarrow \mathbb{R}^{+}$.

Proof. The fact that $v$ is a critical subsolution is standard, see for instance [7].

By semiconcavity, there is a bounded family of $C^{2}\left(\mathcal{U}^{\prime}, \mathbb{R}\right)$ such that

$$
u=\inf _{f \in \mathcal{F}}\{f\} .
$$

Moreover, thanks to the estimate (3.13) on $D^{2} u$ provided by the Green bundles, we may assume that

$$
\nabla^{2} f(x) \leq L_{t}+\omega^{\prime}(r) \mathrm{Id} \quad \forall x \in \Pi_{r}^{t}, r \in(0, \rho], t \in[0, T], f \in \mathcal{F} .
$$

Then, for every $s_{0}>0$ we have

$$
v=\inf _{f \in \mathcal{F}}\left\{T_{s_{0}}^{+} f\right\} .
$$

By [7] it is known that, for $s_{0}>0$ small enough, $v \in C^{1,1}, v=u$, and $\nabla v=\nabla u$ on the projected Aubry set. Since $\nabla v=\nabla u$ on $\mathscr{O}$, by (3.14) we get

$$
\nabla v\left(y_{2}^{t}\right)=\nabla v\left(y_{1}^{t}\right)+L_{t} \cdot\left(y_{2}^{t}-y_{1}^{t}\right)+\omega\left(\left|\ell_{1}^{t}\right|+\left|\ell_{2}^{t}\right|\right)\left|y_{1}^{t}-y_{2}^{t}\right| \quad \forall t \in[0, T] .
$$

Since $y_{2}^{t}-y_{1}^{t}$ is parallel to $e_{2}$, rewriting the above expression using the fundamental theorem of calculus (recall that $v \in C^{1,1}$ ) we have

$$
\int_{0}^{1}\left\langle\nabla^{2} v\left(y_{1}^{t}+s\left(y_{2}^{t}-y_{1}^{t}\right)\right) \cdot e_{2}, e_{2}\right\rangle d s \leq\left\langle L_{t} \cdot e_{2}, e_{2}\right\rangle+\omega\left(\left|\ell_{1}^{t}\right|+\left|\ell_{2}^{t}\right|\right) \quad \text { for a.e. } t \in[0, T],
$$

where $\nabla^{2} v$ is the pointwise Hessian of $v$.

By [7, Lemma 3] there is $s_{0}>0$ such that, for every $s \in\left[0, s_{0}\right], \mathcal{T}_{s}^{+}(\mathcal{F})$ is a bounded set in $C^{2}(M, \mathbb{R})$. Since the Hessian of a $C^{2}$ function $f$ is transported by the linearized Hamiltonian flow along the calibrating trajectories (see for instance the discussion in [19] after Lemma 5.3), and since all the trajectories are close (as a function of $r$ ) to the trajectory passing through $t e_{1}$ (see (3.11)), we deduce from (3.25) that, for all $r \in(0, \rho]$,

$$
\nabla^{2} v \leq L_{t}+\omega(r) \text { Id } \quad \text { a.e. on } \Pi_{r}^{t} \text {, for a.e. } t \in[0, T] .
$$

\footnotetext{
${ }^{6}$ Notice that, being $C^{1,1}, v$ is twice differentiable a.e.
} 
Hence, combining this bound with (3.26) we get

$$
\int_{0}^{1}\left|\left\langle\left[\nabla^{2} v\left(y_{1}^{t}+s\left(y_{2}^{t}-y_{1}^{t}\right)\right)-L_{t}\right] \cdot e_{2}, e_{2}\right\rangle\right| d s \leq \omega\left(\left|\ell_{1}^{t}\right|+\left|\ell_{2}^{t}\right|\right),
$$

as desired.

Combining Lemmas 3.8 and 2.20, we can also prove that there are many points where $v$ is " $C^{2}$ in average".

Lemma 3.9. With the same notation as in Lemma 3.8, let $y_{1}^{t}, y_{2}^{t} \in \mathscr{O} \cap \Pi_{r}^{t}, r:=\left|\ell_{1}^{t}\right|+\left|\ell_{2}^{t}\right| \in$ $(0, \rho]$. Then, for a.e. $t \in[0, T]$ there exists a set $A_{t} \subset\left[y_{1}^{t}, y_{2}^{t}\right]$ such that

$$
\left|A_{t}\right| \geq(1-\sqrt{\omega(r)})\left|y_{1}^{t}-y_{2}^{t}\right|
$$

and

$$
\frac{1}{R} \int_{0}^{R}\left|\left\langle\nabla^{2} v\left(z+s e_{2}\right) \cdot e_{2}, e_{2}\right\rangle-\left\langle L_{t} \cdot e_{2}, e_{2}\right\rangle\right| d s \leq C_{1} \sqrt{\omega(r)}
$$

for all $z \in A_{t}$ and $R \in\left[-\left|y_{1}^{t}-z\right|,\left|y_{2}^{t}-z\right|\right]$.

Proof. We simply apply Lemma 2.20 to the one dimensional function

$$
f(s):=\left|\left\langle\nabla^{2} v\left(z+s e_{2}\right) \cdot e_{2}, e_{2}\right\rangle-\left\langle L_{t} \cdot e_{2}, e_{2}\right\rangle\right| \chi_{\left[-\left|y_{1}-z\right|,\left|y_{2}-z\right|\right]}(s)
$$

with $\delta=\sqrt{\omega(r)}$, and use Lemma 3.8.

Thanks to Lemma 3.9 above, we see that, provided $r$ is sufficiently small and (if necessary) by slightly changing the system of coordinates (so the the above lemmas apply with $t=t_{i}$ for all $i=1, \ldots, \eta-1)$, the points $\hat{z}_{1}, \ldots, \hat{z}_{\eta}$ can be chosen so such that

$\left(\pi_{9}\right) z_{i,-}, z_{i,-}^{\prime} \in A_{t_{i}}$ for all $i=1, \ldots, \eta-1$.

\subsubsection{Construction of a global critical subsolution}

As before, we will denote by $\omega: \mathbb{R}^{+} \rightarrow \mathbb{R}^{+}$a modulus of continuity which may change from line to line.

Our goal is to construct a critical subsolution $v_{V}: M \rightarrow \mathbb{R}$ satisfying (P1). We proceed as follows: first, for any $i=1, \ldots, t_{i}$ we define $u_{0}^{i}$ and $u_{V}^{i}$ as the $C^{1,1}$ solutions of the Dirichlet problems

$$
\begin{aligned}
& \left\{\begin{array}{l}
H\left(z, \nabla u_{0}^{i}(z)\right)=0 \\
u_{0}^{i}=v \text { on } \Pi_{\rho}^{t_{i},},
\end{array} \text { in }\left[t_{i}, t_{i}+3 \bar{\tau}\right] \times \Pi_{\rho}^{t_{i},},\right. \\
& \left\{\begin{array}{l}
H_{V}\left(z, \nabla u_{V}^{i}(z)\right)=0 \\
u_{V}^{i}=v \text { on } \Pi_{\rho}^{t_{i}},
\end{array}\right.
\end{aligned}
$$

where $v$ is as in Lemma 3.8 (see Lemma 2.6(iv)). Let $\gamma:\left[0, T^{\prime}\right] \rightarrow M$ be the closed trajectory constructed in Section 3.3.2, and define $\Gamma_{i}:=\gamma\left(\left[\hat{t}_{i}+T_{i}^{f}, \hat{t}_{i+1}\right]\right)$ to be the piece of curve which connects $z_{i,+}^{\prime}$ to $z_{i+1,-}$ (see Figure 2). In complete analogy with [19, Section 5.5, Property $(\pi 3)]$, we have

$$
u_{0}^{i}=u_{V}^{i}, \quad \nabla u_{0}^{i}=\nabla u_{V}^{i} \quad \text { on } \Gamma_{i} \cap \mathcal{C}_{i}, \text { where } \quad \mathcal{C}_{i}:=\bigcup_{t \in\left[t_{i}, t_{i}+3 \bar{\tau}\right]}\left[y_{1}^{t}, y_{2}^{t}\right] .
$$

Also, because $y_{1}^{t}$ and $y_{2}^{t}$ are in the projected Aubry set,

$$
u_{0}^{i}=u_{V}^{i}=v, \quad \nabla u_{0}^{i}=\nabla u_{V}^{i}=\nabla v \quad \text { on } \partial_{\text {lat }} \mathcal{C}_{i}, \text { where } \quad \partial_{\text {lat }} \mathcal{C}_{i}:=\bigcup_{t \in\left[t_{i}, t_{i}+3 \bar{\tau}\right]}\left\{y_{1}^{t}, y_{2}^{t}\right\} .
$$


We claim that

$$
\left|u_{0}^{i}(x)-u_{V}^{i}(x)\right| \leq \omega(\hat{r}+\epsilon) \operatorname{dist}\left(x, \Gamma_{i}\right)^{2} \quad \forall x \in \mathcal{C}_{i} .
$$

Indeed, since by $\left(\pi_{1}\right),\left(\pi_{9}\right)$, and Lemmas 3.8 and 3.9

$$
\frac{1}{R} \int_{0}^{R}\left|\left\langle\nabla^{2} v\left(z+s e_{2}\right) \cdot e_{2}, e_{2}\right\rangle-\left\langle L_{t_{i}} \cdot e_{2}, e_{2}\right\rangle\right| d s \leq C_{1} \sqrt{\omega\left(\left|\ell_{1}^{t_{i}}\right|+\left|\ell_{2}^{t_{i}}\right|\right)}
$$

for $z=z_{i,-}, z_{i,-}^{\prime}$ and $R \in\left[-\left|y_{1}^{t_{i}}-z\right|,\left|y_{2}^{t_{i}}-z\right|\right]$, and the flow of the vector field $\frac{\partial H}{\partial p}(x, \nabla v(x))$ is bi-Lipschitz (since $v$ is $C^{1,1}$ ), we deduce that (recall that the Hessian of a solution is propagated along the linearized flow)

$$
\frac{1}{R} \int_{0}^{R}\left|\left\langle\nabla^{2} u_{0}^{i}\left(z_{i}^{t}+s e_{2}\right) \cdot e_{2}, e_{2}\right\rangle-\left\langle L_{t} \cdot e_{2}, e_{2}\right\rangle\right| d s \leq C \sqrt{\omega\left(\left|\ell_{1}^{t_{i}}\right|+\left|\ell_{2}^{t_{i}}\right|\right)}
$$

for all $R \in\left[-\left|y_{1}^{t}-z\right|,\left|y_{2}^{t}-z\right|\right]$ and $t \in\left[t_{i}+2 \bar{\tau}, t_{i}+3 \bar{\tau}\right]$, where

$$
z_{i}^{t}:=\gamma_{\hat{z}_{i+1}}\left(\left[-T_{\hat{z}_{i+1}}, 0\right]\right) \cap \Pi_{\rho}^{t} .
$$

Also, because $\|V\|_{C^{2}} \leq \epsilon$, the linearized flows of $H$ and $H_{V}$ are close in terms of $\epsilon$ (see [19, Proof of Lemma 5.5]), hence

$$
\frac{1}{R} \int_{0}^{R}\left|\left\langle\nabla^{2} u_{V}^{i}\left(z_{i}^{t}+s e_{2}\right) \cdot e_{2}, e_{2}\right\rangle-\left\langle L_{0}^{t} \cdot e_{2}, e_{2}\right\rangle\right| d s \leq C \sqrt{\omega\left(\left|\ell_{1}^{t_{i}}\right|+\left|\ell_{2}^{t_{i}}\right|\right)}+\omega(\epsilon)
$$

for all $R \in\left[-\left|y_{1}^{t}-z\right|,\left|y_{2}^{t}-z\right|\right]$ and $t \in\left[t_{i}+2 \bar{\tau}, t_{i}+3 \bar{\tau}\right]$. Since $\left|\ell_{1}^{t_{i}}\right|+\left|\ell_{2}^{t_{i}}\right| \leq C \hat{r}$ (because $\hat{y}_{1}, \hat{y}_{2} \in \Pi_{\hat{r}}^{T}$ and the flow is Lipschitz on the Aubry set), this estimate combined with (3.27) and a simple Taylor expansion proves (3.29).

We consider now $\Theta_{i}: \mathbb{R} \rightarrow[0,1]$ a smooth function such that

$$
\begin{cases}\Theta_{i}(z)=\Theta_{i}\left(z_{1}\right)=1 & \text { if } z_{1} \in\left[t_{i}, t_{i}+3 \bar{\tau} / 2\right], \\ \Theta_{i}(z)=\Theta_{i}\left(z_{1}\right)=0 & \text { if } z_{1} \in\left[t_{i}+5 \bar{\tau} / 2, t_{i}+3 \bar{\tau}\right],\end{cases}
$$

and define $\tilde{u}_{i}$ as

$$
\tilde{u}_{i}(z):= \begin{cases}\Theta_{i}(z) u_{V}^{i}(z)+\left(1-\Theta_{i}(z)\right) v(z) & \text { for } z \in \mathcal{C}_{i}, \\ v(z) & \text { for } z \in \mathcal{C}_{i}^{\prime} \backslash \mathcal{C}_{i},\end{cases}
$$

where

$$
\mathcal{C}_{i}^{\prime}:=\bigcup_{t \in\left[t_{i}, t_{i}+3 \bar{\tau}\right]}\left[y_{1}^{t,}, y_{2}^{t,{ }^{\prime}}\right], \quad y_{1}^{t,{ }^{\prime}}:=y_{1}^{t}-\frac{y_{2}^{t}-y_{1}^{t}}{4}, \quad y_{2}^{t,{ }^{\prime}}:=y_{2}^{t}+\frac{y_{2}^{t}-y_{1}^{t}}{4}
$$

(see Figure 2).

Thanks to (3.28), $\tilde{u}_{i}$ is of class $C^{1,1}$ inside $\mathcal{C}_{i}^{\prime}$. Moreover, for every $z \in \mathcal{C}_{i}$ we have

$$
\nabla \tilde{u}_{i}(z)=\left(u_{V}^{i}(z)-v(z)\right) \nabla \Theta_{i}(z)+\Theta_{i}(z) \nabla u_{V}^{i}(z)+\left(1-\Theta_{i}(z)\right) \nabla v(z) .
$$

Set

$$
P_{i}(z):=\Theta_{i}(z) \nabla u_{V}^{i}(z)+\left(1-\Theta_{i}(z)\right) \nabla v(z) \quad \forall z \in \mathcal{C}_{i} .
$$

By convexity of $H$ in the $p$ variable we get

$$
H_{V}\left(z, P_{i}(z)\right) \leq 0 \quad \forall z \in \mathcal{C}_{i} .
$$

Moreover, since $v$ is a subsolution for $H$,

$$
H_{V}\left(z, \nabla \tilde{u}_{i}(z)\right) \leq 0 \quad \forall z \in \mathcal{C}_{i}^{\prime} \backslash \mathcal{C}_{i}, \quad u_{0}^{i}(z)-v(z) \geq 0 \quad \forall z \in \mathcal{C}_{i} .
$$




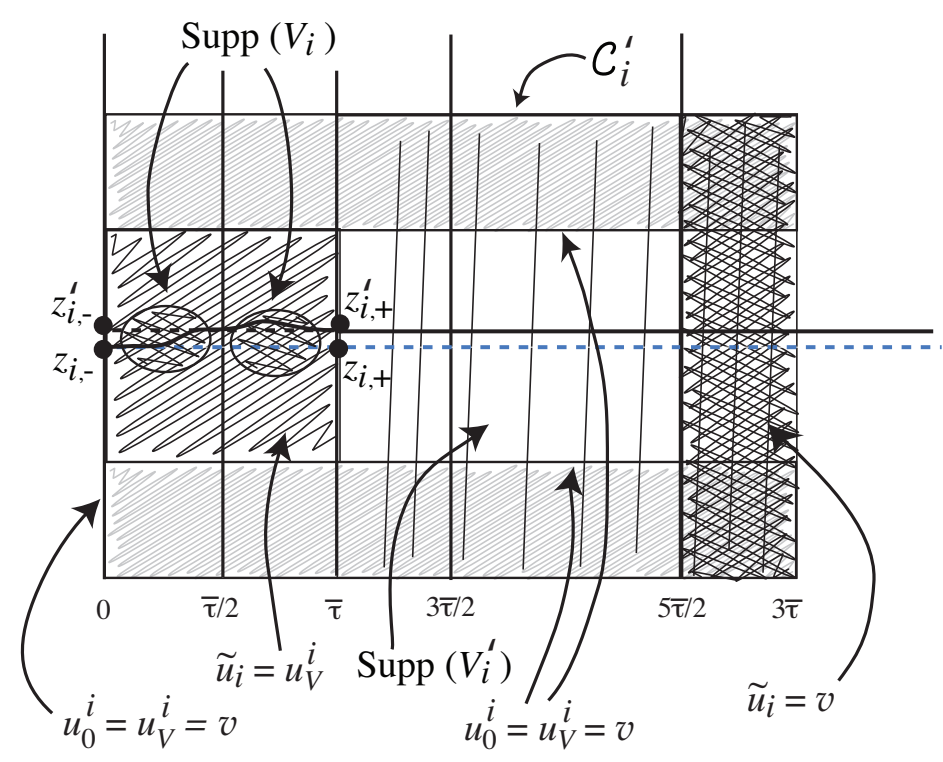

Figure 2: The curve $\Gamma_{i}$ corresponds to the horizontal segment starting from the point $z_{i,+}^{\prime}$ and going to the right. The function $\tilde{u}_{i}$ is obtained by interpolating (using a cut-off function) between $u_{V}^{i}$ (the viscosity solution for $H_{V}$ ) and $v$ (the critical subsolution for $H$ constructed in Section 3.3.3) inside the "cylinder" $\mathcal{C}_{i}^{\prime}$. Then, by adding a new potential $V_{i}^{\prime}$, small in $C^{2}$ topology and supported inside $\mathcal{C}_{i}^{\prime} \cap\left\{z=\left(z_{1}, \hat{z}\right) \mid z_{1} \in[\bar{\tau}, 3 \bar{\tau}]\right\}$, we can ensure that $H_{V_{i}+V_{i}^{\prime}}\left(z, \nabla \tilde{u}_{i}(z)\right) \leq 0$. Since the cylinders $\mathcal{C}_{i}^{\prime}$ are disjoint, we can repeat this construction for $i=1, \ldots, \eta-1$ to find a global critical subsolution $\tilde{u}$ and a potential $\tilde{V}$ so that (P1) and (P2) hold.

Also, since $\nabla \Theta_{i}$ points in the direction of $-e_{1}$, by (3.11) we get

$$
\left\langle\frac{\partial H}{\partial p}\left(z, P_{i}(z)\right), \nabla \Theta_{i}(z)\right\rangle \leq-\frac{1}{2}\left|\nabla \Theta_{i}(z)\right| \quad \forall z \in \mathcal{C}_{i} .
$$

Then, using that $u_{0}^{i} \geq v$ and Taylor's formula, we obtain

$$
\begin{aligned}
H_{V}\left(z, \nabla \tilde{u}_{i}(z)\right) \leq & H_{V}\left(z, P_{i}(z)\right)+\left(u_{V}^{i}(z)-v(z)\right)\left\langle\frac{\partial H}{\partial p}\left(z, P_{i}(z)\right), \nabla \Theta_{i}(z)\right\rangle \\
& +K\left|\nabla \Theta_{i}(z)\right|^{2}\left|u_{V}^{i}(z)-v(z)\right|^{2} \\
\leq & \left(u_{V}^{i}(z)-u_{0}^{i}(z)\right)\left\langle\frac{\partial H}{\partial p}\left(z, P_{i}(z)\right), \nabla \Theta_{i}(z)\right\rangle-\frac{1}{2}\left|u_{0}^{i}(z)-v(z)\right|\left|\nabla \Theta_{i}(z)\right| \\
& +2 K\left|\nabla \Theta_{i}(z)\right|^{2}\left|u_{V}^{i}(z)-u_{0}^{i}(z)\right|^{2}+2 K\left|\nabla \Theta_{i}(z)\right|^{2}\left|u_{0}^{i}(z)-v(z)\right|^{2} \\
\leq & C\left|\nabla \Theta_{i}(z)\right|\left|u_{V}^{i}(z)-u_{0}^{i}(z)\right|,
\end{aligned}
$$

where we used that $\left|u_{V}^{i}-u_{0}^{i}\right|$ and $\left|u_{0}^{i}-v\right|$ are small to absorb the quadratic terms into the linear ones. Since the last term in the above equation is of order $\omega(\hat{r}+\epsilon) \operatorname{dist}\left(x, \Gamma_{i}\right)^{2}$ (by (3.29)) and vanishes both outside $\mathcal{C}_{i}$ and outside the support of $\Theta$, we deduce that

$$
H_{V}\left(z, \nabla \tilde{u}_{i}(z)\right) \leq 0
$$

inside $\mathcal{C}_{i}^{\prime} \cap\left(\left[t_{i}, t_{i}+3 \bar{\tau} / 2\right] \cup\left[t_{i}+5 \bar{\tau} / 2, t_{i}+3 \bar{\tau}\right]\right)$, and (using $\left.\left(\pi_{8}\right)\right)$

$$
H_{V}\left(z, \nabla \tilde{u}_{i}(z)\right) \leq \omega(\hat{r}+\epsilon) \min \left\{\operatorname{dist}\left(x, \Gamma_{i}\right)^{2}, \operatorname{dist}\left(x, \partial_{l a t} \mathcal{C}_{i}^{\prime}\right)^{2}\right\}
$$


inside $\mathcal{C}_{i}^{\prime} \cap\left[t_{i}+3 \bar{\tau} / 2, t_{i}+5 \bar{\tau} / 2\right]$, where

$$
\partial_{l a t} \mathcal{C}_{i}^{\prime}:=\bigcup_{t \in\left[t_{i}+2 \bar{\tau}, t_{i}+3 \bar{\tau}\right]}\left\{y_{1}^{t,,^{\prime}}, y_{2}^{t, \prime^{\prime}}\right\}
$$

By choosing $\hat{r}$ and $\epsilon$ sufficiently small, it is easy to see that we can add a potential $V_{i}^{\prime} \leq 0$, small in $C^{2}$ topology, which vanishes on $\Gamma$ and supported inside $\mathcal{C}_{i}^{\prime}$, so that

$$
H_{V+V_{i}^{\prime}}\left(z, \nabla \tilde{u}_{i}(z)\right) \leq 0 \quad \text { in } \mathcal{C}_{i}^{\prime} .
$$

Then the function $\tilde{u}$ obtained by gluing together the functions $\tilde{u}_{i}$ with $v$ is a global critical subsolution for $H_{\tilde{V}}$ with $\tilde{V}:=V+\sum_{i} V_{i}^{\prime}$ (notice that the support of the $V_{i}^{\prime}$ 's are all disjoint, so the $C^{2}$ norm of $\sum_{i} V_{i}^{\prime}$ is bounded by $\max _{i}\left\|V_{i}^{\prime}\right\|_{C^{2}}$ ), yielding (P1) and concluding the proof of Proposition 3.3.

\section{Examples}

Recall that the minimal set of a Lipschitz vector field on a surface is called exceptional (or minimal) if it is neither a fixed point, nor a closed trajectory, nor the whole surface (see [28]). By the Poincaré-Bendixon Theorem, exceptional minimal sets do not exist on the twodimensional sphere. The purpose of this section is to construct Tonelli Hamiltonians with exceptional minimal Aubry sets on orientable surfaces with positive genus.

\subsection{Preliminaries on the Mather functions}

Let $M$ be a smooth compact Riemannian manifold without boundary of dimension $n \geq 2$ and $H: T^{*} M \rightarrow \mathbb{R}$ a Tonelli Hamiltonian of class $C^{2}$. Denote by $L: T M \rightarrow \mathbb{R}$ the Tonelli Lagrangian of class $C^{2}$ associated with $H$ by Legendre-Fenchel duality (see Section 2.1). The flow $\phi_{t}^{L}$ of $L$ is conjugated with the Hamiltonian flow through the Legendre transform $\mathcal{L}$ : $T^{*} M \rightarrow T M$ defined by $\mathcal{L}(x, p):=\left(x, \frac{\partial H}{\partial p}(x, p)\right)$, that is

$$
\phi_{t}^{L}=\mathcal{L} \circ \phi_{t}^{H} \circ \mathcal{L}^{-1} .
$$

Denote by $\mathcal{M}(L)$ the set of probability measures on $T M$ which are invariant under the Lagrangian flow. Recall that the homology $\rho(\mu) \in H_{1}(M, \mathbb{R})=H^{1}(M, \mathbb{R})^{*}$ of a measure $\mu \in \mathcal{M}(L)$ is determined by

$$
\langle\rho(\mu),[\omega]\rangle=\int_{T M} \omega_{x}(v) d \mu(x, v),
$$

where $\omega$ is any closed 1 -form on $M$ and $[\omega] \in H^{1}(M, \mathbb{R})$ is its cohomology class. The action of $\mu$ with respect to $L$ is defined as

$$
\mathbb{A}_{L}(\mu):=\int_{T M} L d \mu
$$

The Mañé critical value of $L$ and $H$ can be recovered as

$$
\mathbf{c}[H]=\mathbf{c}[L]:=-\min \left\{\mathbb{A}_{L}(\mu) \mid \mu \in \mathcal{M}(L)\right\} .
$$

The Mather $\alpha$ and $\beta$ functions associated with $L$ (or equivalently with $H$ ),

$$
\alpha_{L}: H^{1}(M, \mathbb{R}) \longrightarrow \mathbb{R} \quad \beta_{L}: H_{1}(M, \mathbb{R}) \longrightarrow \mathbb{R},
$$

are defined as

$$
\beta_{L}(h):=\min \left\{\mathbb{A}_{L}(\mu) \mid \mu \in \mathcal{M}(L), \rho(\mu)=h\right\} \quad \forall h \in H_{1}(M, \mathbb{R})
$$


and

$$
\alpha_{L}([\omega]):=\mathbf{c}[L-\omega] \quad \forall[\omega] \in H^{1}(M, \mathbb{R}) .
$$

They are convex functions with superlinear growth which are conjugate (see [26, Theorem 1]), that is

$$
\alpha_{L}(c)=\max \left\{\langle h, c\rangle-\beta_{L}(h) \mid h \in H_{1}(M, \mathbb{R})\right\} \quad \forall c \in H^{1}(M, \mathbb{R})
$$

and

$$
\beta_{L}(h)=\max \left\{\langle h, c\rangle-\alpha_{L}(c) \mid c \in H^{1}(M, \mathbb{R})\right\} \quad \forall h \in H_{1}(M, \mathbb{R}) .
$$

Let us now introduce some definitions and notation. We call flat of $\beta_{L}$ any non-trivial maximal convex domain in $H_{1}(M, \mathbb{R})$ on which $\beta_{L}$ is an affine function. Moreover we say that a flat is radial if it is contained in a set of the form $\langle h\rangle=\{t h \mid t \in \mathbb{R}\}$ with $h \in H_{1}(M, \mathbb{R})$. By conjugation, any flat $F$ of $\beta_{L}$ is associated with a non-differentiability point of $\alpha_{L}$ : more precisely, if $c \in H^{1}(M, \mathbb{R})$ satisfies

$$
\alpha_{L}(c)=\langle h, c\rangle-\beta_{L}(h) \quad \forall h \in F,
$$

then all affine functions $c^{\prime} \mapsto\left\langle h, c^{\prime}\right\rangle-\beta_{L}(h)$ with slope $h \in F$ are supporting functions for $\alpha$ at c. Given $h \in H_{1}(M, \mathbb{R})$ and $c=[\omega] \in H^{1}(M, \mathbb{R})$, let

$$
\begin{aligned}
\mathcal{M}_{h}(L):=\arg \min \left\{\mathbb{A}_{L}(\mu) \mid \mu \in \mathcal{M}(L), \rho(\mu)=h\right\}, \\
\mathcal{M}^{c}(L)=\mathcal{M}^{\omega}(L):=\arg \min \left\{\mathbb{A}_{L-\omega}(\mu) \mid \mu \in \mathcal{M}(L)\right\} .
\end{aligned}
$$

Note that, by the above properties, for every $c \in H^{1}(M, \mathbb{R})$ we have

$$
\rho\left(\mathcal{M}^{c}(L)\right)=\left\{h \in H_{1}(M, R) \mid \alpha_{L}(c)+\beta_{L}(h)=\langle h, c\rangle\right\} .
$$

Finally, we recall that a homology class is rational if there is $t \in \mathbb{R}$ such that $t h \in H_{1}(M, \mathbb{Z})$.

\subsection{Exceptional minimal hyperbolic Aubry sets on the 2-torus}

Let $M$ be a torus of dimension 2 and fix $P$ a point in $M$. The open manifold $M \backslash\{P\}$ can be equipped with a hyperbolic metric of curvature -1 . Let us fix a simple close curve $\chi$ with length $\ell>0$ which bounds a small open disc $D$ containing $P$, and another simple close curve $\chi^{\prime}$ with length $\ell^{\prime} \in(0, \ell)$ which is contained in $D$ and which bounds a small open disc $D^{\prime}$ containing $P$. We can choose $\chi^{\prime}$ so small that $d\left(\chi, \chi^{\prime}\right)>\ell$, where $d$ denotes the distance with respect to the hyperbolic metric. We now change the hyperbolic metric on $D^{\prime} \backslash\{P\}$ into a smooth metric on $D^{\prime}$ which coincide with the former metric on the boundary of $D^{\prime}$. In this way we obtain a smooth metric $g$ on $M$. We will be concerned with the geodesic Lagrangian $L: T M \rightarrow \mathbb{R}$ defined as

$$
L(x, v):=\frac{1}{2}\|v\|_{x}^{2} \quad \forall(x, v) \in T M,
$$

and we denote by $H$ the associated Hamiltonian. We notice that, for every $c=[\omega] \in H^{1}(M, \mathbb{R})$, $\mathbf{c}[L-\omega] \geq 0$ and $\mathbf{c}[L-\omega]=0 \Leftrightarrow[\omega]=0$. For every $c=[\omega] \in H^{1}(M, \mathbb{R})$ we denote respectively by $\tilde{\mathcal{A}}(c)$ and $\mathcal{A}(c)$ the Aubry set and projected Aubry set of the Hamiltonian associated with the Lagrangian $L-\omega$, that is, of the Hamiltonian given by $H(x, p)=\frac{1}{2}\left\|p+\omega_{x}\right\|^{2}$, where $\|\cdot\|$ denotes the cometric on $T^{*} M$. In the sequel, by abuse of notation, we will look at the Aubry set as a subset of $T M$ via the identification between $T M$ and $T^{*} M$ given by the Legendre transform.

Lemma 4.1. For every closed form $\omega$ with $c=[\omega] \neq 0$, we have

$$
\mathcal{A}(c) \cap D^{\prime}=\emptyset \text {. }
$$


Proof. Let us first show that $\mathcal{A}(c)$ cannot be included in $D$. Argue by contradiction and pick a positively recurrent point $\theta=(x, p)$ of the Aubry set $\tilde{\mathcal{A}}(c)$. Let $\gamma(t)=\pi^{*}\left(\phi_{t}^{H}(x, p)\right)$ for $t \in \mathbb{R}$. Then there exists a sequence $t_{k} \rightarrow+\infty$ such that $\theta=\lim _{k \rightarrow \infty} \phi_{t_{k}}^{H}(\theta)$. Since $\theta$ belongs to the Aubry set, the curve $\gamma$ is calibrated, that is, for every critical solution $u: M \rightarrow \mathbb{R}$ we have

$$
0=\lim _{k \rightarrow \infty} u\left(\gamma\left(t_{k}\right)\right)-u(x)=\lim _{k \rightarrow \infty} \int_{0}^{t_{k}}\left[L(\gamma(t), \dot{\gamma}(t))-\omega_{\gamma(t)}(\dot{\gamma}(t))\right] d t+\mathbf{c}[L-\omega] t_{k} .
$$

Let $f: D \rightarrow \mathbb{R}$ be a smooth function such that $d f=\omega$ on $D$. Since $\gamma([0, \infty)) \subset \mathcal{A}(c) \subset D$ by assumption, we have

$$
\int_{0}^{t_{k}} \omega_{\gamma(t)}(\dot{\gamma}(t)) d t=f\left(\gamma\left(t_{k}\right)\right)-f(\gamma(0))
$$

Hence, since $L \geq 0$, combining the two estimates above and letting $k \rightarrow \infty$ we obtain $\mathbf{c}[L-\omega] \leq$ 0 . Recalling that $\mathbf{c}[L-\omega] \geq 0$ and $\mathbf{c}[L-\omega]=0 \Leftrightarrow[\omega]=0$, we infer that $\mathbf{c}[L-\omega]=0$ which means that $\omega$ is exact, a contradiction.

Assume now that $\mathcal{A}(c)$ intersects both $D^{\prime}$ and $M \backslash D$. Then there are a calibrated curve $\gamma: \mathbb{R} \rightarrow \mathcal{A}(c)$ and $T_{1}$ such that $\gamma(0) \in \partial D^{\prime}$ and $\gamma\left(T_{1}\right) \in \partial D$. The $\alpha$-limit and $\omega$-limit sets of $\gamma$ contain positively recurrent points, so (by the previous argument) they cannot be contained in $D$. Therefore we may assume that $T_{1}<0$ and that there is $T_{2}>0$ such that $\gamma\left(T_{2}\right) \in \partial D$ and $\gamma\left(\left(T_{1}, T_{2}\right)\right) \subset D$. Let $\bar{\chi}:\left[T_{1}, T_{2}\right] \rightarrow \partial D$ be a smooth constant-speed curve corresponding to piece of the curve $\chi$ joining $\gamma\left(T_{1}\right)$ to $\gamma\left(T_{2}\right)$ with constant speed. Since $d\left(\chi, \chi^{\prime}\right)>\ell$ and while the length of $\chi$ is $\ell$

$$
\int_{T_{1}}^{T_{2}}\|\dot{\gamma}(t)\|_{\gamma(t)}^{2} d t>\frac{\ell^{2}}{T_{2}-T_{1}} \geq \int_{T_{1}}^{T_{2}}\|\dot{\bar{\chi}}(t)\|_{\bar{\chi}(t)}^{2} d t
$$

which shows that (since both curves are contained in $\bar{D}$ the integral of $\omega$ along them just depends on their end-points)

$$
\int_{T_{1}}^{T_{2}}\left[\|\dot{\gamma}(t)\|_{\gamma(t)}^{2}-\omega_{\gamma(t)}(\dot{\gamma}(t))\right] d t>\frac{\ell^{2}}{T_{2}-T_{1}} \geq \int_{T_{1}}^{T_{2}}\left[\|\dot{\bar{\chi}}(t)\|_{\bar{\chi}(t)}^{2}-\omega_{\bar{\chi}(t)}(\dot{\bar{\chi}}(t))\right] d t .
$$

This contradicts the minimality of $\gamma$ (see (2.2) and (2.9)), proving the result.

Lemma 4.2. The function $\beta_{L}$ has no flat.

Proof. By homogeneity of $L$, the function $\beta_{L}$ is quadratic in the radial direction, that is $\beta(t h)=$ $t^{2} \beta(h)$ for any $h \in h_{1}(M, \mathbb{R})$ and $t \geq 0$. Thus it suffices to show that any flat of $\beta_{L}$ has to be radial. Argue by contradiction and suppose that there is a flat $F \subset H_{1}(M, \mathbb{R})$ which is not radial. Let $\rho_{1}, \rho_{2}$ be two extremal points in $F$ which are linearly independent and let $\mu_{i} \in \mathcal{M}_{\rho_{i}}(L), i=1,2$. Then there is a cohomology class $c=[\omega]$ such that

$$
F=\rho\left(\mathcal{M}^{\omega}(L)\right)=\left\{h \in H_{1}(M, \mathbb{R}) \mid \alpha_{L}(c)+\beta_{L}(h)=\langle h, c\rangle\right\} .
$$

Since the ergodic components of $\mu_{1}$ and $\mu_{2}$ are also in $\mathcal{M}^{\omega}(L)$, their homologies are also in $F$. Since $\rho_{1}, \rho_{2}$ are extremal points of $F$ and $\rho$ is linear, the homologies of the ergodic components of $\mu_{1}$ and $\mu_{2}$ are respectively $\rho_{1}$ and $\rho_{2}$. In conclusion, we can assume that $\mu_{1}, \mu_{2}$ are ergodic. We need to show that the projection of the orbits in the support of $\mu_{1}$ and $\mu_{2}$ intersect. Since $\mu_{1}, \mu_{2} \in M^{\omega}(L)$ are minimizing measures for the Lagrangian $L-\omega$, the intersection will contradict the Mather's graph property, proving the result.

In the 2-torus $M$, any two integral homology classes in $H_{1}(M, \mathbb{Z})$ which are linearly independent intersect. Let $I: H_{1}(M, \mathbb{Z}) \times H_{1}(M, \mathbb{Z}) \rightarrow \mathbb{R}$ be the intersection form which extends by bilinearity to real homologies. Then, if $r_{2}$ is not a multiple of $r_{1}$ in $H_{1}(M, \mathbb{R})$, we have $I\left[r_{1}, r_{2}\right] \neq 0$. 
We denote by $\pi: T M \rightarrow M$ the canonical projection. For each $i=1,2$, let $\left(x_{i}, v_{i}\right) \in T M$ be a generic point for $\mu_{i}, \Sigma_{i}$ a small transversal segment to $v_{i}$ in $M$ containing $x_{i}, T$ a large return time to $\Sigma_{i}$ of the projected flow of $\left(x_{i}, v_{i}\right)$ so that $\pi\left(\phi_{T}^{L}\left(x_{i}, v_{i}\right)\right) \in \Sigma_{i}$, and $\Gamma_{i}(T)$ a small segment in $\Sigma_{i}$ joining $x_{i}$ to $x_{i}(T)=\pi\left(\phi_{T}^{L}\left(x_{i}, v_{i}\right)\right)$. For each $i=1,2$, we define $C_{i}(T)$ to be the closed curve $C_{i}(T):=\pi\left(\phi_{[0, T]}^{L}\left(x_{i}, v_{i}\right)\right) * \Gamma_{i}(T)$ obtained by concatenating $\pi\left(\phi_{[0, T]}^{L}\left(x_{i}, v_{i}\right)\right)$ with $\Gamma_{i}(T)$. Note that, without loss of generality, we may assume that $\Sigma_{1} \cap \Sigma_{2}=\emptyset$. Choose now two sequences of return times $\left\{T_{k}^{1}\right\}_{k},\left\{T_{l}^{2}\right\}_{l}$ such that $\lim _{k \rightarrow \infty} T_{k}^{1}=\lim _{l \rightarrow \infty} T_{l}^{2}=+\infty$ and $\lim _{k \rightarrow \infty} \operatorname{diam} \Gamma_{i}\left(T_{k}^{1}\right)=\lim _{l \rightarrow \infty} \operatorname{diam} \Gamma_{i}\left(T_{l}^{1}\right)=0$. Since the points $\left(x_{i}, v_{i}\right)$ are generic points for $\mu_{i}$, Birkhoff's Theorem ensures that

$$
\lim _{k \rightarrow \infty} \frac{1}{T_{k}^{1}}\left[C_{1}\left(T_{k}^{1}\right)\right]=\rho_{1} \quad \text { and } \quad \lim _{k \rightarrow \infty} \frac{1}{T_{l}^{2}}\left[C_{2}\left(T_{l}^{2}\right)\right]=\rho_{2} \quad \text { in } H_{1}(M, \mathbb{R}) .
$$

Then by bilinearity of the intersection form, we have

$$
0 \neq I\left[\rho_{1}, \rho_{2}\right]=\lim _{k, l \rightarrow \infty} \frac{1}{T_{k}^{1} T_{l}^{2}} I\left[C_{1}\left(T_{k}^{1}\right), C_{2}\left(T_{l}^{2}\right)\right] .
$$

In order to obtain the contradiction we have to show that there is at least one intersection in $I\left[C_{1}\left(T_{k}^{1}\right), C_{2}\left(T_{l}^{2}\right)\right]$ which is not due to the small closing segments $\Gamma_{1}\left(T_{k}^{i}\right), \Gamma_{2}\left(T_{l}^{i}\right)$.

Note that if $\mu_{1}$ (resp. $\mu_{2}$ ) is supported on a periodic orbit then we can take as $T_{k}^{1}$ (resp. $T_{l}^{2}$ ) a multiple of the period and there is no joining segment $\Gamma_{1}\left(T_{k}^{1}\right)\left(\operatorname{resp} . \Gamma_{2}\left(T_{l}^{2}\right)\right)$. This proves that the intersection occurs when both $\mu_{1}, \mu_{2}$ are supported on periodic orbits, giving the desired contradiction.

For the general case, let $\psi$ be the induced Hamiltonian flow on the projected Aubry set $\mathcal{A}(c)$ in $M$, that is

$$
\psi_{t}(x):=\pi^{*}\left(\phi_{t}^{H}(x, d u(x))\right)
$$

where $u: M \rightarrow \mathbb{R}$ is a critical solution (see Proposition 2.5). We fix $\tau>0$ small enough so that $\psi_{(0, \tau]}\left(\mathcal{A}(c) \cap \Sigma_{1}\right) \cap \Sigma_{1}=\emptyset$, and define $B_{1}\left(T_{k}^{1}\right):=\psi_{[0, \tau]}\left(\mathcal{A}(c) \cap \Gamma_{1}\left(T_{k}^{1}\right)\right)$. Let $\chi_{B_{1}\left(T_{k}^{1}\right)}$ be the characteristic function of $B_{1}\left(T_{k}^{1}\right)$. Since $\chi_{B_{1}\left(T_{k}^{1}\right)} \leq 1$ and the part of $C_{2}\left(T_{l}^{2}\right)$ which may intersect it is contained in $\mathcal{A}(c)$ (recall that, by construction, $\Sigma_{1} \cap \Sigma_{2}=\emptyset$ ), we have

$$
\#\left[C_{2}\left(T_{l}^{2}\right) \cap \Gamma_{1}\left(T_{k}^{1}\right)\right] \leq \frac{1}{\tau} \int_{0}^{T_{l}^{2}}\left(\chi_{B_{1}\left(T_{k}^{1}\right)} \circ \pi\right)\left(\varphi_{s}^{L}\left(x_{2}, v_{2}\right)\right) d s \leq \frac{T_{l}^{2}}{\tau} \quad \forall k, l .
$$

Therefore

$$
\lim \sup _{l \rightarrow \infty} \frac{1}{T_{l}^{2}} \#\left[C_{2}\left(T_{l}^{2}\right) \cap \Gamma_{1}\left(T_{k}^{1}\right)\right] \leq \frac{1}{\tau} \quad \forall k
$$

which implies

$$
\lim _{k, l \rightarrow \infty} \frac{1}{T_{k}^{1} T_{l}^{2}}\left|I\left[\Gamma_{1}\left(T_{k}^{1}\right), C_{2}\left(T_{l}^{2}\right)\right]\right| \leq \lim _{k \rightarrow \infty} \frac{1}{T_{k}^{1}} \frac{1}{\tau}=0 .
$$

Similarly

$$
\lim _{k, l \rightarrow \infty} \frac{1}{T_{k}^{1} T_{l}^{2}}\left|I\left[\Gamma_{2}\left(T_{k}^{2}\right), C_{1}\left(T_{l}^{1}\right)\right]\right|=0,
$$

which proves that projection of the orbits in the support of $\mu_{1}$ and $\mu_{2}$ intersect, a contradiction.

Let $h \in H_{1}(M, \mathbb{R})$ be an irrational homology class, and let $c=[\omega] \in H^{1}(M, \mathbb{R})$ be a cohomology class such that $\alpha_{L}(c)+\beta_{L}(h)=\langle c, h\rangle$. Since $\beta_{L}$ has no flat, the set $\rho\left(\mathcal{M}^{\omega}(L)\right)$ is a singleton (see (4.1)). Let $\Lambda$ be a minimal set in $\tilde{\mathcal{A}}(c)$, and $U \in C^{\infty}(M, \mathbb{R})$ a $C^{2}$-small smooth non-negative function on $M$ such that $U^{-1}(\{0\})=\pi^{*}(\Lambda)$. Then the Aubry set for the 
Lagrangian $L-\omega+U$ is the minimal set $\Lambda$. Moreover $\Lambda$ is not a closed orbit because (the image through the Legendre transform of) any ergodic measure in $\Lambda$ is in $\mathcal{M}^{\omega}(L)$, thus has homology $h$, which is irrational.

The Euler-Lagrange flow of $L$ is the geodesic flow of the metric $g$, which is uniformly hyperbolic (outside $T D^{\prime}$ ). Then the Lagrangian $L-\omega$ has the same flow as $L$. Moreover since the projected Aubry set does not cross $D^{\prime}$ (Lemma 4.1) and $U$ is $C^{2}$-small, the invariant set $\Lambda$ remains hyperbolic with respect to $L-\omega+U$. Hence $\Lambda$ is a non-trivial minimal hyperbolic Aubry set.

\subsection{The case of surfaces of higher genus}

A similar construction can be made in a surface of higher genus as follows. Let $M_{1}$ be a 2-torus, let a hyperbolic metric on $M_{1} \backslash\left\{P_{1}\right\}$ with $P_{1} \in M_{1}, \chi_{1}, \chi_{1}^{\prime}$ two curves surrounding the point $P_{1}$ as above, and let $M_{2} \backslash\left\{P_{2}\right\}$ be a punctured surface of genus $g, g \geq 1$ equipped with a hyperbolic metric. Construct two curves $\chi_{2}$ and $\chi_{2}^{\prime}$ bounding the puncture as in the example above, cut $M_{2}$ through $\chi_{2}^{\prime}$ and join it smoothly to $M_{1}$ along $\chi_{1}^{\prime}$. Then define $M:=M_{1} \# M_{2}$ and consider the smooth geodesic Lagrangian given by (4.2). By construction, $H_{1}(M, \mathbb{R})=$ $H_{1}\left(M_{1}, \mathbb{R}\right) \oplus H_{1}\left(M_{2}, \mathbb{R}\right)$ and the minimizing measures with homologies in $H_{1}\left(M_{1}, \mathbb{R}\right)$ or in $H_{1}\left(M_{2}, \mathbb{R}\right)$ do not cross $\chi_{1}^{\prime}=\chi_{2}^{\prime}$. Also the $\beta$ function satisfies $\beta_{L}=\beta_{L_{1}} \oplus \beta_{L_{2}}$, where $L_{1}$ and $L_{2}$ denote the geodesic Lagrangians obtained as above on $M_{1}$ and $M_{2}$.

Take an irrational homology class $h \in H_{1}(M, \mathbb{R})$ and let $c=[\omega] \in H^{1}(N, \mathbb{R})$ be a cohomology class such that

$$
\beta_{L}(h \oplus 0)+\alpha_{L}(c)=\langle c,(h \oplus 0)\rangle,
$$

where $(h \oplus 0) \in H_{1}(M, \mathbb{R})=H_{1}\left(M_{1}, \mathbb{R}\right) \oplus H_{1}\left(M_{2}, \mathbb{R}\right)$. Let $\Lambda \subset T M$ be the minimal set obtained in Section 4.2, and let $U \in C^{\infty}(M, \mathbb{R})$ be a $C^{2}$-small smooth non-negative function on $M$ such that $U^{-1}(\{0\})=\Lambda$. Then $\Lambda$ is a non-trivial minimal hyperbolic Aubry set for the Lagrangian $L-\omega+U$ on $T M$.

\section{References}

[1] R. Abraham and J. E. Marsden. Foundations of Mechanics. Benjamin, London, 1978.

[2] L. Ambrosio, N. Fusco and D. Pallara. Functions of bounded variation and free discontinuity problems. Oxford Mathematical Monographs. The Clarendon Press, Oxford University Press, New York, 2000.

[3] M.-C. Arnaud. Le "closing lemma" en topologie $C^{1}$. Mém. Soc. Math. Fr., 74, 1998.

[4] M.-C. Arnaud. Fibrés de Green et régularité des graphes $C^{0}$-lagrangiens invariants par un flot de Tonelli. Ann. Henri Poincaré, 9(5):881-926, 2008.

[5] M.-C. Arnaud. The link between the shape of the Aubry-Mather sets and their Lyapunov exponents. Ann. of Math., 174(3):1571-1601, 2011.

[6] M.-C. Arnaud. Green Bundles and Related Topics. In Proceedings of the International Congress of Mathematicians 2010 (ICM 2010).

[7] P. Bernard. Existence of $C^{1,1}$ critical sub-solutions of the Hamilton-Jacobi equation on compact manifolds. Ann. Sci. École Norm. Sup., 40(3):445-452, 2007.

[8] P. Bernard. On the Conley decomposition of Mather sets. Rev. Mat. Iberoam., 26(1):115$132,2010$. 
[9] P. Cannarsa and C. Sinestrari. Semiconcave functions, Hamilton-Jacobi equations, and optimal control. Progress in Nonlinear Differential Equations and their Applications, 58. Birkhäuser Boston Inc., Boston, MA, 2004.

[10] A. Cannas da Silva. Lectures on symplectic geometry. Lecture Notes in Mathematics, Vol. 1764. Springer-Verlag, Berlin, 2001.

[11] F. H. Clarke. Optimization and nonsmooth analysis. Canadian Mathematical Society Series of Monographs and Advances Texts. John Wiley \& Sons, Inc., New York, 1983.

[12] G. Contreras and R. Iturriaga. Convex Hamiltonians without conjugate points. Ergodic Theory Dynam. Systems, 19(4):901-952, 1999.

[13] G. Contreras and G. Paternain. Connecting orbits between static classes for generic lagrangian systems. Topology, 41(4):645-666, 2002.

[14] L. C. Evans and R. F. Gariepy. Measure Theorem and Fine Properties of Functions. Studies in Advanced Mathematics. CRC Press, Boca Raton, FL, 1992.

[15] A. Fathi. Regularity of $C^{1}$ solutions of the Hamilton-Jacobi equation. Ann. Fac. Sci. Toulouse, 12(4):479-516, 2003.

[16] A. Fathi. Weak KAM Theorem and Lagrangian Dynamics. Cambridge University Press, to appear.

[17] A. Fathi, A. Figalli and L. Rifford. On the Hausdorff dimension of the Mather quotient. Comm. Pure Appl. Math., 62(4):445-500, 2009.

[18] A. Fathi and A. Siconolfi. Existence of $C^{1}$ critical subsolutions of the Hamilton-Jacobi equation. Invent. math., 1155:363-388, 2004.

[19] A. Figalli and L. Rifford. Closing Aubry sets I. Preprint, 2011.

[20] A. Figalli and L. Rifford. Closing Aubry sets II. Preprint, 2011.

[21] A. Figalli and L. Rifford. Aubry sets, Hamilton-Jacobi equations, and Mañé Conjecture. Contemp. Math., to appear.

[22] M. Herman. Some open problems in dynamical systems. Proceedings of the International Congress of Mathematicians, Vol. II (Berlin, 1998). em Doc. Math., (Extra Vol. II):797$808,1998$.

[23] A. Katok and B. Hasselblatt. Introduction to the modern theory of dynamical systems. Encyclopedia of Mathematics and its Applications, vol. 54. Cambridge University Press, Cambridge, 1995.

[24] P. Le Calvez. Les ensembles d'Aubry-Mather d'un difféomorphisme conservatif de l'anneau déviant la verticale sont en général hyperboliques. C. R. Acad. Sci. Paris Sér. I Math., 306(1):51-54, 1988.

[25] R. Mañé. Generic properties and problems of minimizing measures of Lagrangian systems. Nonlinearity, 9(2):273-310, 1996.

[26] J. N. Mather. Action minimizing invariant measures for positive definite Lagrangian systems. Math. Z., 207:169-207, 1991.

[27] J. N. Mather. Variational construction of connecting orbits. Ann. Inst. Fourier, 43:1349$1386,1993$. 
[28] I. Nikolaev and E. Zhuzhoma. Flows on 2-dimensional Manifolds. Lecture Notes in Mathematics, vol 1705, Springer-Varlag, Berlin, 1999.

[29] L. Rifford. On viscosity solutions of certain Hamilton-Jacobi equations: Regularity results and generalized Sard's Theorems. Comm. Partial Differential Equations, 33(3):517-559, 2008 .

[30] L. Rifford. Regularity of weak KAM solutions and Mañé's Conjecture. In Séminaire Équations aux dérivées partielles (Polytechnique), to appear.

[31] L. Rifford and R. Ruggiero. Generic properties of closed orbits of Hamiltonian flows from Mañé's viewpoint. Int. Math. Res. Not., 22:5246-5265, 2012.

[32] E. Stein. Harmonic analysis: real-variable methods, orthogonality, and oscillatory integrals. Princeton Mathematical Series, 43. Princeton University Press, Princeton, NJ, 1993.

[33] C. Villani. Optimal transport, old and new. Grundlehren des mathematischen Wissenschaften [Fundamental Principles of Mathematical Sciences], Vol. 338, Springer-Verlag, Berlin-New York, 2009. 